Portland State University

PDXScholar

$1-1-2012$

\title{
A Qualitative Study of Language Beliefs and Linguistic Knowledge in Preservice Teachers Using the Intercultural Communicative Competence Framework
}

Kimberly K. llosvay

Portland State University

Follow this and additional works at: https://pdxscholar.library.pdx.edu/open_access_etds Let us know how access to this document benefits you.

\section{Recommended Citation}

Ilosvay, Kimberly K., "A Qualitative Study of Language Beliefs and Linguistic Knowledge in Preservice Teachers Using the Intercultural Communicative Competence Framework" (2012). Dissertations and Theses. Paper 233.

https://doi.org/10.15760/etd.233

This Dissertation is brought to you for free and open access. It has been accepted for inclusion in Dissertations and Theses by an authorized administrator of PDXScholar. Please contact us if we can make this document more accessible: pdxscholar@pdx.edu. 
A Qualitative Study of Language Beliefs and Linguistic Knowledge in Preservice Teachers Using the Intercultural Communicative Competence Framework

by

Kimberly K. Ilosvay

A dissertation submitted in partial fulfillment of the requirements for the degree of

Doctor of Education

in

Educational Leadership: Curriculum and Instruction

\author{
Dissertation Committee: \\ Susan Lenski, Chair \\ Samuel Henry \\ Dot McElhone \\ Jason Ranker \\ Martha Balshem
}

Portland State University

(C)2012 


\begin{abstract}
Since 1980, the number of people in the United States who speak a language other than English at home has increased by 140\% (United States Census Bureau, 2010). Therefore a greater percentage of students now are multilingual. Throughout the world, multilingualism is considered the norm and monolingualism is the exception (Auer \& Wei, 2008). In the United States, however, policies regarding instruction in schools are still influenced by monolingual ideology that carries expectations and assumptions of assimilation, loss of mother tongues, and defined hierarchical structures. As classroom populations become socially, ethnically, racially, and linguistically more diverse, it is increasingly important for teachers to have an understanding of how to address diversity in schools and for educators to understand how language use and the teachers' role in the classroom impacts learning.

This paper explored the existing language beliefs and linguistic knowledge of preservice teachers as they prepare to enter linguistically and culturally diverse classrooms. The increasing prominence of cross-cultural interactions creates a necessity for teachers to develop intercultural competence. Employing a conceptual framework of intercultural communicative competence theory, this qualitative study investigated experiences and knowledge in linguistics that influence teacher speech acts.
\end{abstract}

Research in fields of applied linguistics such as psycholinguistics, neurolinguistics, sociolinguistics, and educational linguistics revealed basic language knowledge that teachers need before they enter diverse classrooms including knowledge 
of language acquisition, phonology, syntax, semantics, pragmatics, culture, instruction language, and how the brain processes language. The literature from these fields was used to create an instrument that included a demographics questionnaire, beliefs survey, linguistic knowledge assessment, and interview questions. Twenty-three preservice teachers participated in the study to describe their language beliefs and knowledge.

Many of the findings in this study reflected key-findings in the literature; however, this study also found several significant findings that extend existing research. The results revealed significant impacts of 1) individual experiences with culture and linguistic contact, 2) the language used in classrooms, specifically languages other than Standard English and the deep and surface structure of language, 3) linguistic knowledge, specifically phonology, 4) meta-cognitive behavior and reflection, and 5) differences between monolingual and multilingual preservice teachers. The data also indicated that the majority of preservice teachers were concerned about preparedness in teaching in diverse classrooms. Implications for teachers working in culturally and linguistically diverse classrooms and for teacher preparation programs are discussed. 


\section{Dedication}

I would like to dedicate this dissertation to the best father in the world. Without his support, I would not be where I am today. I would not have finished this journey without his love and guidance throughout my lifetime. I would also like to dedicate this paper to my Nana. From the time when I was a young child, she encouraged me to pursue my dream of earning a doctorate. I told her in elementary school that I would someday become Dr. Kim. Now that I have earned the title, I want to thank my father and grandmother. 


\section{Acknowledgments}

I would not have made it through this extensive journey without the amazing support that I have received from family, friends, and even acquaintances. If I were to thank everyone by name, my acknowledgments would be as long as my dissertation. I am a truly blessed person!

I will, however, name a few people who have put up with my many ups and downs over the past five years. My family has been tremendously important to my survival. My parents, husband, and children have helped me juggle many things during the journey. I must thank my eldest son, Robert, because without him, I would not be in the field of education. Anthony, my younger son, was the inspiration for my study of language use. I also want to thank my family for the endless discussions that revolved around my topic.

I must also express my sincere appreciation for my committee. My chair, Dr. Lenski, was always able to find the perspective I could not see and challenge me to think about topics in new ways. Her amazing guidance helped me to fully develop my ideas. Dr. Henry spent much of his time encouraging and supporting my many endeavors relating to learning. He never doubted me, even when I was sure that he was wrong to rely on me. Dr. Ranker helped me to organize my thinking under a conceptual framework. His creative thinking also helped me formulate my thoughts more clearly. Even the new members of my committee were extremely helpful. Dr. McElhone was meticulous and exhaustive in her feedback. Her fresh eyes view and constructive criticism allowed me to continue to see my topic with new points of view. Dr. Balshem was helpful in the feedback of my instrument. Her approach helped me think about 
interview protocols and analysis of my data more systematically. I would have been even more overwhelmed with my data if not for her assistance with organization. As faculty is always busy, I appreciate the time that my committee took to guide me along the way. There are many other faculty members that have encouraged me and taken time out of their busy schedules to assist me.

I want to thank my doctoral cohort and friends. My cohort, as well as other cohorts, was continuously supportive. I appreciate all of the feedback they have given me over the years. My friends must be thanked as well for listening to me work through the process of research and writing. 


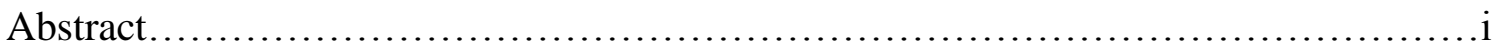

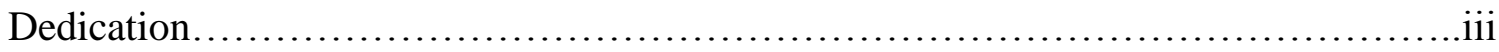

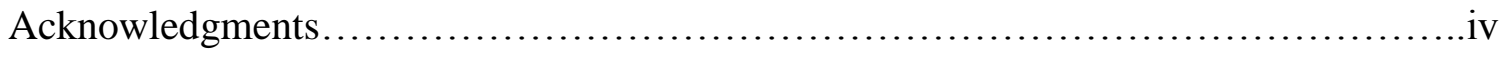

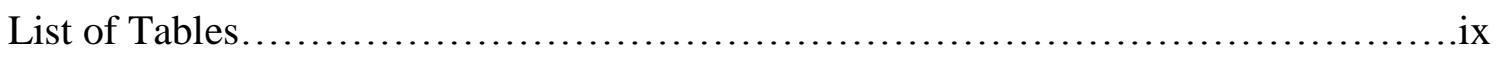

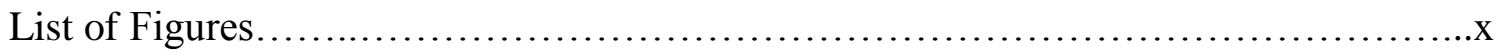

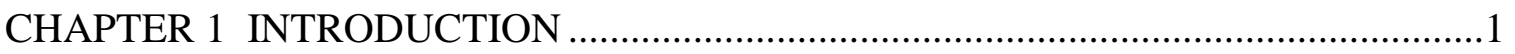

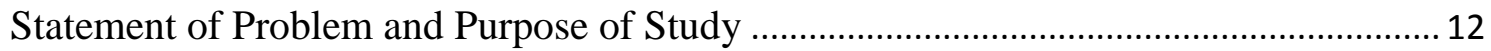

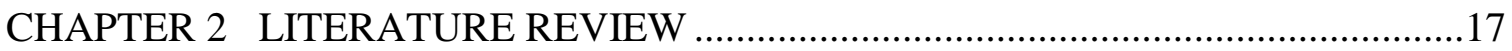

Harmony in Universal Tongues: Intercultural Communicative Competence .................. 17

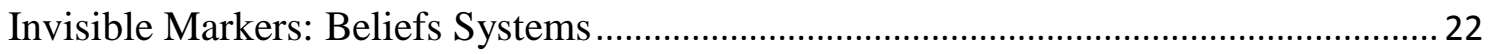

Nothing but the Facts Please: Linguistic Knowledge ................................................... 26

You Will Know a Word by the Company it Keeps: A Psycholinguistic Perspective ..... 36

Language behind the Brow Ridge: A Neurolinguistic Perspective ................................. 43

Language Roots: A Sociolinguistics Perspective …...................................................... 53

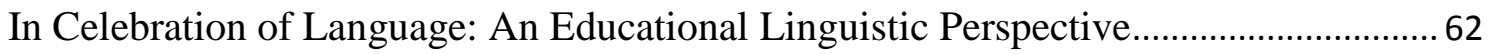

Coursework and Beyond: A Teacher Preparation Program Perspective................73

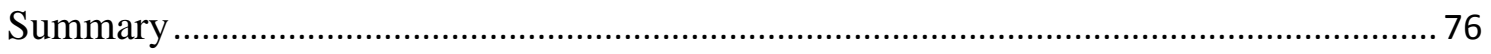

CHAPTER 3 RESEARCH DESIGN AND METHODOLOGY ....................................82

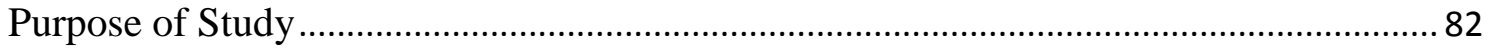

Site Selection and Licensure Program Description...................................................... 84

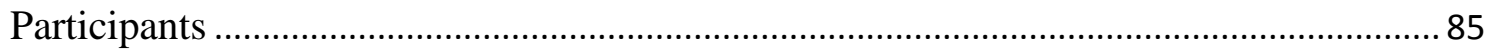




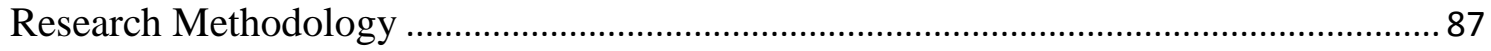

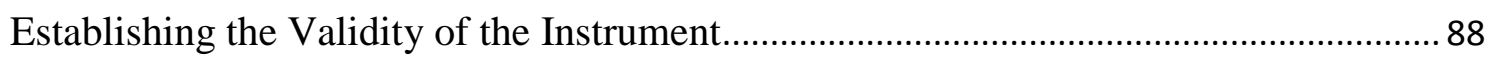

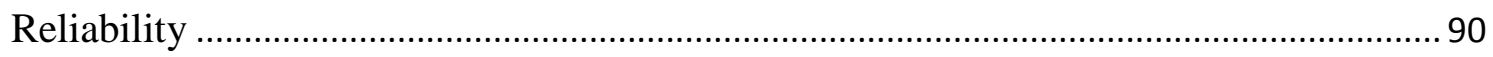

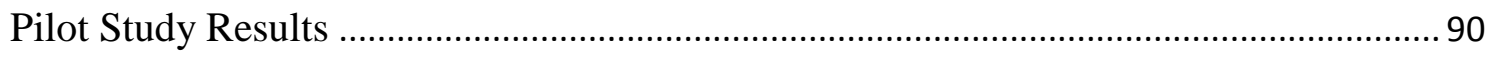

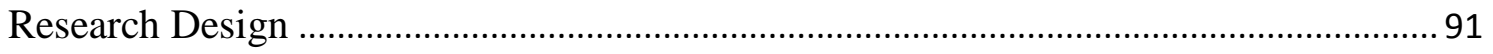

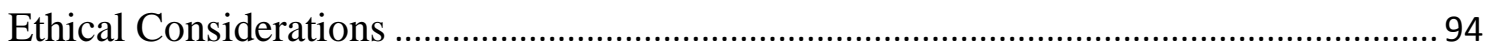

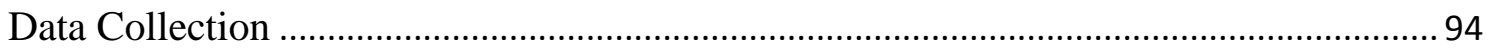

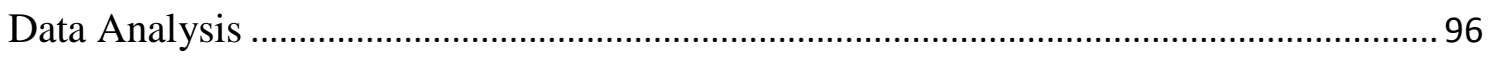

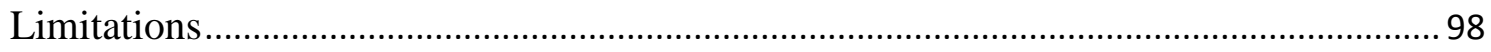

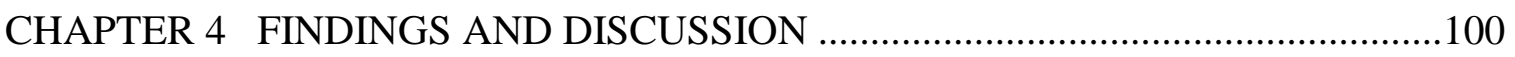

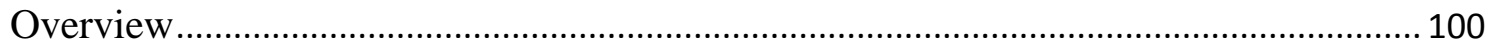

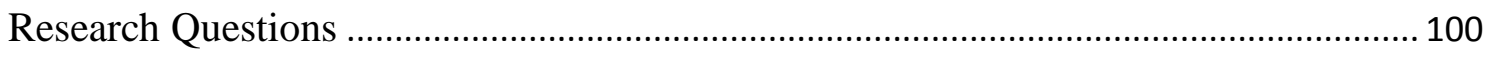

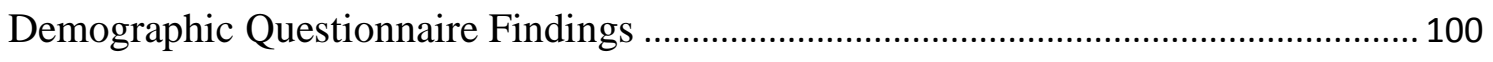

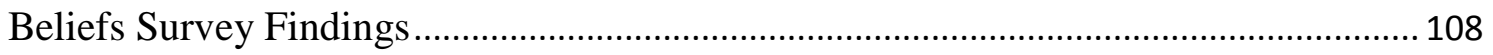

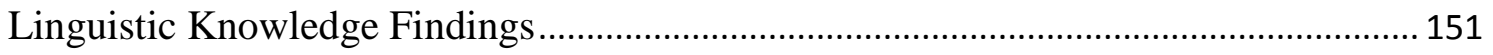

Monolingual and Multilingual Comparison Findings ................................................ 165

CHAPTER 5 IMPLICATIONS AND CONCLUSIONS .........................................184

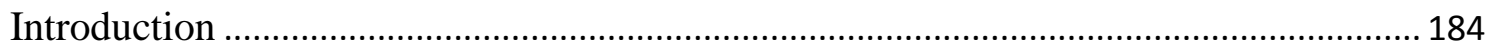

Implications for Teacher Preparation Programs …....................................................... 185

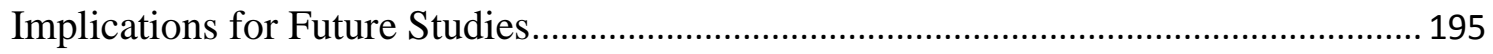

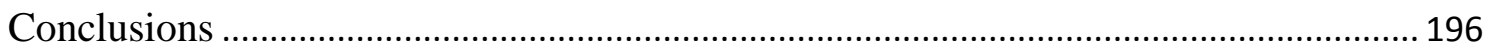

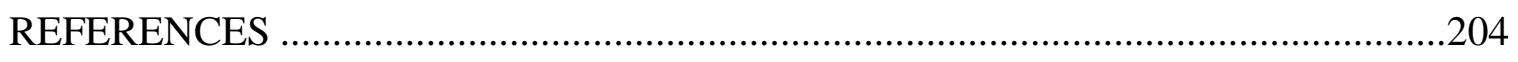

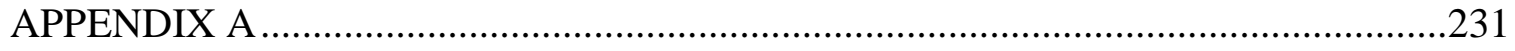

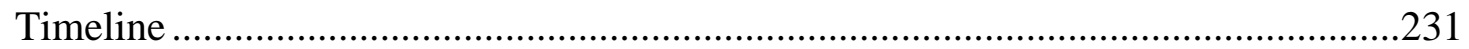

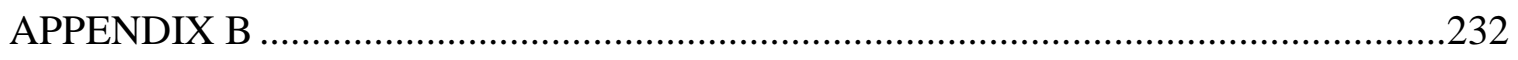




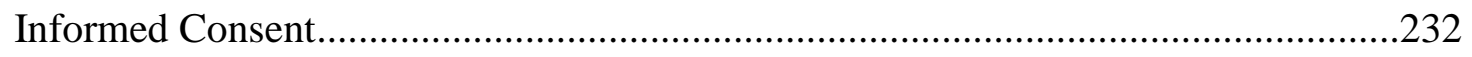

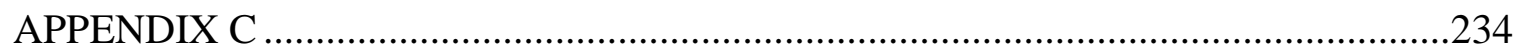

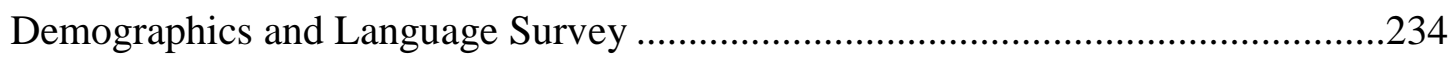

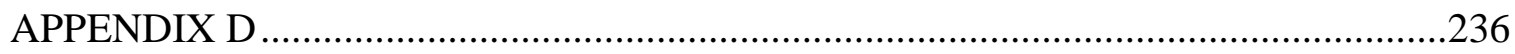

Language Opinion Survey Questions ..........................................................236

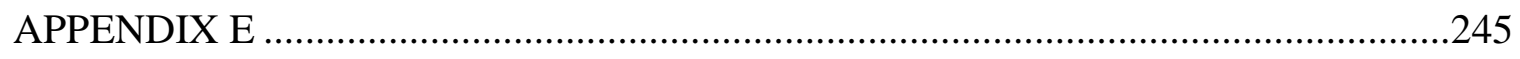

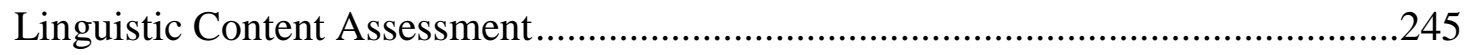

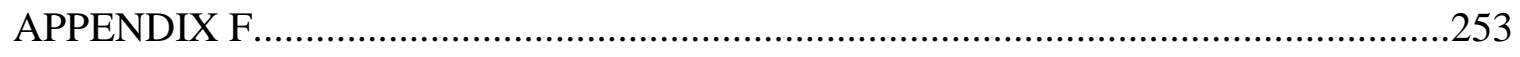

Interview Question Protocol............................................. 253

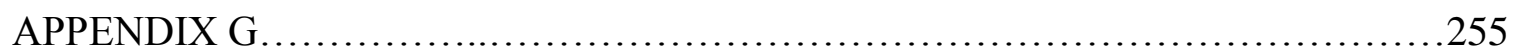

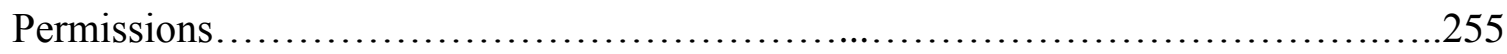


List of Tables

Table 2.1 Types of Knowledge ..................................26

Table 3.1 Qualitative Data Collection Tools..........................95

Table 4.1 Demographic Characteristics of Participants.................105

Table 4.2 Language Acquisition Beliefs ..........................125

Table 4.3 Language System Beliefs ............................. 130

Table 4.4 Communication Beliefs ................................137

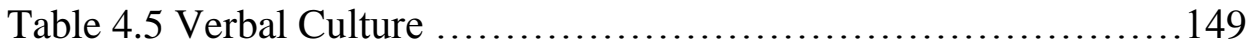

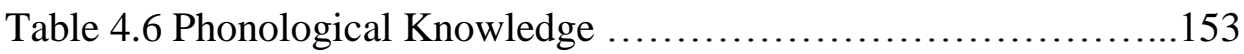

Table 4.7 Grammar Knowledge .................................155

Table 4.8 Semantic Knowledge .................................158

Table 4.9 Pragmatic Knowledge ...............................159

Table 4.10 Cultural Knowledge ..................................161

Table 4.11 Instructional Knowledge ............................163

Table 4.12 Brain Processing Knowledge ...........................164

Table 4.13 Belief Comparisons of Monolingual and Multilingual

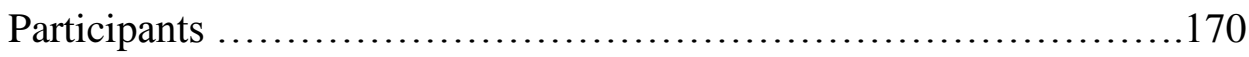

Table 4.14 Linguistic Knowledge Comparisons of Monolingual and

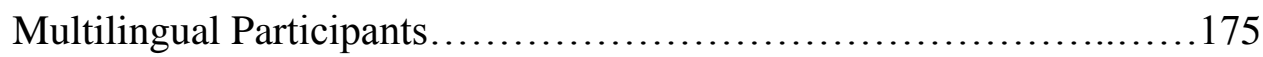


List of Figures

Figure 1 Model of International Communicative Competence ...........22

Figure 2 International Phonetic Alphabet Chart ..........................31

Figure 3 Model of Language Processing in the Brain ...................46

Figure 4 Model of Teacher Language Awareness.........................74 


\section{CHAPTER 1}

\section{INTRODUCTION}

Throughout the world, multilingualism is considered the norm and monolingualism is the exception (Auer \& Wei, 2008). In the United States, however, policies regarding instruction in schools are still influenced by monolingual ideology that carries expectations and assumptions of assimilation, loss of mother tongues, patriotism, and defined hierarchical structures. Standard English was developed, in part, so that it could unify shared experiences while maintaining monolingualism (Justice, 2004). According to Spolsky and Shohamy (1999), policies that require "English only" in United States' schools create authoritarian environments in which "one language is recognized and associated with the national identity; others are marginalized" (p. 96). Furthermore, Kono (2001) suggested that there is pervasive resistance to pluralism in United States classrooms, where teacher behavior is guided largely by monolingual ideals. Within a post-9/11 context, the United States has sought to revive the values, philosophies, customs, and practices of a monolingual society as evidence of patriotism, forgoing the advantages of bilingualism and biculturalism (Wiley, 2007).

As classroom populations become socially, ethnically, racially, and linguistically more diverse, it is increasingly important for teachers to have an understanding of how to address diversity in schools. Since 1980, the numbers of people in the United States who speak a language other than English at home has increased by $140 \%$ (United States Census Bureau, 2010). The Hispanic/Latino population in the United States alone rose from 35.5 million in 2000, to 50.5 million in 2010 (United States Census Bureau, 2010). 
Given the increasing number of multilingual students in the United States, it is important for educators to understand how language use and the teachers' role in the classroom impacts learning.

\section{Benefits of Diverse Language Exposure and Use}

According to behaviorists and neurolinguists, prolonged diverse language exposure and speaking multiple languages have been found to increase activation of language areas and phonetic processing in the brain, which enhances abilities such as social and cognitive skills; and oral, reading, and writing skills (Baker, Kovelman, Bialystok \& Petitto, 2003; Norton, Baker \& Petitto, 2003). Nieto and Bode (2008) explained that despite negative perceptions of bilingualism, there are advantages to hearing and knowing multiple languages at both ends of the life span. Kovacs and Mehler (2009) found that bilingual infants are more precocious decision makers with stronger cognitive control. Nieto and Bode (2008) also described neurological studies that demonstrate that bilingual brains stay sharper longer and develop symptoms of aphasia later in life than monolingual brains. Similarly, studies of multilingual populations indicate there are many cognitive, social, and professional advantages to being multilingual (Bialystok, Craik, \& Ruocco, 2006). Speaking multiple languages also encourages speakers to reflect on the meaning, value, and utility of their first languages. Furthermore, multilingual students have a relatively enhanced ability to generate novel thoughts and word associations because they operate with multiple language systems and a larger mental lexicon (Dijkstra, 2007).

\section{Language Acquisition}


In a natural language learning process, the speakers of a language, even the very young, are capable of understanding any utterance spoken in a language and of producing utterances that can be accepted as meaningful by other speakers (Pinker, 2007). When exposed to multiple languages early in life, the recognizable utterances increase. Most people begin learning the complexities of their first language even before birth and continue to learn into adulthood (Halliday, 1975). According to the "continuity" hypothesis, language processes are similar during development and adulthood in principle (Friederici, 2005). Further, the similarities between brain response patterns observed in children and adults support the view that language develops in a continuous manner. According to Chomsky (1968), Freeman and Freeman (2004), and Pinker (2007), children with normal intellectual capacity acquire the first language with relatively little exposure and virtually no specific training in the intricate structure of specific rules and principles. However, with second language acquisition,

Learning another language takes you into the less easily charted territory of learning a new set of grammatical principles: new tenses, new ways to think about time and the physical world, new ways to organize words into sentences, and new idioms and expressions (Ottenheimer, 2009, p.44).

Generally speaking, there are two opposing philosophies of language acquisition and comprehension that are important to consider when exploring language use: functional and generative linguistic approaches. Functional linguistic theories downplay the importance of biology and stress the overt structure of language and instruction. Krashen (1982) explains that "learning" occurs when the predominant mode of increasing knowledge about a language is explicit instruction as opposed to innate acquisition of a 
language. Stressing innate acquisition, generative linguistic theories posit that immersing students in language-rich activities and providing opportunities for the practice of both speech and written communication will stimulate natural language development; this process is termed "acquiring" (Gee, 2001). Teachers belonging exclusively to one or the other of these philosophical camps will likely have differences in opinion regarding the form and function of language and language acquisition; they may also differ as to the degree to which biology influences the acquisition of syntax, semantics, semiotic structure; and other attributes of language. Consequently, different attitudes toward acquisition yield different approaches and different instructional practices in the classroom (Freeman \& Freeman, 2004).

It is often difficult for teachers and researchers to study the language-learning processes children use to acquire and comprehend language. Instructional sequences are often in direct conflict with natural ways of learning (Allington, 1984). "What we observe, then, is not a child's learning processes but their responses to instructional demands" (Lindfors, 1991, p. 243). Because language processing is sometimes only indirectly observable, teachers must have reasonably thorough comprehension of language and be skilled in the analysis of language issues.

\section{Language Use and Usage}

Language is used as a tool to share experiences, needs, desires, thoughts, and ideas. Lindfors (1991) expresses the benefits of teachers using language to facilitate social, cognitive, and analytical communication. Agar (1994) further expresses the benefits of using language to create shared meanings with diverse student populations. 
Knowing how language serves as a communication device that uses rules of syntax, semantics, and pragmatics and serves to mark class, culture, and identity helps teachers become aware of language differences.

Ruiz (1984) suggested reorienting classroom environments to reflect a languageas-a-resource model. This model is based on research by Bialystok (2007) and others that found benefits in knowledge and exposure to more than one language. Many teachers, however, lack the desire or ability to establish classroom environments that allow for acceptance of linguistic diversity (Fillmore \& Snow, 2000). Lippi-Green (1997) contends that all spoken languages are equally capable of expressing meaning with a full range of ideas and experiences and developing new vocabularies and ways to meet the new needs of its users. However, individual language development and personal language preference is complex. "Spoken language varies for every speaker in terms of speech sounds, sound patterns, word and sentence structure, intonation, and meaning, from utterance to utterance" (Lippi-Green, 1997, p. 25). Despite research that suggests the importance of acknowledging a students' linguistic background in the classroom, teachers express concern about the difficulty in establishing an effective linguistic environment suited to all learners. A teacher's linguistic knowledge, however, provides options for addressing linguistic differences instead of discounting these differences.

With an informed knowledge of language use and usage, teachers have the ability choose appropriate language and to teach basic proficiency in language. The effectiveness of language hinges on language competence. In order to help students gain language competence, teachers must themselves be knowledgeable about language 
systems (Scollon \& Scollon, 2001). Competency in communication involves "listening to viewpoints different from one's own, summarizing them in ways others can recognize, comparing and contrasting positions, spotting contradictions and non sequiturs, and coming to conclusions that contribute to a continuing conversation of ideas" (Graff, 2003, p. 23).

Teachers need an understanding of how to carefully choose their own language to allow for student comprehension, as well as knowledge about how to help students learn negotiating language use with others. Earlier studies of teacher/student discourse focused primarily on the syntax of students' speech (Larson-Freeman, 1985). Hatch and WagnerGaugh (1975) were among the first to recognize that to gauge the effect of input on student learning, researchers needed to look at the sources of input as a whole, not focusing solely on one aspect. Furhtermore, Chaudron (1985) made a distinction between input and intake, pointing out that presenting a certain linguistic form to a learner does not necessarily qualify it as input, because input is not necessarily "what goes in," but is rather "what is available for going in." Though it is the learner who controls this input (or more properly his or her intake), it remains an important concept for teachers as they select mutually intelligible classroom language.

\section{Proficient and Competent Communication}

An awareness of effective classroom communication is critical in order to allow teachers to determine their roles in students' language development. Proficiency in communication means different things to different speakers, depending on the context in 
which it is being assessed (Ottenheimer, 2009). A student may have understood on one level what a teacher has said, but on another level they perceived teacher talk completely differently than the teacher intended. For example, some students display proficiency on language tests, but their semantic knowledge does not correspond to their teacher's expectations. Also, a student may have understood the vocabulary spoken, but may not have understood the context or relevance of the sentence. A teacher's evaluation of student proficiency must take into consideration many aspects of language use.

According to Chomsky (1968), "linguistic competence" is the key to language proficiency. According to Hymes (1972), however, "communicative competence" is the core measure of language proficiency. Linguistic competence requires producing and recognizing grammatically correct expressions in language. Communicative competence relies on speaking appropriately in a variety of social situations and is more than correct syntax. The perspective of a grammarian - who thinks of language as a system and the perspective of a linguist, who views language as a less strictly defined means of communication - may yield different interpretations of language proficiency (Kachru, 2008).

Another type of competence that is important in classrooms today is intercultural communicative competence. According to Samovar, Porter, and McDaniel (2009), intercultural communicative competence means having cross-cultural and cross-linguistic awareness, negotiation of language, and conflict resolution typically among peoples of different backgrounds. Today, there are more immigrants and people born in the United States who choose to retain their cultural and linguistic heritages by living near others 
who share their experiences than ever before; this phenomenon contrasts with the linguistic practices of previous generations that chose to or were forced to assimilate with the dominant culture of the United States (Paris \& Ball, 2009). This new pattern has created a greater need for intercultural communicative competence within the classroom environment.

\section{Language Use and Methods of Instruction}

The diverse population present in classrooms requires diverse language use during instruction. Progressivists believe that children acquire their native languages by being immersed in rich, meaningful, and natural communicative settings (Gee, 2001). Nieto and Bode (2008) further explained that to support multilingual students, teachers must understand how students learn subsequent languages and have knowledge of multiple instructional models. Analyzing what this means at different levels of language development within monolingual or multilingual communities is important to understand language acquisition and instruction. Scholars have found that for native language speakers (L1) beginning instruction with simplified speech allows access to language learning, followed by increasingly complex grammar and vocabulary in the later stages of development which advances monolingual learners more rapidly (Hoff, 2006; Snow, 1972). Corresponding results, however, have not been found for people learning second languages (L2). Ellis (1999) found mixed results when reviewing research on language instruction using simplified input versus complex language. L2 research has not demonstrated a correlation between a particular method or type of instruction and a concomitant increase in the proficiency of language learning (Krashen, 1982). Neither simplified speech nor continuous exposure to complex language methodologies within 
the classroom addresses the fact that a child's individual learning strategies and their native language are also important in mastering behavior associated with the use of language. Therefore, teachers must be able to choose from various methods of instruction in order to find effective methods for each student.

Furthermore, subscribing to one philosophy such as functional or generative linguistics or dichotomies such as "immersion in practice" or "explicit instruction" over the other may not be effective for use in culturally and linguistically diverse classrooms. Additionally, much of the instructional material used in classroom teaching - such as teacher guides, student texts, workbooks, and so on - is highly influenced by the ideological underpinnings of monolingual standard language use (Jenkins, 2009). In addition, Heath (1986) suggests that exportable models of curriculum or instruction that are not designed to be adapted should not be utilized by teachers. Heath also recommends the exclusion of methodologies and curricula that are based on the assumption that the path of language development is the same for all children. Instructional methods should differ in style and genre as well as explicitly focus on the structure of oral language that includes sounds, morphology, syntax, and semantics (Freeman \& Freeman, 2004; Gee, 2001; Harste, Woodward, \& Burke, 1984).

Another complicating factor in choosing an instructional method that is adaptable to diverse classrooms is that students also exhibit culturally different patterns of language socialization (Ovando, 1997). Researchers have documented "the extent to which students possess different kinds of minds, and therefore learn, remember, perform, and understand in different ways" (Gardner, 1991, p. 11). Because of the various learning 
styles of students from different linguistic backgrounds, the methods necessary for teaching students must also vary. Teacher beliefs powerfully impact their instructional style (Cess-Newsome, 2002; Ernst, 1989).

\section{Teacher Belief Systems}

Barcelos (2003) explains that a teacher's beliefs determine which method or methods of instruction are used in the classroom. Also, a teacher's belief system about language and knowledge and beliefs regarding how to analyze language issues in the classroom guide language behavior. Beliefs have been said to be the best predictor of an individual's behavior and also influence the teacher's perceptions and judgments (Brown, 2004; Hudson, 2010; Pajares, 1992). Understanding the nature of beliefs is essential to understanding an educator's choices, decisions, and effectiveness regarding issues of diversity. Beliefs and knowledge about language also play a major role in how educators respond to and understand students of different linguistic and cultural backgrounds (Furman, 1998). There are researchers and practitioners who feel there is a growing perception that some models of belief systems have become overly simplistic (Woods, 2003).

Woods (2003) further explained that effective models and findings revolve around three approaches that are said to differ from popular views. The first approach is that beliefs are not isolated individual ideas, as often suggested by the research, but are interconnected and structured (Barcelos, 2003). The second is that beliefs are not constant entities within the individual, but are situated in social contexts and formed through specific instances of social interactions and, as a result, are constantly evolving 
(Routman, 2005). The third is that "beliefs are not separable or separate from other aspects of learners' cognitive processes, but integrated in a larger dynamic model of thought and action, forming not the periphery but the central framework" within which all teaching and learning take place (Woods, 2003, p. 202).

It is through reflection that belief systems can be revised or strengthened (Routman, 2005). The underlying structures of many United States school systems are guided by the perception that students who are not proficient in English have language problems that must be overcome (Freeman \& Freeman, 2004). Teachers within these school systems who believe in "language as a deficit" orientations perpetuate "subordinate status" for languages other than English. Edl, Jones, and Estell (2008) demonstrated that a student's language proficiency influenced a teacher's view of whether a particular student was a low or high academic achiever. More specifically, they found that teachers rated Latinos who were dual language learners as lower academic achievers than Latinos that were proficient in English. Also, a teacher's knowledge of the articulation, psychological, neurological, sociological, and educational aspects of being multilingual creates belief systems that enable them to view language as a resource for the classroom. Thinking of multilingual students as a resource means understanding the multifaceted lives of linguistically heterogeneous groups of students.

Beliefs about language as a resource can be viewed within a model of interconnectedness that allows beliefs to constantly be revised as new knowledge is gained and new experiences occur. New awarenesses form belief systems situated in social interactive contexts with integration from various aspects of the cognitive process 
evolving our thoughts and actions (Woods, 1997). Knowledge is how things are (the presumption of truth), but beliefs are a value judgment on how things are (Woods, 1997). Beliefs and knowledge have an integral relationship.

\section{Statement of Problem and Purpose of Study}

For the purpose of this study, the term multilingual was used to describe people who speak or know more than one language. The increase in the number of multilingual students in classrooms has important implications for the educational system in the United States. These implications have a profound impact on the teachers who are in direct contact with diverse student populations. Teachers must understand various discourse activities and possible miscommunication issues, which will require specific language training in teacher-preparation programs. These understandings begin with metalinguistic awareness that shapes personal belief systems.

One implication for the educational system is that teachers face a wide variety of language needs in their student populations. To facilitate comprehension, teachers must be cognizant of the features of language and the effects of language use (Hudson, 2010). Effective communication requires eight major structural components (Samovar, Porter, \& McDaniel, 2009). To manage oral messages and create meaning, speakers must undergo negotiation of the 1) sender, 2) message, 3) channel, 4) receiver, 5) response, 6) feedback, 7) environment, and 8) the noise encountered in conversation. As teachers gain linguistic knowledge, the ability to understand students' linguistic needs increases.

Teachers also must consider potential miscommunication issues, such as pronunciation differences (Underhill, 1994), lack of comprehension due to deficiencies in vocabulary learning and lexical density (Ellis, 1999), language ambiguity (Chaundron, 
1982), sentence structure differences, teacher/student background differences (Rao, 2005), differences in individual learning styles (Gardner, 1991; Nagy, 1993), interactional factors that inhibit oral input (Ellis, 1999), and cultural interpretation variances (Lippi-Green, 1997). Studies have also shown that multilingual students' language- processing systems organize and use language differently than do monolingual students (Dijkstra, 2009). Students typically encounter academic Standard English speech in United States schools, but they use other languages, dialects, and registers elsewhere resulting in frequent communication misunderstandings.

Another implication for the educational system is the lack of preparation teachers receive about instructing linguistically diverse students. Studies indicate that teachers without specific training in linguistics tend to underestimate or overlook the linguistic knowledge of their students (Godley, Sweetland, Wheeler, Minnici, \& Carpenter, 2006). Teacher education programs that include linguistic information in the curriculum - such as the articulation and neural language process, prosody variants, and language variation in deep and surface structure - produce teachers who create and maintain respectful environments, appropriate teacher talk, and less prescriptive attitudes toward language use (Freeman \& Freeman, 2004; Godley, Sweetland, Wheeler, Minnici, \& Carpenter, 2006; Justice, 2004). Providing relevancy to linguistic course content is the key component in developing curriculum. Additionally, linguistic curriculum embedded in teacher preparation programs that improved a teachers' understanding of language skills, not only in speaking but also in reading, writing, and listening, create positive learning environments (Hudson, 2010). 
Preservice teachers may not explicitly consider the reason behind their language choice and impact of their language use or their student's linguistic diversity (Ball, 2000). Bernstein Ratner, Berko Gleason, and Narasimhan (1998) stated that "Our talent for speaking well and understanding competently are aspects of our linguistic knowledge, whereas our ability to reflect upon our language - our understanding of how we do these things - represents an aspect of our metalinguistic knowledge” (p. 29). By developing metalinguistic knowledge, teachers can evaluate the variants in their own language use as well as negotiate meaning with their students (Freeman \& Freeman, 2004; Wilson, 2001). When communication begins to span cultural and linguistic discourse systems, speakers and listeners become hyperconscious of communication, and this tends to produce metalinguistic knowledge (Scollon \& Scollon, 2001). Metalinguistic knowledge is important when addressing issues in intercultural communication. Teachers exhibit more awareness of code switching and develop more positive beliefs toward language diversity during reflection of their own metalinguistic knowledge (Hudson, 2010).

Linguistic research has explored teacher language beliefs by focusing explicitly at only one area of study at a time such as phonology (Snedeker \& Trueswell, 2004) or culture (Nocon \& Cole, 2009). Linguistic research has also examined relationships between fields of study such as neurobiology and linguistics (Ullman, 2008), or psychology and language (Woods, 2003). Ovando (1997) points out that the studies of language through different points of view, such as at the physical level or the cognitive point of view, have added to research literature in a positive way. However, from a pedagogical point of view, these different ways of studying language must be integrated in order to truly understand the process of learning $a$ language and learning through 
language. Additionally, no published studies have examined the relationship between knowledge of articulation, psycholinguistics, neurolinguistics, sociolinguistics, and educational linguistics and how these relationships may manifest in language use in the classroom. Furthermore, no studies have explored the relationship between the beliefs preservice teachers have about issues in these fields and the knowledge they carry about linguistics and how their beliefs and knowledge apply to the classroom.

This study explores preservice teachers' beliefs and knowledge about language. The study specifically investigates the language beliefs and knowledge preservice teachers have as informed by research findings in the fields of applied linguistics such as the sounds of language, psycholinguistics, neurolinguistics, sociolinguistics, and educational linguistics, and through the framework of intercultural communicative competence (ICC) (Gee, 2001; Halliday, 1978; Walenski \& Ullman, 2005). Language is embodied in the brain; therefore, knowledge of how the brain processes and stores language (neurolinguistics) is pertinent to a teacher's foundational background knowledge. Psycholinguistics deals with the study of the mental process that underlies comprehension and speech production. This knowledge is important not only for understanding a student's language acquisition but also for understanding the teacher's own speech production in the classroom. Sociolinguistic research involves understanding a variety of language systems, linguistic structures, and language patterns used in contextualized social settings. The relation between language and culture plays a key role in classrooms. Teaching multilingual students means understanding the multifaceted lives of linguistically heterogeneous students. Boyer (1990) explained that using the ICC framework provides an understanding of the integration of information. He further states 
that ICC allows the making of connections across disciplines and placing specialties in a larger context.

Given that a teacher's pedagogical beliefs and epistemological knowledge about language (Freeman \& Freeman, 1994) critically influence students' outcomes, it is essential to explore these systems to understand how these factors drive outcomes. The primary purpose of this study is to describe teachers' beliefs and knowledge about language. The description of the preservice teachers will be enriched by analyzing beliefs and knowledge from the perspectives of articulation, psychology, neurology, sociology, and education. This interdisciplinary approach reveals a more holistic picture of belief systems that influence language interactions in a multilingual classroom. The following questions guided this investigation:

1) What language beliefs do preservice teachers have at the beginning of a teacher preparation program?

2) What linguistic knowledge do preservice teacher have at the beginning of a teacher preparation program?

3) What are the similarities and differences in the beliefs and knowledge about language between monolingual and multilingual preservice teachers? 


\section{CHAPTER 2}

\section{REVIEW OF LITERATURE}

\section{Harmony in Universal Tongues: Intercultural Communicative Competence}

With increased global interdependence, producing intercultural communicative competent (ICC) citizens should be an educational priority (U.S. Department of Education, 2011). Few higher educational institutions, however, have focused on preparing teachers in the development of intercultural competence (Deardorff, 2006). If teachers lack intercultural competence, how can they be sensitive to the diversity present in their classrooms?

Not unlike the diversity exhibited throughout the world, the diversity in classrooms signals a call for a global mindset that includes intercultural competence. Within the perspective of a global mindset, one exhibits curiosities about the world, acceptance of diversity, and attributes that enable cooperation between socio/cultural systems (Samovar, Porter, \& McDaniel, 2009). In addition, Noddings (2005) suggests that the classroom perspective must represent "ways of life," not just the way of the wealthy nation (p. 2). She also argues that though many believe that a global mindset is in opposition to patriotism of one country, the need for patriotism solely to one country is no longer a relevant necessity. Moreover, patriotism shown to one world with people who cooperate and appreciate each other is of greater importance.

Global interdependence and cross-cultural encounters are reshaping the world. When diversity and global concerns are strategically linked to teaching and learning outcomes, the development of intercultural communicative competence (ICC) leads to enriched perspectives, greater understandings of ambiguities, and skills critical for 
success (Scollon \& Scollon, 2001). Language use is impacted by the assumptions speakers and listeners have about culture. These assumptions often create misunderstandings. To develop ICC, however, it is necessary to have a solid understanding of ambiguities, interpretations, and inferences in language.

Language is fundamentally ambiguous because interpretation is based on experience (Pinker, 2007). Yet, communication is effective when students and teachers share assumptions and knowledge about the world (Scollon \& Scollon, 2001). Although communication between people who are members of the same cultural or linguistic group can result in misunderstandings, when members of linguistically diverse groups communicate, there is a greater likelihood of misunderstandings. These misunderstandings can occur for many reasons and may result from differences in discourse patterns, which include differences in pronunciation, vocabulary, grammar usage, experience, language processing, prosody, and cultural interpretations. Scollon and Scollon (2001) maintained that to resolve miscommunication issues, it is necessary to have knowledge of both cultural and linguistic systems.

Knowledge of these language systems allows language users to develop strategies to negotiate ambiguities. Yet, all speakers interpret utterances differently based on varying assumptions held by members of different groups. Interpretive understandings (or misunderstandings) are often a source of confusion in classrooms. Effective communication requires that teachers understand that different discourse systems carry different expectations and inferences based on different world views. Furthermore, teachers need to recognize their own limited understandings of different discourse systems (Scollon \& Scollon, 2001). 


\section{Defining ICC to Achieve Competence}

Deardorff (2006) contended that ICC must be defined to establish criteria for measurement. She further emphasized that for ICC there has been general agreement on measurement components such as context, situation, and relation; however, scholars have no consensus on a definition of communication or competence, especially when it deals with cross-cultural or cross-linguistic understandings. Chen (1993) emphasized harmony in relationships using respectful human behavior and communication in his broad definition of ICC. Bryam (1997) defined ICC by including (a) knowledge of others, knowledge of self, (b) skills to interpret and relate, (c) skills to discover and or interact, valuing others values, beliefs, and behaviors, (d) knowledge of linguistics, and (e) relativizing oneself. Further, King and Baxter-Magolda (2005) contend that application of this knowledge is often omitted from language use. They argue that these beliefs and knowledge should motivate action. According to Barcelos (2006), scholars most widely accept a definition of ICC that includes the negotiation of language between speakers of different native languages resulting in some form of meaning for all speakers. In her exploration of an appropriate definition, Deardorff (2006), however, identified specific components of ICC including (a) world knowledge, (b) language proficiency, (c) cultural empathy, (d) approval of people and cultures, and (e) ability to practice ones profession in an intercultural setting. She also contended that many definitions of intercultural communicative competence reflect Western - centric ideologies of individuals in their perspectives.

In analyzing the various definitions for ICC, I developed a definition that reflects basic components of ICC that experts agreed upon and can be incorporated into diverse 
classrooms in the United States. The definition used for this study also specifically aligns with the purpose of the study which is to explore language beliefs and knowledge of preservice teachers. Therefore, I will use the following definition of ICC for this study: ICC means having the ability to use and negotiate language between people of differing native languages in a way that expresses respect for other cultural and linguistic systems to convey and receive meaning with an understanding that everyone has a different world view that influences their language use and perception. The underlying characteristics that show competence are one's awareness of personal values, beliefs, and knowledge; one's degree of openness to understanding ideas, values, norms, and behaviors different from one's own; and one's capacity to create different behavioral tendencies to express appreciation of others cultural and linguistic resources.

\section{Developmental Stages of ICC}

The definition of ICC does not take into account the degrees of competence that people possess (Deardorff, 2006). To address the developmental issue, the American Council on International Intercultural Education (1996) created a model for the development stages of competence. Based on this model, individuals proceed through developmental stages: (a) recognition of global systems and their interconnectedness, (b) intercultural skills and experiences, (c) general knowledge of history and world events, and (d) detailed areas of specialization. These developmental stages have no particular order; the process is a continuous revision of beliefs when new experiences and acquisition of knowledge are obtained. 
Furthermore, Triandis (2009) believes that there are four stages of communicative awareness affirming competence. Unconscious incompetence comes about when a person comes into contact with members from other cultures and is oblivious of miscommunication because of the belief that everyone is more or less like him or her. Conscious incompetence is when a person realizes there are communication issues, but does not know exactly why. Conscious competence is a stage in which some knowledge of the other culture exists, but communicating in a different way takes concerted effort. Finally, unconscious competence occurs when communication with others is effortless and appropriate. Essential elements of ICC stem from personal beliefs and knowledge about language and culture that shape personal behavior (Deardorff, 2006).

\section{Model of Intercultural Communicative Competence}

The model of ICC that is used to frame this study is based on the work of Deardorff (2006) and others and takes into account the developmental nature of ICC (see Figure 1). I developed the model to emphasize the importance of individual foundational belief systems. The model depicts the relationship between the beliefs about the values of different cultures, the knowledge that provides awareness, the attitudes of respect, and the resulting behavior as a process that an individual continuously refines. This model of ICC shows how participants make changes to beliefs, attitudes, or behavior as new knowledge and skills are acquired. 


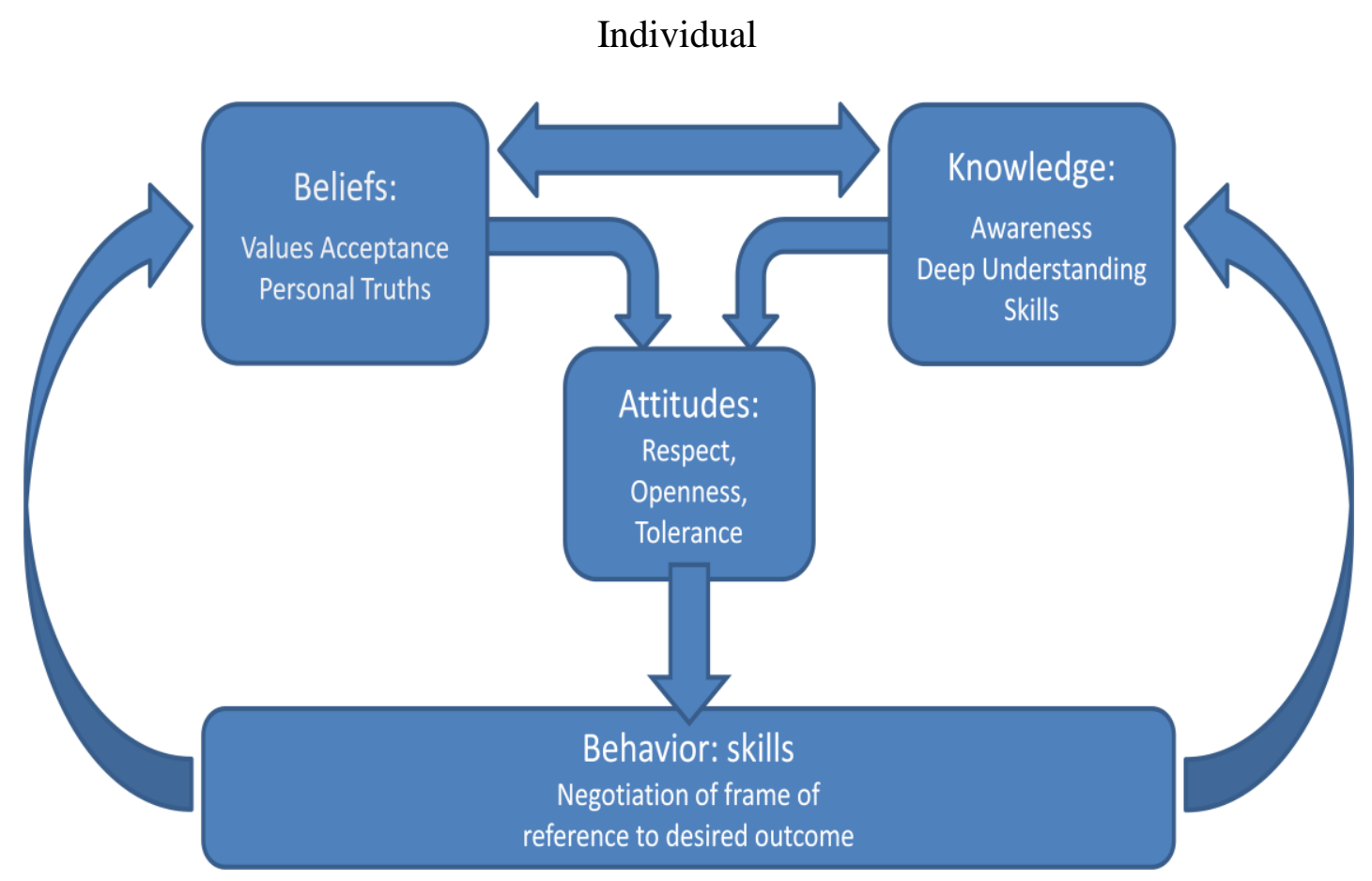

Figure 1. Model of intercultural communicative competence (Ilosvay, 2012)

\section{Invisible Markers: Belief Systems}

One predominant component of intercultural communicative competence is the individual's belief system. Barcelos (2006) explained that beliefs have both a cognitive dimension (i.e., linguistic knowledge) and a social dimension (i.e., interactions with others and environment). In the cognitive dimension, teachers rely on their knowledge of various language systems to create expectations of success for their students. In the social dimension, a view of the language use in a classroom includes the teacher's perspective and the student's perspective. According to Bakhtin (1981), language that is 
dialogic in nature confirms the reciprocal nature of language use in the social dimension. Bakhtin states:

Everything means, is understood, as a part of a greater whole - there is a constant interaction between meanings, all of which have the potential of conditioning others. Which will affect the other, how it will do so and in what degree is what is actually settled at the moment of the utterance. (p. 426)

\section{The Brain's Involvement in Belief Systems}

Both the cognitive and the social dimensions of belief affect the capacity of the mind to believe or disbelieve linguistic propositions and to control behavior (Harris, Sheth, \& Cohen, 2008). Recent neurological studies reveal the differences in areas of the brain involved in belief, disbelief, and uncertainty (Harris, et al, 2008). They found that agreement with statements of belief activated areas of the prefrontal cortex (VMPFC), predominantly the left hemisphere where emotional associations occur. Alternatively, brain areas engaged in dissent were in the dorsal structures commonly associated with executive functioning and decision making. In addition, disbelief caused more areas of the brain to activate and release negative signals. Judgments of uncertainty were associated with areas in both the left and right hemispheres and with a positive signal change. When uncertainty or disbelief are present, more thought processes seem to occur. Moreover, critical thinking skills develop when the brain is engaged in actively making meaning and establishing relevance. This indicates that instruction that creates disbelief or uncertainty that require critical thinking may be a valuable teaching strategy.

When the brain accepts a particular belief, the relationship between beliefs and action is complex (Barcelos, 2006). On a conscious level, as teachers and students 
synthesize new knowledge, beliefs change or are affirmed. Many times, actions reflect new knowledge and current beliefs. Dewey (1933) pointed out that knowing could not be separated from doing. Teacher beliefs are not always consistent with a teacher's pedagogy. Research has not definitively explained how teachers use their belief systems, how beliefs were formed, or how beliefs shape the interaction within classrooms, an exploration of influences on and effects from belief systems will create a more straightforward understanding of human behavior about diversity (Schoenfeld, 1998).

\section{Language Orientations}

The belief systems that teachers embrace determine how they react to linguistic and cultural diversity in the classroom. Heath (1983) asserted that in school, judgments of students are based on the students' ability to respond orally to the teacher in a manner the teacher sees appropriate, typically with mainstream understandings and dominant language usage. Teachers developing professional philosophies about language embedded in linguistic knowledge shape their evaluations of student's language in a positive way (Ball, 2000).

A substantial body of research shows connections between a teacher's negative language beliefs and lower academic achievement and language stigmatizing among students (Ferguson, 1998; Noddings, 2005). Barcelos (2006) explained that teachers often use the "language as a deficit" theory in which "learners are viewed, compared, and judged according to an ideal view" (p. 14). This theory insinuates that there are erroneous ways to use language. If teacher beliefs are oriented in the "deficit" model, they will instruct students as though there is a problem to be fixed. When this happens, teachers tend to perpetuate a subordinate status among nonstandard English speakers 
(Freeman, 1995). However, teachers who believe that language is a resource will build on the linguistic and cultural backgrounds that students already possess and promote further language development (Ruiz, 1984). Students bring funds of knowledge to the classrooms. When teachers believe the resources students bring are valuable, the dominant classroom discourse does not suppress cultural capital (Bourdieu, 1990). Ladson-Billings (1994) stated that teacher beliefs that guide the use of linguistically and culturally responsive pedagogical strategies increase student efficacy, motivation, and academic achievement.

\section{Exploring Beliefs}

All teachers hold beliefs about their work, their students, and their roles and responsibilities (Pajares, 1992). Barcelos (2006) described two concepts of beliefs. One concept characterizes beliefs as ideas that are based on correct or incorrect knowledge, but which produce confidence in actions, representations, or expectations. Another concept describes beliefs as values based on previous experiences and knowledge that hold true in the mind of the believer. Moreover, beliefs cannot be fully explored or changed if the individual and the context in which that belief was established are not considered.

Despite the fact that many scholars have explored teacher/student belief systems in language use and usage and claim it is a very important topic, many areas have not yet been explored (Kono, 2001). Barcelos (2006) describes studies that have explored beliefs as trying to find "truth" from a scientific view point and discover beliefs that are considered to be wrong. Yet, the object of studies exploring teachers' beliefs should not be to find "the" truth with regard to linguistic and educational theories, but to find "their" 
truth because it is the teacher's beliefs that will guide their teaching. It is valuable to explore the beliefs and knowledge that preservice teachers possess in regards to linguistically and culturally diverse students to uncover the teachers' language motivation and language strategies in the classroom (Barcelos, 2006).

\section{Nothing but the Facts Please: Linguistic Knowledge}

As described by Van Driel, Bulte, and Verloop, (2007), Pajares (1992), and others, teachers' knowledge not only includes understandings of cognitive components but also awarenesses and understandings of aspects of culture, child development, educational pedagogy, and human interaction that is acquired through life experiences and formal education. This knowledge involves personal perspectives as well as perspectives held by others.

All teachers should have basic epistemological language understandings including the ability to use and discuss language based on an accurate foundation of linguistic knowledge (ACTFL, 2011; ASHA, 2005; Fillmore \& Snow, 2000; Freeman \& Freeman, 2004; Jensen, 1998; Justice, 2004; Moats, 1994; Ovando, 1997; NAEYC, 2009; NCRCD, 2002; SBEC, 2008; Wilson, 1996; Wood \& Floden, 1990; Wren, 2002). This foundational linguistic knowledge derived through the lenses of articulation, psycholinguistics, neurolinguistics, sociolinguistics, and educational linguistics is described in this chapter (also see Table 2.1).

Types of Knowledge

Language Acquisition Process

Phonology - sounds to form words
Specific Elements of Knowledge

first- and second- language development

auditory and physiological 
phonemes and syllables

pronunciation and prosody

\begin{tabular}{ll} 
Grammar - rules of word/sentence structure & syntax and morphology \\
\hline Semantics - relationship of words/ meanings & prosody and interpretation \\
\hline Pragmatics - language use & communication and context \\
\hline Culture - background, identity & academic and vernacular \\
& registers and dialects \\
\hline Instruction - classroom language & code-switching \\
& developmental process and time \\
Memory - brain function & theory and method \\
\hline
\end{tabular}

Table 2.1 Necessary Linguistic Knowledge for Teachers

\section{LoLiTa - A Trip around the Mouth: An Articulation Perspective}

The articulation of language has a powerful influence on the perceptions people adopt about other people. Judgments and assumptions are made all over the world based on the manner in which people speak. The importance of knowing how sounds are formed and where they are formed in the mouth are essential for many professions. In his book, Lolita, Nabokov used the term symbolically creating a relationship between the girl that he loves and the articulation process. Nabokov (1955) opened his book, "Lolita, light of my life, ... Lo-lee-ta: the tip of the tongue taking a trip of three steps down the palate to tap, at three, on the teeth. Lo. Lee. Ta.” (p. 9). His depiction of the girl, Lolita, is through each of the parts that make up her whole personality. His accounts of the 
pronunciation of her name comprise combining the syllables that form the whole word. Lolita, in the language processing sense, is a word that uses the tip of the tongue to produce sounds in several regions of the mouth from front to back to front again. By using the linguistic understanding of the production of Lolita, Nabakov stresses that as with the human character, language is the whole equaling the sum of the parts.

Teachers often focus on the "correct" pronunciations required in proficient language use but disregard the importance of the how those sounds are produced (Buckingham \& Christman, 2008). The physiology of proficient language production is often ignored in classrooms. For example, many teachers do not enunciate their words to transfer across languages. Students may also be corrected when their pronunciation is not of the standard variety of English. Students coming from a native language that differs from Standard English may not know how to pronounce English sounds. Sound differences in languages affect not only listening, but production as well.

Articulation includes physical production and auditory perception components (Underhill, 1994). Underhill (1994) explained that students move between hearing the language, examining it, and constantly refining their perception of continuous speech. Innis (1986) further described verbal language as an articulated code, which has a 'vocabulary' of basic units together with syntactical rules which can be used to generate larger meaningful combinations. This study attempts to describe the most important components of articulation including the physiology of sound, morphology, phonology, and syntax which influences a student's auditory perception captured by the literature review. Within these categories, this study explores the intentions and perceptions that speakers and listeners have during articulation. 
Though an understanding of articulation, specifically pronunciation, is important for teachers to obtain, accent reduction is not necessarily the goal of this knowledge. Nieto and Bode (2008) used case studies in their work to explain that some students with "thick" accents become frustrated over time when others cannot comprehend them. However, these same students consider their accent a part of their identity and want to retain the ability to access it.

Students have also explained that when first encountering an unfamiliar, nonnative accent, they, as listeners, have problems identifying words and recognizing when mispronunciations have occurred (e.g., Lane, 1963; Schmid \& Yeni-Komshian, 1999). Past research studies suggested that the ability to perceive speech was driven by a process of normalization which removed distortions from the speech signal to allow easy retrieval of linguistic content (Shankweiler, Strange, \& Verbrugge, 1977). This process involved the "stripping away" of the surface characteristics of speech, leaving very basic linguistic units to be considered (e.g., Halle, 1985; Joos, 1948; Neary, 1989). Recent research has uncovered patterns of familiarity-based improvement for accented speech (Sidaras et al., 2009). In other words, a listener should be better able to recognize speech that is accented when they are familiar with this type of accent; and they should improve even more when a familiar speaker produces this accented speech. In sum, compensatory strategies for listeners can help overcome difficulties in perceiving speech (Sidaras et al., 2009).

\section{Physiology of Sound}

From the physiology of speaking to the structure of language, communication is a natural consequence of being human (Hopper \& Naremore, 1973). Sounds are called 
phonemes. Akmajian, Demers, Frmer, and Harnish (2001) found that in normal communication, an average of eight phonemes per second are produced. They also report that during phoneme production, the brain sends signals to the lungs, vocal cords, tongue, and lips to contract or relax. Languages include different collections of sounds and the sounds are arranged in different ways. For example, English has approximately 40 phonemes while Spanish has approximately 22 and Hawaiian 13 (Freeman \& Freeman, 2004).

The study of how these sounds are produced across languages is known as phonetics. Phonetics includes the articulatory features of each phoneme such as the place and manner of articulation (Justice, 2004). The features of phonemes in all languages are explained in a chart known as the International Phonetic Alphabet (IPA) chart. (See Figure 2.) Phonology explores sound differences and the organization of sounds into syllables and words (Ottenheimer, 2009). Phonological development involves learning to organize sound units, syllable structure, rhythm, and phonotactics (i.e., rules of possible phoneme sequences) of a language (Curtin \& Werker, 2009). These sound units must then be used in productive and receptive ways. 


\begin{tabular}{|c|c|c|c|c|c|c|c|c|c|c|c|}
\hline & Bilabial & Labiodental & Dental & Alveolar & Postalveolar & Retroflex & Palatal & Velar & Uvular & \begin{tabular}{|l|} 
Pharyngeal \\
\end{tabular} & Glottal \\
\hline Plosive & $\mathrm{p} b$ & & & $\mathrm{t} d$ & & $t d$ & c 1 & $\mathrm{k} \mathrm{g}$ & $q \mathrm{G}$ & & $?$ \\
\hline Nasal & $\mathrm{m}$ & $\mathrm{m}$ & & $\mathrm{n}$ & & $\eta$ & $\mathrm{n}$ & $\eta$ & $\mathrm{N}$ & & \\
\hline Trill & B & & & $r$ & & & & & $\mathrm{R}$ & & \\
\hline Tap or Flap & & & & f & & r & & & & & \\
\hline Fricative & $\phi \beta$ & f $v$ & $\theta \delta$ & S Z & $\int 3$ & S Z & $c ̧ \mathrm{~d}$ & $x \gamma$ & $\chi \quad \mathrm{b}$ & $\hbar \int$ & $\mathrm{h} h$ \\
\hline \begin{tabular}{|l|} 
Lateral \\
fricative
\end{tabular} & & & & $\$ 3$ & & & & & & & \\
\hline Approximant & & v & & $d$ & & $l$ & $\mathrm{j}$ & $u$ & & & \\
\hline \begin{tabular}{|l} 
Lateral \\
approximant
\end{tabular} & & & & 1 & & l & $\kappa$ & $\mathrm{L}$ & & & \\
\hline
\end{tabular}

Where symbols appear in pairs, the one to the right represents a voiced consonant. shaded areas denote articulations judged impossible.

\section{Figure 2. International Phonetic Alphabet (IPA Chart)}

Note. "The International Phonetic Alphabet," by The International Phonetic Association. Copyright 2005 by International Phonetic Association. Reprinted with permission.

At the beginning of language acquisition children must learn to differentiate sounds - not only speech sounds from non-speech sounds, but also all speech sounds from each other. Many researchers have studied infants to understand how language acquisition develops (Colombo \& Bundy, 1981; Hopper \& Naremore, 1973; Jusczyk, 1997; Karzon, 1985; Lasky, Syrdal-Lasky, \& Klein, 1975; Streeter, 1976). Findings from this research show that before understanding of speech takes place, infants are sensitive to and can discriminate between phonetic speech categories, syllable structure, rhythmicity, and acceptable sound sequences in various languages. These studies illustrate how early our speech parsing capabilities develop and how, even at a young age, language input creates our receptive impressions. It is important to note, if speech 
sounds are differentiated in these manners in infancy, this may have implications for how and when students are taught alphabetic principle, phonics, and so forth.

First and second language acquisition are usually developed in different ways (Krashen, 1982). Native speakers of a language use phonemic distinctions for comprehension while non-speakers focus on phonetic distinctions. According to Pike (1954), the term phonemic represents any unit of sound in a language, at any level, of any kind, which native speakers reacted to as a relevant unit in that context. An example is whether $/ \mathrm{b} /$ and $/ \mathrm{v} /$ make contrast in meaning in a minimal pair in language in which native speakers decipher the accuracy of phoneme identification through shared cultural experiences. He described the term phonetic to represent a study from the outside of a language, by which phoneme analysis relies on externally created concepts and categories usually learned by memorization. In other words, one typically learns a second language using phonetic language development. Some allophonic (i.e., predictable phonetic variant of a phoneme) examples of phonemic and phonetic differences can interfere with comprehension in the classroom. Speakers of some languages create meaningful contrasts out of these differences in articulation. For example, English has $/ r /$ and $/ l /$, which share acoustic properties and are called liquids. Chinese has only /l/. A Chinese speaker may find it difficult to distinguish between the $/ r /$ and $/ l /$ sounds and to produce the sounds distinctly. Another example is the Spanish voiceless /t/. Many Spanish speakers articulate the $/ t /$ instead of $/ \Theta /$ because there is no $/ \Theta /$ (diagraph $<$ th $>$ ) in the Spanish language. Hindi speakers must learn to ignore phonemic contrasts found in Hindi but not in English, while a Hawaiian speaker will have to learn to add phonemes in 
English not present in Hawaiian. A teacher's language behavior can reflect acknowledgment of these differences.

The physiology of speech production involves complex collaboration of the muscle systems that make up the vocal apparatus. Though there are many parts of this system controlling speech, this paper addressed only the vocal tract, larynx configurations, and patterns of airflow briefly. The amount of airflow and movement of the articulatory muscles determines the quality, pitch, loudness, and length of sounds. All markers in speech depend on these variables for phonetic realization and characterize speaker distinctiveness (Laver \& Trudgill, 1979). Additionally, Laver (1968) explained markers such as voice features depend on anatomical differences (i.e., vocal tract length, dimensions of lips, tongue, nasal cavity, pharynx and jaw, dental characteristics, and laryngeal structures, age, gender, and physical size differences) and personal vocal apparatus settings (i.e., vocal folds vibrate to give a nasal, creaky, or whispery sound). These settings also include accent specific language characteristics. Speech production is determined by the language/languages that are spoken by an individual and personal preference early in life, but can also change over time. Awareness of the complexities of language production can minimize verbal communication issues.

\section{Morphology}

Morphology is the study of word formation including word classes (i.e., parts of speech), word function and form (i.e., representation of object or concept), and word meanings (i.e., definitional ambiguities of words). The classification of a word describes the word's "behavior." In addition, a single word can take multiple forms in multiple grammatical uses. Grammatical forms such as affixes can change the meaning. For 
example, a noun, luck with a morpheme "un" and a morpheme "y" changes its property to an adjective, unlucky. Furthermore, pronunciation is affected when the sounds (morphemes and phonemes) are spoken together to make a word. An illustration of this is the pluralization of the word "child." By adding "ren" to the word "child," the pronunciation changes. Systematically, morphemes change the sounds of neighboring morphemes (Justice, 2004). For example, the pronunciation of "jewel" changes when the morpheme "ry" is added creating the word "jewelry." Finally, spoken words without context can create confusion such as homophones. For example, the word, /blu:/ when spoken could be blue or blew.

\section{Phonology}

With regard to the phonology of language, sound production is most commonly influenced by two factors: dialect and prosody (Gumperz \& Hernandez-Chavez, 1972; Freeman \& Freeman, 2004; Snedeker \& Trueswell, 2004). According to Freeman and Freeman (2004), all speakers have a dialect that is either influenced by regional or social features. Additionally, they found that students would acquire the dialect of their teacher. A teacher's belief about language acquisition and the "correct" way to speak will affect their instructional dialect as well as the oral language they accept from their students (Freeman \& Freeman, 2004).

Prosody has a powerful influence on verbal communication. With regard to prosody, pitch (intonation) and stress (accent) are the two most significant influences on cross-cultural communication. Gumperz and Hernandez-Chavez (1972) contended that poor use of these suprasegmental features of language are the most noted cause of misunderstandings. Studies indicate that users of a language share knowledge about the 
relationship between prosody and syntax and are able to use this knowledge to guide their language choices.

Cognitive psychology studies of speakers and listeners in dialogue show that they use local (word level) and global (context level) cues differently. Listeners use prosody during the perception of language to determine word and sentence level meanings. Snedeker and Trueswell (2004) concluded that language learners are able to parse sound, syllable, and word forms out of sentences by using the context. Furthermore, they posit that prosodic organization of an utterance guides interpretation of syntactic structure. The opposite is also true; speakers mark meaning in an utterance through prosody.

Snedeker and Trueswell (2004) also studied major prosodic breaks in various places in sentences. They found that prosodic variation was predominantly influenced by the verb in a sentence. Their study, however, has been controversial because it was almost solely in English. Furthermore, they have found that accessibility to verb usage plays an important role in a listener's ability to establish the intended meaning because the verb reaches the ear very early during the hearing of sentences.

\section{Syntax}

Children must distinguish syntactic arrangements in spoken language. According to Saffran (2003), the distribution of word types in sentences, speech categories, and word boundaries are components of language structures. Many times, despite our desire to speak in complete and well-thought-out sentences, our spoken language is fragmented and incomplete (Wingfield, Peelle, \& Grossman, 2003). Wingfield, Peelle, and Grossman (2003) further explained that though some educators assume that spoken fragments imply incompetence, many times spoken fragments are accepted and 
understood. Spoken fragments, however, may lead to confusion in multilingual students' comprehension. However, even when the surface structure of a sentence changes, some listeners can gain meaning by noting the subject, noun phrase, and verb phrase. Norden (2007) confirms that comprehension is largely determined by grammar and syntax. Bornkessel-Schlesewsky and Schlesewsky (2009) further argue that though most language comprehension relies on surface structure, underlying assumptions about the input have to be made when language is not straightforward. Research has found that the way a person handles syntactic ambiguities offers insight into their language processing (Wingfield, et al, 2003).

\section{Auditory Perception}

The language that teachers use with students must be accessible. From sound discrimination to word recognition to sentence processing development, determining realtime utterances is a rapid computational procedure using syntactic, semantic, and pragmatic features that have an immediate influence on linguistic and non-linguistic comprehension (Trueswell \& Gleitman, 2009). The following section explores language processing from the psycholinguistic perspective.

\section{You Will Know a Word by the Company it Keeps: A Psycholinguistic Perspective}

A teacher's knowledge of how competent language use and language understanding occur is vital to guiding language behavior that diverse student populations can access. Children begin to acquire a foundation in phonology (the sound system), morphology (the rules for word formation), and syntax (the rules for sentence formation) as well as the meaning in language. As it relates to teaching, this study focused on word recognition, semantic representation and conceptual structure, and production of 
language within the field of psycholinguistics. Bornkessel-Schlesewsky and Friederici (2009) have found that the part of the brain responsible for word recognition and language production is the same part of the brain responsible for language comprehension.

\section{Word Recognition}

Semantic knowledge is an important aspect of teachers' language use. Oral language comprehension relies on transparency in speech perception which consists of production and reception components specifically in word recognition. In other words, for a listener to understand a speaker, the speaker must clearly enunciate words providing lexical access to the listener. However, when segmenting sounds in speech to establish word recognition, a mismatch between the input and stored lexical information can prevent comprehension. Marslen-Wilson and Zwitzerlood (1989), for example, studied the mismatch between initial phonemes in words. The conclusions in this study show that initial sounds in an utterance must be distinguished properly for word recognition to occur. In addition, researchers studied the mismatch on final consonants (Frauenfelder, Scholten, \& Content, 2001), polysyllabic word mispronunciation (Gow, 2001), and nonword miscues (Norris, 1994) and found that none of these mismatches constrains lexical access more than initial phoneme and word/non-word mismatch for monolinguals as well as multilingual students.

Furthermore, Lehiste (1972) stated that constraints on lexical access (i.e., word recognition) become more difficult in continuous speech. When speech is produced rapidly, the processing center has less time to interpret. To add to the difficulties of understanding continuous speech, speakers also tend to enunciate each word less and 
make more speech errors that create more non-words than if they were speaking slowly (Dell, 1986). McQueen (2009) contended that in any given utterance, comprehension could occur without explicit and categorical decisions about the identity of every word. When speech is undecipherable, other less reliable cues to word boundaries must be used such as durational evidence, intonation, rhythmic distinctions in syllables, vowel harmony, phonological knowledge, and probabilistic phonotactic knowledge (McQueen, 2009). Furthermore, Dijkstra (2009) found that multilingual speakers also benefit from cues such as cognate and minimal pair features. Additionally, Norris (1994) explained that feedback from the speaker is beneficial to assist the listener in adjusting the interpretation of a speech sound. These cues are helpful to language learners.

\section{Semantic Representation and Conceptual Structure}

After word recognition is achieved, the brain seeks meaning through semantic representation and conceptual structure. There are two opposing theories of semantic representation (Jackendoff, 1983; Miller \& Fellbaum, 1991). The first theory contends that a word's meaning is represented in terms of its relationship with other words by holistic properties. The opposing theory contends that a word's meaning is represented by featural properties such as "definition and characteristics." Vigliocco and Vinson (2009) posited that the combination of these theories allows for predicting comprehension issues to provide equivalent access to concrete and abstract words. According to researchers, concrete words facilitate processing more readily because they typically have more semantic features associated with them and a higher degree of connectivity to other words than do abstract words (de Mornay Davies \& Funnell, 2000; Gentner, 1981). Teachers can use more paralinguistic cues to define words. These researchers further 
explained that a higher degree of connectivity to the context allows for a greater development of language.

Most commonly, the semantics of language is studied at the single word level, as larger linguistic units create concern for accuracy (Vigliocco \& Vinson, 2009). Researchers have explained the relationship between words explicitly while leaving the relationship between semantic and conceptual representation implicit. According to Lindfors (1991), children know that language expresses meaning, but sometimes do not derive the same meaning the speaker intended. For example, teachers often ask children to "repeat after me." It is not uncommon for children to make the appropriate noises with little or no concept relating to those noises. It can also be confusing when spoken words that sound the same have different meanings. Cruse (1986) explained that not only does polysemy affect the understanding of words, but also multiple concepts for each word complicate issues cross-linguistically.

The semantics of language can also be convoluted by the choice of language. Pinker (2007) argued that using the art of noise, people could shape ideas and thoughts in each other's minds with exquisite precision. He explains that by maneuvering sounds, people can change the meaning of the images they create with language. In contrast, examples published in the Quarterly Review of Doublespeak document how vague or misleading spoken language can also obscure meaning (National Council of Teachers of English, 1993). Provided in the review are quotes from educated speakers who speak loudly, but say nothing meaningful or relevant. The use of academic language in instruction provides another example of possible influences on the ambiguity of meaning. For instance, students often cannot understand instruction because they lack access to 
academic English. In sum, if language is not used with precision, the lack of understanding on the listener's part negates the intent of the speaker.

Further compounding the understanding of conceptual structure is the issue of code-switching. The ability to code -switch is thought, by some, to be one way multilingual students can transfer their concepts of the world using the knowledge of their many languages (Gumperz \& Hernandez-Chavez, 1972). Multilinguals often tend to code-switch between the languages they speak. Slobin (1973) found that children use their multiple languages differently in different contexts. Gumperz and HernandezChavez (1972) argued that people choose a specific language that they associate with that language's experiences. They also found that people choose the specific language that can best describe the concepts they want to convey. Additionally, Lindfors (1991) found that people use a certain language because the syntactic devices for expressing things are simpler in certain languages and students tend to master the simpler language first. Changing between languages can alter the semantic representation and conceptual structure of spoken language.

\section{Production of Language}

Finally, considerations for the production of language include word production and speech production at the sentence level. According to Costa and Caramazza (1999), at the word level, production includes transferring conceptual representation to the phonological representation to articulatory representation during the speaking process. The opposite process is reception. During language production, researchers found that listening as well as speaking in multiple languages at once took longer than when done in one language at a time (MacNamara, Krauthammer, \& Bolgar, 1968). If a teacher or 
educational institution chooses to implement bilingual instruction, time and proper organization are important influential factors to students' gaining of cognitive control (Dijkstra, 2009).

Fowler (2009) suggested that speech production requires composing a plan for an utterance and articulating what the talker intends to publicly display to convey an acoustic signal. Linguists stress that prelexical processing is required to solve any invariance processing (Scharenborg, Norris, den Bosch, \& McQueen, 2005). When a speaker uses another language, a language cue is contained in the preverbal message as well.

Following the planning phase of word production, prosody, at the word level and at the sentence level, is a factor that influences the acoustic signal as much as word choice and grammar. Intonation, contour (i.e., frequency), and articulatory strength (i.e., lengthening and pausing) causes cross-linguistic confusion (McQueen, 2009). Cutler and Otake (1999) compared prosody at the word level in Japanese speakers and English speakers for lexical access. Additionally, Fox and Unkefer (1985) compared prosody in Mandarin and English speakers. These studies found that speakers who spoke both languages could not use stress patterns to gain comprehension in either language. For example, Mandarin speakers were not familiar with the sentence level stress pattern (i.e., rise in pitch) in English that changes a statement to a question. Moreover, the distinctions in stress were not transferable. Conversely, English speakers did not exhibit brain activity when hearing tones in tonal languages (Buckingham \& Christman, 2008).

Many times difference in prosody at the sentence level changes the perception of syntax. For example, variation in prosody can distinguish a question from a statement. 
Richards (2010) believed in a universal principle of prosody meaning that prosody is integral to the syntax of a sentence whether it is overt or covert in its forming of questions. He also believed that the pitch of a question peaks wherever the $w h$-word (what, where, when, why) occurs. Bornkessel- Schlesewsky and Schlesewsky (2009) also found that according to the perception of the prosody that the $w h$-word carries, different parts of the brain are activated to comprehend the language. For example, if an English speaker asks a question, the $w h$-word pitch change will signal a question. However, if the wh-word is spoken in a statement such as, "What the data tell us is...," the listener will expect a statement according to the prosody used in the word and sentence.

To understand language reception and production, uncovering universal characteristics of the language system as well as language-specific properties is important (Costa et al, 2009). All languages have structured systems and few have truly unique features that no other language shares (Richards, 2010). Researchers have established that the use of determiners in the selection of closed-class words (i.e., words that serve a grammatical function) is different across languages (Costa, Alario, \& Sebstian-Galles, 2009). An example in English is how the retrieval system of the definite article the depends entirely on semantic information. However, in Germanic and Slavic languages, for example, the retrieval of determiners also depends on grammatical gender. The use of cross-linguistic research can explain the relationship between language universals and specific properties and how they affect speech reception and production. 


\section{Language behind the Brow Ridge: A Neurolinguistic Perspective}

The field of neurolinguistics has produced information that provides teachers with a better understanding of how the brain processes language. Norden (2007) states that language use is a higher-order cognitive function. A long history of research in medicine and philosophy indicated language processing involved only specified areas of the brain (Bornkessel-Schlesewsky and Schlesewsky, 2009). Contrary to past beliefs, certain areas of the brain can no longer be associated with specific language functions (BornkesselSchlesewsky \& Friederici, 2009; Jensen, 1998; Sousa, 2010). Years ago, it was believed that Broca's area, responsible for production of language, and Wernicke's area, responsible for understanding spoken language, were the primary areas of the brain used in language use. New research suggests that the sensory and motor areas of the cerebral cortex are also involved in language use (Norden, 2007). Norden explains that the auditory areas are involved in the ability to interpret spoken language as meaningful while the motor areas are involved in the ability to produce combinations of sounds of language that are meaningful to listeners. Stemmer (2008) revealed one possible reason for this. Her studies of joke comprehension found that several areas of the brain used for processing several elements of language are involved in processing each utterance. In addition to cognitive elements of language such as the generation of inferences based on contextual, experiential, or knowledge factors, there are other elements such as surprise, apprehension, or appreciation involved. The link between words and the associations individuals make with those words may explain neural activity throughout the brain when processing language. 
The primary language processing areas of the brain are the left perisylvian cortex, especially Broca's and Wernicke's areas near the primary articulatory motor and auditory cortex. Perfetti and Frishkoff (2008) found, however, that some linguistic processing such as non-literal inference comprehension occurs in the right-brain hemisphere as well. Word recognition is an example of left-brain processing while prosody is associated with the right brain circuits (Norden, 2007).

According to Bornkessel-Schlesewsky and Friederici (2009), language is processed in many areas of the brain, sometimes independently and sometimes simultaneously. For example, word recognition and syntactic arrangement maybe processed independently, but word recognition and semantic representation maybe be processed simultaneously. In addition, phonetics and phonology errors have been found to exist in separate parts of the brain, but patterns of brain activation support the idea that integration of phonetics and phonological processing occurs across the language cortex. Commonly, phonetic errors are misinterpreted as phonological errors even though "errors arise from the intermediate stage of production, subsequent to the phonological selection and sequencing of phonemes, but prior to actual articulatory execution" (Buckingham \& Christman, 2008, p. 129). Many educators do not have the knowledge to analyze language errors accurately. As a result, many students are placed in special education services or intense phonics programs (Allington, 1984).

In addition, Pulvermuller, (2009) found that recent studies have even been able to account for processing times in various areas of the brain. For example, phonological information like stress, duration, and vowel quality is almost immediately accessed. Lexico-syntactic properties like word class are accessed in 150 milliseconds (ms). 
Semantic information can be accessed as quickly as $250 \mathrm{~ms}$. Syntactic reanalysis happens in approximately $450 \mathrm{~ms}$. Neurolinguists have determined that the brain develops near-simultaneous access to language information over time (Pulvermuller, 2009). Whether the brain is processing multiple languages or specific lexical information in one language, the human brain has the capacity for automaticity according to these studies (Bornkessel-Schlesewsky \& Friederici, 2009; Pulvermuller, 2009).

Nickels' (2008) model of language processing is based on the premise that speech input and output occur in different parts of the brain. The stages of language processing included in Nickels' model are hearing a sound (i.e., auditory component), parsing the sounds (i.e., acoustic input), creating meaning from the sounds (i.e., semantics embedded in memory), and articulating the sound (i.e., language production). (See Figure 3.) 


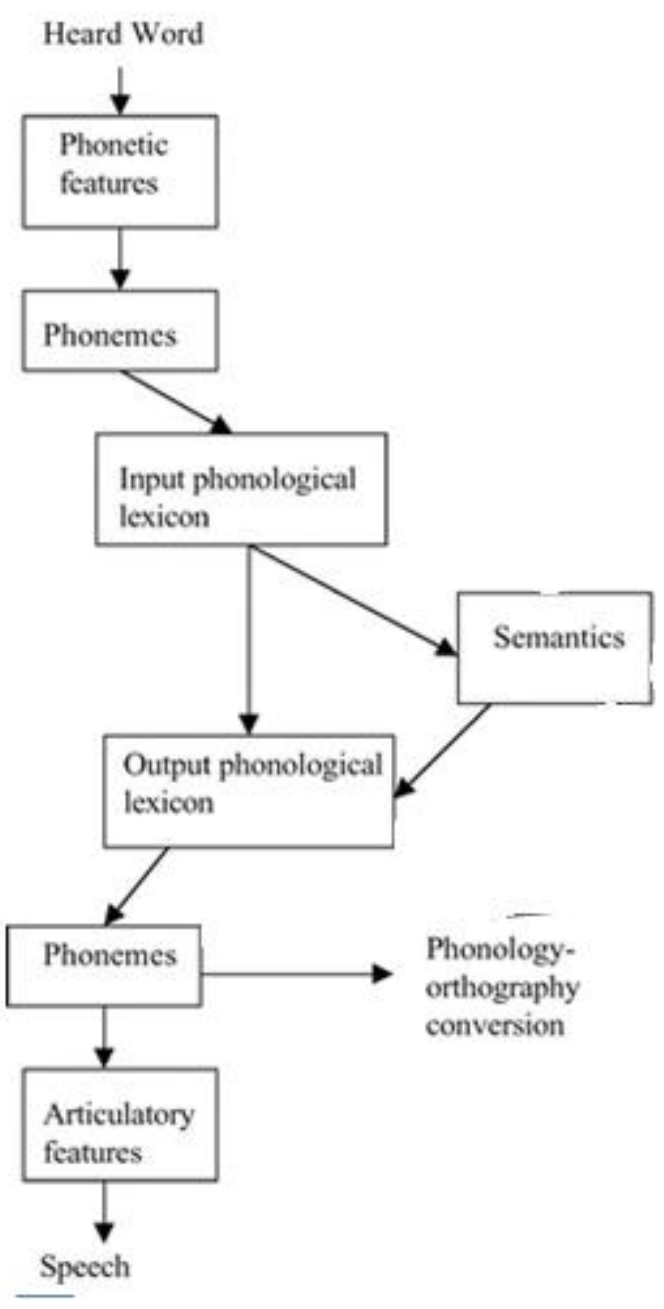

Figure 3. Model of Language Processing in the Brain

Note. "Language Processing Model," by L. Nickels, 2008, Handbook of the Neuroscience of Language, p. 15. Copyright 2008 by L. Nickels. Reprinted with permission.

\section{Auditory Component}

Humans routinely encounter complex sounds of spoken language (Horwitz \& Wise, 2008). Generally speaking, there are two dominant auditory fields of the brain used for speech: spectral and temporal. These areas allow the detection of phonemes, syllables, stress, and variations in amplitude and pitch. This verbal information leads to linguistic and non-linguistic comprehension by allowing the listener to deduce the 
meaning of spoken language as well as sex, age, intent, and individual identity of the speaker. Even with considerable distortions to speech or the blending of phonemes in continuous speech, some of the discourse heard is comprehensible to the native speaker (Horwitz \& Wise, 2008; Lindfors, 1991). However, to the non-native speaker, any distortion in speech including pronunciation, stress, or vocabulary differences can lead to completely incomprehensible data.

Jensen (1998) explained that without distortions, the auditory cortex allows distinctive sounds to become part of a set of recognizable sounds and accents that will reside in the brain. He further stated that the perception and production of language sounds, individual identities, and environmental influences provide the brain's wiring that influences the malleable brain's language perception. Over time, the flexibility in the brain allows the auditory cortex to negotiate the distortions in language to decipher them.

\section{Analyzing Input}

Exposure to speech in any language at a very early age, possibly prenatal, activates specialized areas of the brain for perception (Pena et al, 2003). "There is similarity in the way we produce and perceive sounds because the human neurological and vocal apparatus used in speech is architecturally and structurally universal" (LippiGreen, 1997, p. 13). Additionally, left-hemispheric dominance plays a key role in specializing human language abilities (Pulvermuller, 2008). However, individual genes and environment can create some variation in the structure of the brain (Jensen, 1998). Additionally, heredity provides 30 to 60 percent of the brain's wiring while the environmental impact is 40 to 70 percent (Healy, 1990). 


\section{Meaning through Memory}

Regardless of the language(s) spoken, the brain attempts to create meaning through memory systems (Jenson, 1998). When encountering spoken language, the brain takes what is heard and compares it with what is stored in memory. Chomsky (1968) argued that humans are born with basic structures of all human languages already present in the brain. "The way language works, then, is that each person's brain contains a lexicon of words and the concepts that they stand for (a mental dictionary) and a set of rules that combine the words to convey relationships among concepts (a mental grammar)" (Pinker, 2007, p. 76). Many parts of biology are considered important like the brain structure, neurons, molecules, and genes when examining language processing. To learn a language, a child must learn to put words together coherently, not by recoding which word follows which word, but by recording which word category follows which word category through the complex relationship between biological structures.

According to Dijkstra (2008), when speaking a first or second language, the mental lexicon stores all available knowledge of the words - the orthography, phonology, concepts/semantics, pragmatics, morphology, and the language to which the word belongs. Gonzales (1984) further explained that a student's knowledge of the vocabulary, syntax, and semantics develops gradually; though early in life humans possess some type of language competence. Current research on the biocognition of language involves mapping the lexicon to the brain (Ullman, 2008). Our lexicon is stored in different parts of our brain and language processing is complex. Therefore, language cannot be taught to a specific part of the brain or in a specific way for everyone as previously believed. 
As language becomes learned, the brain uses two types of memory systems for storage, declarative or procedural memory (Ullman, 2008). Declarative memory usually contains semantic and episodic knowledge. Typically, knowledge in this memory system is explicitly and rapidly learned including simple words, irregular morphology, and syntax. Procedural memory contains learned rules and sequences. Knowledge in this system requires repeated exposure to stimuli and is sometimes referred to as "implicit memory." Mental grammar such as syntax, regular inflected morphology that can be generalizable, and phonology such as novel words is stored in procedural memory. Structural characteristics of each language determine what aspect of language is stored in each memory system and, therefore, how each language is processed. For example, Paradis (2008) studied bilingual speakers' language processing in relation to memory systems. He found that Greek is a morphologically rich language and typically requires the use of different memory storage than for English, which is morphologically poor in comparison. Additionally, irregular verb morphology is declarative and can be recalled from memory as whole, unanalyzed items in English. The opposite is true in SpanishCatalan speakers because irregular verb forms are used with regular forms and are stored in procedural memory. The recall for each language is different and the acquisition in the brain is also different.

Ullman (2008) also observed gender differences in memory. He found that females appear to have superior declarative memory abilities showing a tendency to memorize complex forms of language (e.g., walked), whereas, males tend to compose language with mental grammar using procedural memory (e.g., walk + ed). Given the two memory systems at work in human brains, teachers should be aware that what is 
generally true in their own language might not apply in the same way in the native language of the student.

In recent years, research has focused on memory deficits by exploring linguistic aphasiology. Caramazza, Berndt, Basili, and Koller, (1981) found that aphasics had a strong relationship between short-term memory deficits and syntactic comprehension difficulties. They proposed that simply narrowing the number of words spoken was not enough to eliminate comprehension difficulties due to limited phonological capacity. Further, Saffran (2003) determined that semantic difficulties are associated with longterm memory. During sentence processing, syntactical and semantic features appear to be processed independently (Breedin \& Saffran, 1999). Researchers have used knowledge of aphasiatic language errors to provide training in language processing to nonnative speakers of English in an attempt to increase short-term and long-term memory capacities for language learning.

At one time, scholars posited that for memory storage, specific parts of the brain were responsible for specific aspects of language with each language spoken. More recently, Nilipourn and Ashayeri (1989) suggested that nonoverlapping cortical representations exist for multiple languages. Moreover, research provides evidence that mapping in the brain between the first and second language is created through association patterns. Albert and Obler (1978) suggested overlapping language representation in the brain. Additionally, Cummins (1981), concludes that prior acquired knowledge and skills in the native language(s) transfer to the L2. In other words, a nonnative English speaker learning new English vocabulary will associate the L2 with the L1 creating the ability to remember and manipulate new language systems. This association suggests one reason 
that it is important for teachers to understand language acquisition because many teachers tend to either ignore a student's L2 or choose to speak only English in the classroom.

Bilingual language behavior in the brain during language processing is still controversial. Gonzales (1984) believed there is a functional separation of neurological systems between the two languages of bilinguals. Redlinger and Park (1980) believe that one system guides all language learning in the brain, and that there is an interdependence of language use in the brain. Linguistic interdependence seems to be supported by neurolinguistics studies of multilingual aphasia. Not only has research discovered that in early language development mixing and switching of languages occurs frequently (Gekoski, 1980; Gonzalez, 1984; Lindholm \& Padilla, 1978), but after brain trauma and other neurological disorders, multilingual speakers often switch languages in a single conversation even when speaking to monolingual speakers (Abutalebi, Miozzo, \& Cappa, 2000; Riccardi, Fabbro, \& Obler, 2004; Rossi, Denes, \& Bastiaanse, 2003). Research also indicates that the structure of the languages spoken do not determine the type of switching that would occur (Paradis, 2008). The switching and mixing of language could be due to declarative and procedural memory or short term and long term memory, but is as of yet the research is still not definitive.

Many of our deep meanings in life are built through experiences and culture (Jensen, 1998). Our experiences and the culture(s) we identify with influence our use of emotions, associations, and patterns that transfer information to our long term memory. The release of emotion-based chemicals can actually change the physical structure of the brain. Given the diversity of the brain areas that carry meaning, the concept of meaning must also be diverse. Through relevance, meaning is simply "connected" with nearby 
neurons (Jensen, 1998). Every thought you think increases the chance of connections. The greater the relevant associations, the more neural territories involved, the more firmly embedded in the mind the information becomes. Coward (1990) contended that relevance is created through the brain's ability to find patterns. Further, human brains are constantly searching for patterns to make sense of the world's information. Pattern making is thought to be innate. Meaning is thought to come from our brain's capacity to make familiar, relevant connections and form neural networks, which organize patterns (Freeman, 1995).

\section{Articulating Sound (Language Production)}

Just as the human brain looks for patterns in spoken language to decipher meaning, it also creates patterns for language production from language systems. Sousa (2010) asserted that as early as two-months old, language production areas in an infant's brain become active. He estimates that around 18 to 20 months, an infant's language production areas drastically increase activity to learn and produce about 10 new words a day. Although language production areas of the brain are active throughout life, this intense increase in activity lasts up through the age of five-years old.

Current research finds that mirror neurons help to produce rhythmic air patterns via mouth and tongue movements. These sound patterns activate listener's auditory and brain areas that are interpreted verbally. Though listeners cannot see what is occurring inside the speaker's mouth, Sousa (2010) explained that mirror neurons help children learn to speak. These neurons in the brain observe the language process and help produce overt or covert imitations of language. He further explains that speech is a complex motor activity. Over time, the child who imitates through "babble" learns to utter 
phonemes correctly and eventually practices smooth speech. In other words, as language is learned, familiarity with language allows anticipation of future language use. For example, when a speaker stops midsentence, a listener familiar with the language can often complete the sentence.

It is important for educators to learn how to use neurolinguistic discoveries about language processing, and, more specifically memory, processing pathways, and pattern relationships in language.

Therefore, growing a new breed of specialists, who have mastered the art of teaching grounded in the mind and brain research-based science of learning, appears to be one of the roadblocks for a meaningful connection between mind, brain, and educational practice. Newly emerging hybrid field of neuroeducators capable of addressing the issues from a variety of perspectives and in a way accessible to conventional teaching staff and students is important (Summak, Summak, \& Summak, 2010, p. 1646).

\section{Language Roots: A Sociolinguistics Perspective}

From a sociolinguistic perspective, beliefs and knowledge about language, society, and language variation influence a teacher's discourse. Within each of these categories are specific issues dealing with local environment, culture, and identity noted by social markers, accents and dialects, individual speech characteristics, and situational variables. It is also held that the complexities of language, social aspects of language, and influences on languages shape language use in society (Fill, 2007).

The Complexity of the Language Acquisition Process. Language influences and is influenced by many aspects of life; similarly the ability to use and understand one 
language - much less multiple languages - is a complex process. Regardless of whether one believes that language acquisition is predominantly innate; that the genetic structure determines speaking ability (Chomsky, 1968) or in the theory that environmental stimuli trigger the blank slate that humans are born with, causing speaking ability (Skinner, 1957) or in a variation of these theories, it is clear that the system of human language is limitless.

Imagine that you are faced with the following challenge: You must discover the underlying structure of an immense system that contains tens of thousands of pieces, all generated by combining a small set of elements in various ways. These pieces, in turn, can be combined in an infinite number of ways, although only a subset of those combinations is actually correct. However, the subset that is correct is itself infinite (Saffran, 2003, p. 110).

The process of acquiring the system is as complex as the system itself. Linguistic systems around the world share deep similarities and vary in non-arbitrary ways. All people without disabilities learn to use these systems and convey meaning across communities (Pinker, 2007). It is important to understand the intricacies of one language as well as the benefits associated with multiple languages. Words not only name things, they carry layered, dynamic, culturally specific associations, beliefs, and values usually derived from the context in which they are used or the activities with which they are used (Gumperz \& Hernandez-Chavez, 1972). When the individual or context requires switching between languages, the complexity of language use is compounded with what is generally called code-switching. 
Code-switching is a complex phenomenon that occurs when humans transition from one language to another or from formal to informal registers. On one hand, the linguistic signs of codes differ while the social implications are similar. On the other hand, bilingualism as a phenomenon indicates certain linguistic features are similar, but there is a difference in social significance (Gumperz \& Hernandez-Chavez, 1972). Understanding those changes in social significance are a part of moving from one language to another or code switching. This social significance is directly connected to the community that speaks the language.

Each language is tailored to its community of speakers and each language changes in pace with the demands of the speakers (Lippi-Green, 1997). Language varies for every speaker in terms of speech sounds, sound patterns, word and sentence structure, intonation, and meaning from utterance to utterance. "Heterogeneity is an integral part of the linguistic economy of the community, necessary to satisfy the linguistic demands of everyday life" (Labov, 1982, p. 17) However, "the variety of language spoken cannot predict the effectiveness of the message due to the social evaluation and willingness to hear of the listener" (Lippi-Green, 1997, p. 17). The underlying conceptions of a language community and the methods for approaching and quantifying the community's language show need for teachers to understand language variation and further the dynamic between identity and variation.

The relationship between cultural identity and linguistic variation encompasses space, time, gender, age, social status, tenor (interpersonal dynamics of discourse), mode of discourse, and geographic loyalties. According to Labov (1994), variation in language occurs due to three factors: first, language-internal pressures, which include personal 
production and perception; second, language-external influences that come from social behavior norms and other social pressures; and third, variation that arises from language as a creative vehicle of expression. Grammatical, lexical, and phonological variation are available to speakers in infinite variety. Speakers tend to select variation based on social identity more often than on the communicative functions the language happens to serve (Chambers, 1995; Lippi-Green, 1997). Prosodic patterning is a significant way in which listeners determine a speaker's identity (Scollon \& Scollon, 2001). Lexical and grammatical properties must be mutually intelligible for social practices to be carried out. Language variation can sometimes inhibit the possibilities of mutually intelligent speech.

Though linguists typically study language in a broad sense such as "English" or "Russian," today's classrooms tend to have issues relating to specific aspects of language variation like particular registers and dialects (Gee, 2001). Language standardization was devised in an attempt to alleviate these specific language issues created by language variation (Lippi, 1997; Romaine, 2001). Though most of today's approximately 7000 world languages have no standard form, English in the United States became highly codified and prescribed (Romaine, 2007). Positive and negative consequences of language standardization have been scrutinized for years. Milroy and Milroy (1999) indicated that standardization creates shared practices, beliefs, attitudes, and discourses that promote linguistic unity and access to all. Alternatively, Smith (2002) argued that standardization created the ideology that language use is correct or corrupt and produces structures of domination and hierarchy. Appropriacy arguments rationalize the process by which languages of peripheralized or stigmatized groups are simultaneously acknowledged and rejected (Lippi-Green, 1997). The inability to use or recognize the 
social markings of linguistic variants is one of the most significant problems of second language learners, and one that is rarely dealt with in the classroom, where the myth of standard language has a stronghold. The adherence to the use of a standardized language in schools neglects to recognize the intricacies of language use such as accent variation and even correct English spelling. Attempts at creating a standard spelling, stem from attempts at standardizing pronunciation (Ottenheimer, 2009). As Heath (1986) argued, each cultural group has distinctive genres in speech that occur. The genres are patterned so the whole group can interpret speech based on prosody or opening formulae creating shared meanings. This often means that students of the new language are left to adapt to a range of registers and there is a lack of explicit instruction for negotiating these registers (Baker, 2002). By working with the ethnolinguistic communities involved, an educator can learn to be aware of the different language patterns as well as consciously give students opportunities to acquire various genres and registers of language.

Language as a Social Construct. Language is a product of the social process (Levi-Strauss, 1968; Piaget, 1959; Saussure, 1966; Vygotsky, 1978). Saussure's (1966) statement, "Language is a social fact" described how society and language work together (p. 6). He said that language defines society's limits and boundaries and lets individuals say what they want within those confines. By learning the rules of language and then individualizing the language, society plays a role in personal language use and eventually influences the society's language use. Despite this cyclical creation of societal understandings, Saussure's work expands the notion of how even within a community, a speaker's intention may not be a hearer's perception. He explained that although the sign 
(i.e., thought) may be heard, the signifier (i.e., actual utterance said) might not have been signified (i.e., with actual intended meaning).

Along the same lines, the way language is used relates to a person's social status and cultural identity. Because linguists believe that studying language or studying through language requires context, an examination of what people do with language and how what they do is shaped by culture is key in teaching students to use their linguistic resources (Halliday, 1975; Moll, Saez, \& Dworin, 2001; Scribner \& Cole, 1981). Malinowski (1944) believed all aspects of culture are intertwined and called the relationship, "the context of situation." According to Malinowski, language served two purposes within the context of situation. First, language is used for communication of thought, and second, language is used for "communion" which leads to personal and collective action. Communion creates ties between the people of a culture including the reciprocating relationships people call "social capital” (Coleman \& Hoffer, 2011).

Influences on Language Use. Despite the increasing diversity of the student population, few preservice teachers are able to understand students' use of diverse forms of language or conceptualize an active role in developing students' use of language (Lindfors, 1991). Teachers are also often unable to see the bias behind their decisions to accept or deny these diverse language forms (Delpit, 1995; Heath, 1983). Consequently, these diverse uses of language are not aligned with the teachers' academic language and this either lowers expectations or creates negative evaluations from teachers and other students. Major influences on language use and its impact on learning include attitudes about language, the teacher's role, the classroom environment, and individual preferences. 
Traditionally, sociolinguistics considered communicative behavior to be a function of attitudes toward language (Gallois, Watson, \& Brabant, 2007). Researchers found that the impression the speaker creates is more powerful than the speaker's actual behavior. Furthermore, researchers found that when listening to speakers in monolingual contexts, beliefs emphasized favor in "like" languages and social class indicated by “educated" accents. In multilingual contexts, attitudes were positive toward male speakers and speakers of similar language to the listener (Giles \& Robinson, 1990). These findings confirmed that language bias and gender are powerful, even if unconscious, determiners of acceptance and comprehension.

Attitudes about language are also thought to be formulated when interacting through talk with others (Gallois et al, 2007). Scholars studying language issues believe that from the first utterance of discourse, beliefs and attitudes are formed about the speaker. Along with personal interaction across social groups, researchers study contextual and intergroup history and relationships to determine language beliefs.

Wynne (2002) argued that past attitude norms promote language supremacy and oppression. She added that language bias allows some speakers to internalize linguistic inadequacy while depriving some people of cognitive development that allows us to hear people of all cultures. Assumptions based on accents and dialects can be misleading and have resulted in the belief that speakers with certain accents or dialects have cognitive deficiencies (Ravitch, 2000). However, people can say things using a variety of syntax structure and vocabulary in many different accents while the meaning of the utterance remains the same. 
A commonly held practice for many teachers is to ignore the experiential backgrounds of the diverse populations present in classrooms; this practice reflects beliefs that the home language or culture is irrelevant to learning in school (Nocon \& Cole, 2009). According to Ottenheimer (2009), there are political, social, and personal reasons for the lack of attention to diversity in the classroom. First, for some teachers, administrators, and community members, classroom communities are perceived to be effective only when standard language is used. Besides, instructing students from many different language backgrounds in more than one language may create a situation where things might get lost in translation. Second, Ottenheimer (2009) believed the fear of losing one's own frame of reference and sense of culture when attempting to facilitate learning and hesitancy and misinformation about the use of multiple languages drives teachers to use only one standard language - or what they perceive to be one standard language, namely their own. Third, there are many benefits to being multilingual, however; teachers find it difficult to instruct students having a variety of language background (Lippi-Green, 1997; Ruiz, 1984). The lack of attention to linguistic diversity promotes a "language-as-a-problem" orientation. However, linguistic diversity is a resource that can enhance social understandings in classrooms (Nocon \& Cole, 2009). According to Ruiz (1984), "language is a resource to be managed, developed, and conserved" (p. 28).

With the teacher functioning as the predominant speaker in a classroom, it is important that the teacher's intentions are clear in each of what Grant described as the teacher's "moves, structuring, soliciting, responding, and reacting" (Grant \& Grant, 1976). It has long been believed that the teacher exerts the most influence on the social 
environment of the classroom (Halliday, 1975; Smith, 1988). The teacher does so by playing a major role in the communication processes of the human being becoming a social creature. Though there are several factors, language provides the dominant framework for this process. It is not solely through explicit instruction that a child learns his or her culture or values or social organization; it is through accumulated experience with patterns of language. This is true in the classroom as well as the outside world. Teachers facilitate classroom communities in which individuals establish ways of speaking together and at least for a time understand language use within this community as well as within a larger speech community. "In many instances of SLA [second language acquisition] with the aid of formal instruction, including both foreign and second language learning, it is reasonable to assume that what goes on in the classrooms, including the teacher's role in this, is the single most crucial element in determining how students perform" (Long, 1985, p. 4). Teachers must have awareness of the how their language choices affect language use in the learning environment.

The environment that the teacher sets up in the classroom is important. The environment includes the teachers' communication structure as well as the students. Fillmore (1985) explained the use of peer interaction. Teachers must be aware of the benefits or detriments in communication differences in monolingual and multilingual students. She argued that in many classrooms, the teacher is the only one who is proficient enough in English to be helpful to English language learners. She said that students who speak a language other than English might converse primarily in their first language rather than English meaning that English language learners do not get enough practice time with native speakers or that students who interact with other students who 
do not speak English proficiently might learn incorrect or inappropriate English. In many classrooms, communication between multilingual students is significant because these other students explain, translate, amplify, and modify what the teacher says (Henry, 1978). Miller (2009) added that students imitate what they hear and should be exposed to good modeling; however, correction and training do not necessarily improve proficiency. The amount and quality of learning varies greatly depending on the development levels of student and teacher interactions.

Beliefs, culture, environment, personal preference, and language variation affect language use in every person. "The way individuals situate themselves in relationship to others, the way they group themselves, the powers they claim for themselves and the powers they stipulate to others are all embedded in language" (Lippi-Green, 1997, p. 31). Linguistically, teachers play an important role in teaching students the power of language.

\section{In Celebration of Language: An Educational Linguistic Perspective}

According to Spolsky (1978), educational linguistics draws equally on the fields of applied linguistics, psychology, neurobiology, sociology, and education. Theory must originate from issues that directly arise in classrooms. Teachers need to have an understanding - one founded on reliable linguistic theory - of explicitly how language works to resolve linguistic issues that may occur in practice (Hudson, 2010). Through these frames, a holistic picture of teacher language use can yield a better understanding of inclusionary techniques to create effective educational environments.

To understand how language works in the classroom, it is beneficial to establish what populations exist in classrooms first, and then explore prevalent influences on 
language use. Language used in the classroom must keep the learner's needs and limitations in mind to be comprehensible to the learner. Teacher and student populations come to classrooms with different understandings of language systems and different forms of language use. Fillmore (1985) suggested that the language of a teacher conveys subject matter as well as characteristics of the source of input that students need to learn language and learn from language. She pointed to the importance of viewing this diversity as a resource. She explained that teachers who do have knowledge of language systems, subscribe to diversity as a resource theory and do use this language knowledge in the classroom have increased mutual comprehension with students and fewer student referrals to special education. Language in today's classrooms is influenced by differences in teacher and student backgrounds, language structures, and instructional approaches.

Teacher and Student Background Discontinuity. Artiles, Barreto, and Pena (1998) explained that there is an increasing discontinuity between the sociocultural backgrounds of teachers and students. They believed this is one important factor in low academic performance of culturally diverse students. Rao (2005) added that another important factor is the discontinuity between the linguistic backgrounds of teachers and students. For example, many teachers are monolingual, but they are teaching in multilingual classrooms. Moreover, Safford and Kelly (2010) indicated that when multilingual teachers are represented in the classroom, these teachers are prevented from activating their expertise in language and culture due to institutional policy and lack of recognition. Safford and Kelly (2010) interviewed prospective multilingual teachers about their language knowledge and use in the classroom. These teachers revealed 
significant knowledge of language, but felt they seldom used their knowledge in the classroom. Many of these teachers stated that they simply did not know how to apply their linguistic knowledge in the classroom with curricula constraints.

Similarly, another form of discontinuity in the classroom is between the students. "While obvious, it is critical to recognize that students classified as ELL [English Language Learners] are hardly a homogenous group. They come to school with different family backgrounds and varying proficiency in their first language" as well as many other differing characteristics (Gitomer, Andal, \& Davison, 2005, p. 4). Despite variation among students and programs, most state and district reporting related to student outcomes simply examines the performance of all ELLs as a single group resulting in possible misinterpretations of the data. It should be stressed that there is no one right way to speak, only appropriate ways in certain situations to allow for understanding to occur. A discussion of different talk types can be instrumental in helping teachers and students gain linguistic knowledge and eliminate miscommunication issues.

The discontinuity between teachers and students can lead to miscommunication for several reasons. For example, pronunciation differences influence the dialogue in classrooms as stated previously in this chapter. However, a teacher's speech can provide a model for pronunciation. Miller (2009) asserted that when the teacher has had pronunciation training, students benefit from indirect and direct pronunciation training. Miller suggested that to alleviate misunderstandings, situational cues and patterns, and repetition with grammatically appropriate language that is tailored to a student's proficiency level aid in more rapid acquisition and comprehension. Kohl (2002) gave an example of miscommunication that could have to do with cultural disagreement. He 
cited a girl who fully understood what was required of her, but simply did not agree with the instructions. Her answers to questions were "incorrect" according to the curriculum based on the premise of the questions. When she verbally articulated her disagreement; however, the teacher found that the six-year-old knew the "correct" answer that was expected from her. Yet, the student did not philosophically agree with the answer. At that point, the teacher had the information necessary to proceed with proper instruction. "Students are very sensitive to the language of their teachers - the words, the tone, its trustworthiness - while teachers are insufficiently aware of how they are being heard and understood" (Kohl, 2002, p. 146). Through analysis and reciprocal speech, teachers develop an awareness of how they are presenting themselves. Similarly, teachers can construct other means of communicative understanding in the classroom. If students cannot understand a teacher's language, students can benefit by receiving nonverbal communication or cooperative group work.

Along the same lines, Kadeghe (2000) found that concepts and content were articulated more effectively for some by using code switching as a pedagogical tool to remediate miscommunication. Studies in other countries such as Africa found that lecture in one language and discussion in another has even led to further exploration of educational policies. Conversely, in the field of education in countries such as the United States, monolingual normativity may be a goal, but not an empirical reality (Blommaert, 2007). As teachers and students explore language use in the classroom together, best practice can meet individual need as well as the needs of the class. 
Past analysis of language in a classroom has included not only teacher's attention to children's language use, but also teacher's attention to their own usage and grammar, though there are limited studies. Although teachers are often aware of implicit and explicit strategies of using language in general; they are also many times unaware of their own use of language in a diverse classroom. Language is influenced by personal style, education, environment, intent, and so on (Trask \& Mayblin, 2005). For example, a teacher might believe that to help children with oral language, teachers must use colorful vocabulary that is original and appropriate by supplying as many words as possible for a given object (Payne, 1996). Alternatively, Freeman and Freeman (2004) advocated that using simple sound, vocabulary, and sentence structure initially and proceeding to more complex language would benefit students more in language learning. In sum, Genesee (2000) explained that brain research has shown that the human brain needs both variations to make simple and complex circuits simultaneously activate the neural network to create automaticity in the memory. The use of one of these techniques over other techniques could result in some students retaining less information and perpetuating the discontinuity between teachers and students.

Language Structure. As stated above, students need variation in language use to maximize learning (Genesee, 2000). Berry and Kim (2008) explained the difference in language structure in terms of surface and deep language. Surface language is literally what is said and relates to surface learning in that it is what is first heard by the listener. They describe deep language structures as hard wired into long term memory. Deep language structures, similar to deep learning, are not merely what is said, but what is 
actually meant. This information is embedded in the brain and can be transferred to other situations.

Deep language learning occurs first in language acquisition. Slobin (1973) contended that children express semantic intentions before mastering syntactic or dialectic systems. Slobin's research yielded cases of bilingual children's understanding and use of two languages. He found that children who spoke more than one language would express ideas first associated with certain languages. Then children would express ideas in the language that had the simplest syntax. Eventually, this would lead to translation capabilities to express any meaning in both languages. Gonzalez (1984) also found that it is possible to form a thought or image in one language and express it in another depending on the cultural experience and language utilization. Once children have something meaningful to say, they may look to teacher examples or explicit direction of how to express those thoughts with new language forms. Conversely, children may use their native language forms to express new concepts. Multilingual teachers may also find certain instruction is better expressed and understood according to specific language experiences.

A teacher can determine teaching approaches by analyzing a student's language use. After determining a student's language use, a teacher can adjust their language use to align with that of the students. Conversely, a teacher can teach the students various ways to use language. Teachers must be able to explain why a particular sentence is good or bad so that students can understand and produce grammatically acceptable sentences (Justice, 2004). For example, when children correct themselves while speaking, it tells the teacher that those students have knowledge of the system of 
language structure (Lindfors, 1991). They can understand language at the deep structure level. Though the understanding is not as comprehensive as an adult's, listening to students speak gives teachers evidence of student's language system. This evidence can help teachers produce appropriate speech acts and determine students' comprehension levels. Hudson (2010) affirmed that the ability to compare linguistic similarities in one's own native language to the dominant language may also foster pride in speaking both languages.

A classic example that illustrates the difference between surface and deep language structure comes from an experiment performed by Labov in 1969. He asked African American youths who did not speak the standard dialect of English to repeat the sentence: "I asked Alvin if he knows how to play basketball." The boys were unable to repeat the sentence. Instead they produced the sentence in a version of nonstandard English. The most common sentence produced was: "I axt Alvin does he know how to play basketball." The sentences that were spoken by the boys displayed the same deep structure, but with different surface structure making the point that often students can understand the meaning of spoken language, but not be able to produce the surface grammar. Moreover, teachers must be careful not to equate the inability to use a certain grammatical forms with the inability to understand the concepts that underlie spoken language (Stubbs, 2002). Teachers must become more sensitive of language use in interactions with children.

Teachers sometimes complain that students know how to use and comprehend language, but are unable to talk about language (Freeman \& Freeman, 2004; Justice, 2004; Lindfors, 1991). In other words, knowing language and knowing about language 
are different. Furthermore, having common vocabulary to explain language especially within its context is an important aspects of the ICC framework (Scollon \& Scollon, 2001). In contrast, an informed teacher understands that linguistic rules are internalized by children even if they cannot label the rules or fully express them (Justice, 2004). A teacher's attentiveness to the influences of spoken discourse in a classroom, as well as the instruction given to the students, allows for exploration of how meaning is expressed in various situations for various reasons. For example, if a teacher assigns the task of writing complete sentences, but does not effectively explain what a complete sentence is or that it is dependent on its context, the teacher has missed an opportunity to help students build on the relationship between the surface and deep structure of a sentence, which gives them the knowledge to produce complete sentences (Berry \& Kim, 2008). Lindfors (1991) pointed out that teachers can recognize when the tasks the students are engaged in require the use of language or the knowledge of how language works.

Instructional Approaches. Effective teacher talk and social classroom discourse has been firmly linked to a student's choice of deep learning strategies (Berry \& Kim, 2008). Traditional modes of discourse are persistent and many teachers tend to teach the way they were taught with more surface learning strategies. Berry and Kim (2008) added that confusion in the development of learning strategies in students occurs when teacher language use does not align with instructional strategies (i.e., surface or deep). In other words, teachers must be able to combine different approaches skillfully at different times using different language structures. For example, many teachers are skilled at sequencing, practice-review, and direct questioning, but are not confident in modeling, supporting social interaction or facilitating peer collaboration (Berry \& Kim, 2008). 
Furthermore, one-sided instruction methods like didactic teaching or specific language dominance violate many learning laws and leave communicative skills underdeveloped (Blommeart, 2007). Our brains develop through cognitive and social environments. Jensen (1998) argued that student-teacher as well as student-student discussions are critical for wiring language and communication skills into the brain. He also explained that because emotions and cognition seem to simultaneously release chemicals to the brain, meaning that is full of associations and personal relevance is more fully mapped into our memories creating deep learning. Similar to helping students find strategies to access their pathways to memory, helping them recall personal associations is also important for deep learning.

A wide range of linguistic and cultural knowledge and instructional techniques must be used to inform classroom practice. Teachers with an awareness of ideological beliefs, language use, deeper inquiry into language misunderstandings, and appropriate use of various instructional methods have an advantage in a linguistically diverse classroom. Furthermore, instructional techniques that accommodate all students may require an ideological change - from "teacher as authority" teaching to "teacher as coinvestigator" teaching. However, Viiri and Saari (2006) asserted that teacher talk, such as teacher-led dialogue or teacher as facilitator dialogue, is the dominant activity in most classrooms.

\section{Coursework and Beyond: A Teacher Preparation Program Perspective}

According to Carroll (1964), knowledge and experience influence a teacher's belief systems and underlie their thinking and behavior. Further, teacher beliefs manifest in teacher talk and methods of instruction. Teacher talk influences the degree of success 
learners achieve (Lenski, Wham, \& Griffey, 1998). Additionally, Ball (2000) contended that preservice teachers enter teacher preparation programs with limited perspectives and unexamined beliefs about language use, especially with regard to students with diverse backgrounds. She further stated that the limited perspectives and engrained biases that preservice teachers bring with them into teacher education programs influence their negative evaluations of diverse student's oral language. Additionally, preservice teachers are uninformed about specific language issues faced by students in the classroom; especially issues faced by students that are not proficient in Standard English. Moreover, preservice teachers tend to feel that in teaching diverse students, cultural and linguistic problems can be solved with add-on curricula (Vavrus, 1994). Uncovering the beliefs and knowledge that preservice teachers bring with them to teacher preparation programs, curriculum can be designed to provide teachers with knowledge about how language works in diverse classrooms (Barcelos, 2003).

Despite the fact that teachers encounter students daily that understand and speak various forms of language, few teacher education programs require courses including linguistic studies which help teachers understand various aspects of language use (Moats, 1994). Additionally, only 5\% of the teachers in a national survey reported feeling prepared to enter culturally and linguistically diverse classrooms (National Center for Educational Statistics, 2003).

\section{Teacher and student disparities}

To be prepared for diverse classrooms, educators need to develop awareness that teaching practices in the United States are based on certain cultural norms and values (Gay, 2000). Gee (2001) added that the norms of the teacher and teaching practices may 
not align with those of the students. For this reason, creating ways for preservice teachers to increase their awareness of multiple perspectives and provide ways to effectively teach minority students is beneficial (Godley, Sweetland, Wheeler, Minnici, \& Carpenter, 2006).

Moreover, Ladson-Billings (1999) contended that the cultural diversity of the teaching force as well as their social and economic conditions does not reflect the student population present in most schools. She also said that many teachers reported having no training to meet the needs of the high number of ELLs. Disparate teacher and student backgrounds is not a new phenomenon; disparities have been reported since 1969 (Ladson-Billings, 1999). Ladson-Billings (1999) explained, however, that this issue has still not been addressed. Sleeter (1992) also explained that there exists a vast body of knowledge about bilingual and multicultural education, however, teacher preparation programs have neglected to inspire the epistemological and methodological transformations required to rectify the gap between teacher and student backgrounds that create many of the communication issues.

Melnick and Zeichner (1998) explained that addressing this gap between teachers and students might look different in different preparation programs such as changing recruitment and selection practices, preparation curriculum, or broader institution reform. For example, Harberman (1996) argued that the best way to train teachers to work with culturally and linguistically diverse students is to only select those candidates who already have experiences, knowledge, and dispositions that enable them to teach culturally and linguistically diverse students before they enter a teacher preparation program. He found that typically successful teachers of diverse populations are 30-50 
years old, of color, from urban areas, have children, and have learned to live normally in environments with violence. Additionally, Harberman (1996) stated these ideal candidates would be able to focus their training on more specific instructional methods.

Godley, Sweetland, Wheeler, Minnici, and Carpenter (2006) mentioned another strategy to address the discrepancy in backgrounds. They contended that teacher education grounded in linguistic research and practice might raise an awareness of issues of linguistic diversity and provide teachers with techniques, strategies, and resources for negotiating miscommunications and misunderstandings.

The Teacher Language Awareness (TLA) movement was created to increase awareness and enable teachers to speak and instruct appropriately and effectively using knowledge of the underlying systems of language (Andrews, 2007). Andrews (2007) described teachers with language awareness as understanding the complex blend of language use and language comprehension with the ability to provide the appropriate amount knowledge in the appropriate manner to the students to lessen any barriers. He further stated that language awareness acknowledges the differences in language without applying the deficit model. The knowledge required to achieve this awareness includes the properties of language, cultural influences, forms language can take, and the relationship between language and ideology (Carter, 1994).

The model of TLA depicts the characteristics of teacher language awareness (see Figure 4). It diagrams the interrelatedness of language proficiency and pedagogy as well as the knowledge needed by teachers to access language awareness. Knowledge of language systems, production, and how and when to use language, knowledge of learners, 
and knowledge of contexts, curriculum, and methods of instruction must be integrated for proficiency in language and content.

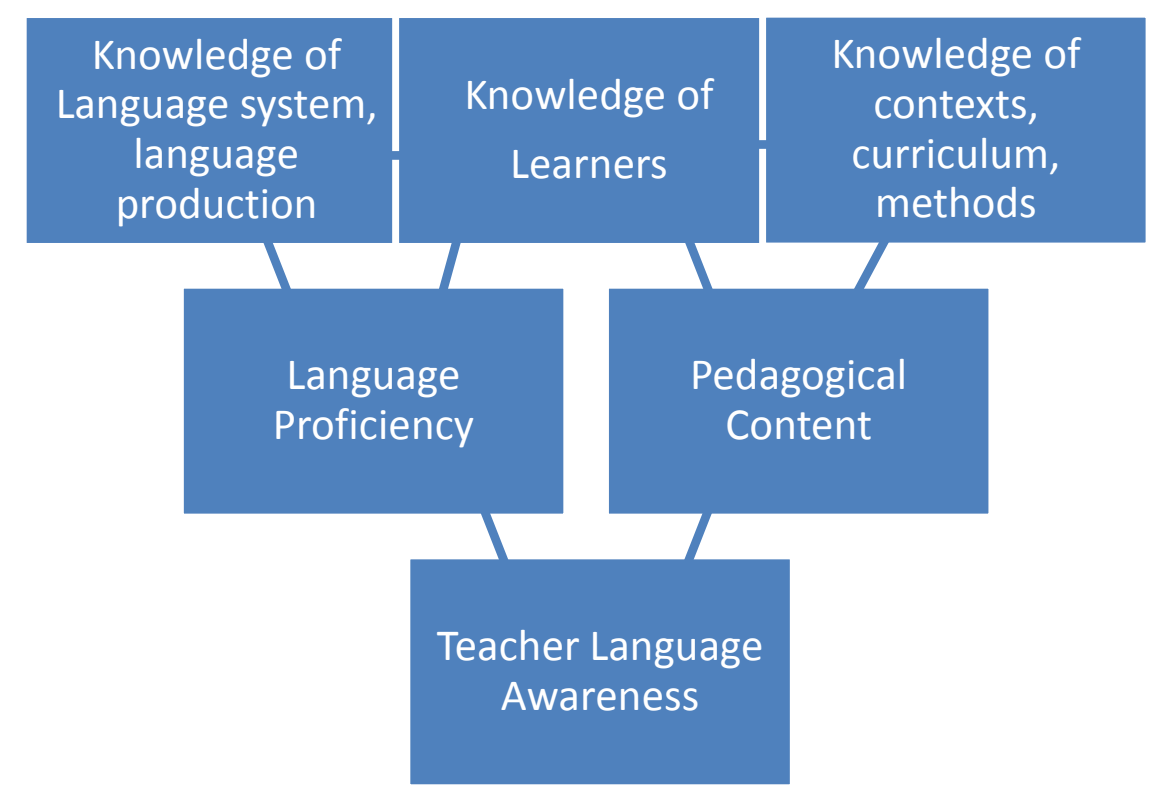

Figure 4. Model of Teacher Language Awareness. Adapted from "Model of Teacher Language Awareness”, by S. Andrews, 2007, Teacher Language Awareness, p. 31.

Ball (2000) contended that preservice teachers are not aware of language theory; therefore, this information has not made an impact on their awareness, knowledge, or practice. Further, preservice teacher knowledge is not informed by current research on linguistic diversity causing them to rely on pre-existing beliefs. Teacher preparation programs should inform preservice teachers about theories and practice related to language use, especially in diverse classrooms.

Lack of training in linguistic knowledge. Institutions of higher education have considered the question of how to best address these barriers and prepare preservice teachers by designing various models of coursework and field practice (Gay, 2000). 
Teacher preparation programs recognize the need to provide curriculum that supports training for teaching in diverse classrooms and develop a comprehensive curricular vision (Darling-Hammond \& Baratz-Snowden, 2007). However, interpretations and approaches vary considerably. Darling-Hammond and Baratz-Snowden (2007) explained that teacher preparation programs often lack the ability to connect content with contexts in which the content will be applied in a meaningful way.

Researchers found that preservice teachers described four significant barriers to implementation that their teacher's preparation programs did not address. First, Sleeter (2000) stated that preservice teachers that brought knowledge of diversity to teacher preparation programs and learned skills in their courses felt their teacher preparation programs were not designed to extend this knowledge into the classroom. Furthermore, Safford and Kelly (2010) found that teachers in their study stated that they simply did not know how to apply their linguistic knowledge in the classroom with current curriculum constraints. Finally, Smitherman and Villanueva (2000) found that linguistic courses in teacher education programs had no discernable effect on teachers' attitudes and practices because the linguistic courses may have failed to impact teachers' behavior. Yet, Cross (2003) noted many of these programs did not teach educators how to integrate the information into their classroom practices; therefore, the teachers dismissed the courses as irrelevant.

With the challenges that teachers face in classrooms today, teacher preparation programs must design broad curricula that are based in an integration of many aspects of teaching and learning which prepare preservice teachers to enter culturally and linguistically diverse classrooms. Research findings in each field of applied linguistics 
have implications for education by creating foundations to inform pedagogy and create better prepared teachers (Hornberger, 2001; Shuy, 1981; Spolsky, 1981).

\section{Summary}

In an exploration of what educators need to know, findings in articulation, psycholinguistics, neurolinguistics, sociolinguistics, and educational linguistics have implications for the field of education. Recently, the contributions of various fields within linguistics have added much to the analysis of conversational dynamics, interculturally as well as intraculturally, shaping beliefs and behavior. Spolsky (1978) advanced that these contributions require careful thought and dialogue among the fields to create solutions to issues raised by linguistic diversity. Ideally, he added, the solutions should frame diversity as a resource. He also noted that while descriptive analysis can inform language teaching and learning, a description is not a prescription.

Descriptions of language use can be helpful in understanding language pronunciation. The development of the World Englishes (Jenkins, 2009; Kachru, 2008) was instrumental in conveying the fact that there is no one prescribed and Standard English pronunciation that should be used in a classroom. Ur (1991) emphasized that the context in which language is spoken should provide the appropriate pronunciation or describe an accent that will be easily understood by all the speakers of the language. Additionally, Miller (2009) emphasized that teachers are required to understand English sounds to teach the literacy curriculum in United States classrooms. He also states that teachers must know the relationship to other language sounds in order to communicate effectively and maintain comprehensive dialogue with students. With teachers modeling 
good sound skills and students directly and indirectly learning sounds, reading and spelling as well as listening and speaking skills are enhanced (Miller, 2009).

Teachers desire pronunciation training and specifically International Phonetic Alphabet (IPA) training (Miller, 2009; Fillmore, 1985; Ur, 1991). Research indicated that an understanding of components of pronunciation such as sound discrimination and phonology is important for teachers of diverse classrooms (Freeman \& Freeman, 2004; Justice, 2004). The relationship of phonemes and allophones and voiced and voiceless substitutions help teacher talk remain purposeful and comprehensible. For example, in German, voiced consonants become devoiced at the ends of words (Obler \& Gjerlow, 1999). The word "bath" is pronounced/bat/. German speaking students hearing /ba日/ may need extralinguistic cues to understand the meaning. Conversely, the students may hear /bat/ when "bath" is said and transfer the /t/ sound to other words using / $\theta /$; always hearing $/ \mathrm{t} /$ for $/ \theta /$. Phonological experiences such as these examples can highlight various language use. Students may also lose the ability to distinguish sounds in specific languages they are exposed to as they age. Studies have shown that older Japanese students cannot differentiate /r/ and /1/. Not only does this create aural comprehension issues, these issues translate to written language as well. Miller (2009) posits that it is useful for teachers to have visual support for auditory discriminations between sounds so that the difference between /th/ $\theta$ (think) and /th/ $ठ$ (that) makes sense. A visual relation between all 44 phonemic symbols and the 44 known sounds of English can simplify the auditory discrimination process. 
The neuroscience literature describes the auditory discrimination processing of language in the brain involving not only distinction of phonemes, but also parallel processing to decipher semantic information. For example, exposure to unfamiliar speech sounds is initially registered by the brain as undifferentiated neural activity. Neural activity is diffuse, because the brain has not learned the acoustic patterns that distinguish one sound from another. As exposure continues, the listener (and the brain) learns to differentiate among different sounds and even among short sequences of sounds that correspond to words or parts of words. Neural connections that reflect this learning process are formed in the auditory (temporal) cortex of the left hemisphere for most individuals. Higher order neural circuits that are activated by contextual information associated with the word doggie can prime the lower order circuit associated with the sound doggie with the result that the word doggie can be retrieved with little direct input. Complex circuits can be activated at the same time as simple circuits, because the brain is receiving input from multiple external sources - auditory, visual, spatial, motor (Genesee, 2000). Recent research shows that the traditional ways of teaching to a specific area of the brain is no longer considered a valid technique (Genesee, 2000). Brain systems in humans interact together as a whole brain with the world through connections and associations (Elman, Bates, Johnson, Karmiloff-Smith, Parisi, \& Plunkett, 1997).

Current studies have shown that a teacher's awareness of word recognition and the relation to semantics is critical to monitoring his or her own language behavior (Marslen-Wilson and Zwitzerlood, 1989; McQueen, 2009; Moss, Tyler, \& Taylor, 2009). The use of ambiguous words and non-standard pronunciations can inhibit the student's ability for automatic word recall and word meaning. Recognition and comprehension 
rely on features of rich sounds, mapping between acoustic and articulatory phonological information, functional lexical items, words, and affixes (Pulvermuller, 2009). Phonological capacity is important when comprehending words simultaneously; however, a lack of phonological maintenance does not prohibit the integration of syntactic and semantic information (Martin, Vuong, \& Crowther, 2009). This information hinges on a teacher's articulatory behavior and sentence-level speed of speech.

Research studies have shown that knowledge in the deep and surface structure of language use is important in order for teachers to use language effectively in the classroom (Fraser, 1970; Labov, 1969; Lindfors, 1991). A teacher able to call upon his or her knowledge of language structures and semantics is more apt to create mutually intelligible classroom discourse in which conversations about varying surface structure such as, "He is knowing the answer" versus, "He knows the answer" that leads to communicative effectiveness. In alignment with the theories of communicative competence and English as a lingua franca, increasing the understanding of the deep structure of language which is the source of true semantics is the overall goal (Jenkins, 2009).

Prosody is also an important part of speech comprehension according to the literature (Obler \& Gjerlow, 1999). Suprasegmental elements of production can cause confusion cross-linguistically. English is a stress-timed language meaning that the duration is evenly spaced between stresses. French is a syllable-timed language meaning stress is on every syllable. Intonation patterns for tonal language speakers and non-tonal 
languages are very different. With the theory that language is processed (phonological systems) interdependently, prosody issues in perception or production increase. Stress patterns in words and sentence intonation between tonal and non-tonal languages can also be a source of misunderstandings.

Teachers cannot be expected to know how to speak every language their students bring into the classroom. However, as broad a range of knowledge as possible about not only language systems, but history, folklore, traditions, values, attitudes, and current sociocultural situations is necessary to help show students that the background experiences with culture and language they bring to the classroom are valued. Teachers can also understand the behavior students bring as they adjust to life in the United States.

There have been few studies that have examined teacher preparation for linguistic diversity (Rickford, Sweetland, \& Rickford, 2004). Smitherman and Villaneuva (2000) found that many language arts teachers have never taken a course in linguistics or language diversity. However, language is used in every aspect of the teaching-learning dynamic. Miller (2009) describes teacher preparation that does not include linguistic knowledge as an injustice to teachers who are expected to use and teach language and an injustice to students who struggle to learn through language.

Our educational system is heavily biased toward linguistic modes of instruction, exemplifying the need to understand the importance of language use (Gardner, 1991). With the diversity present in schools, negotiating the many complexities of language and learning is vital. According to Dell Hymes (1972), 
For language in the classroom, what we need to know goes far beyond how the grammar of English is organized as something to be taught. It has to do with the relationship between a grammar of English and the ways in which English is organized in use by teachers, by children, and by the communities from which they come; with the features of intonation, tone of voice, rhythm, style, that escape the usual grammar and enter into the essential meaning of speech; with meanings of all those means of speech to those who use them and those who hear them, not in the narrow sense of meaning, as naming objects and stating relationships, but in the fuller sense, as conveying respect or disrespect, concern or indifference, intimacy or distance, seriousness or play, etc.; with the appropriateness of one or another means of speech, or way of speaking, to one or another topic, person, situation; in short, with the relation of the structure of language to the structure of speaking (p. xiii).

In conclusion, teachers in diverse classrooms need certain types of linguistic knowledge to become culturally competent communicators. Table 2.1 summarizes the research that indicates what teachers should know to effectively use language in diverse classrooms. The content of Table 2.1 is the basis for the research study that I conducted. 


\section{CHAPTER 3}

\section{RESEARCH DESIGN AND METHODOLOGY}

\section{Purpose of Study}

The purpose of this study is to describe preservice teachers' beliefs and knowledge about language. I attempted to create a holistic description of experiences, knowledge, and philosophies of language, from the perspectives of articulation, psychology, neurology, sociology, and education. Through interviews and focus groups, participants revealed the childhood and family history that established their beliefs as well as reasons for their values, motivations, and behaviors regarding language use.

This study was informed by the conceptual framework of intercultural communicative competence theory which allowed preservice teachers' language beliefs and knowledge to be studied from multiple perspectives of communicative interaction. The primary elements of the ICC framework are 1) requisite belief - an awareness of or openness to learning about cultural and linguistic differences, 2) declarative knowledge knowledge about diverse cultures including linguistic differences, and 3) behaviors ability to listen, interpret, analyze, and apply knowledge of communication with diverse others (Bennet, 1993; King \&Baxter-Magolda, 2005; Pope \& Reynolds, 1997). These elements helped shape the instrument of this study.

The instrument used in this study also was shaped by research in linguistics and education. Research findings in the fields of linguistics and education indicate that teachers must be aware of cultural and linguistic differences to teach effectively in today's diverse classrooms (Andrews, 2007). Research has tended to focus on the effects 
of "teacher talk" on student learning by analyzing one component of language such as phonology (Snedeker \& Trueswell, 2004) or a combination of two areas such as neurology and linguistics (Ullman, 2008). However, few studies explore preservice teacher views on language use in diverse classrooms and the factors that influence their beliefs and knowledge. This study is unique because it explores the language beliefs and knowledge of preservice teachers using research in neurolinguistics in relation to the fields of applied linguistics and education. Using an instrument that integrates perspectives from these fields of applied linguistics and education in a study of preservice teachers is also unique.

Spolsky (1978) stated that instruction should be designed by first identifying a problem or need within education. Pica (1994) identified teaching and using language in the classroom as problematic. Spolsky further explained that the solution should come from analyzing the problem through theory, research, and practice in the fields of education and linguistics. This study described basic language awarenesses and knowledge needed for teachers in culturally and linguistically diverse classrooms and studied the need through ICC framework and literature in fields of education and applied linguistics. Brumfit (1997) also argued that exploration into teacher's beliefs of language use might depict a better understanding of teachers' central role as educational linguists as well as classroom teachers. From Pica's (1994) perspective of appropriate approaches to educational linguistics that emphasized the importance of multiple solutions, the investigation of this study into beliefs about language use and linguistic diversity is a 
descriptive exploration as opposed to a prescriptive solution. Therefore, the following research questions guide this investigation:

1) What language beliefs do preservice teachers have at the beginning of a teacher preparation program?

2) What linguistic knowledge do preservice teacher candidates have at the beginning of a teacher preparation program?

3) What are the similarities and differences in the beliefs and knowledge about language between monolingual and multilingual preservice teachers?

\section{Site Selection and Licensure Program Description}

The site selected for this study was an urban university in the Pacific Northwest. It is the largest teacher licensure program in the state. One of the four goals for the education at this university is for students to gain awareness of the diversity of human experience by enhancing their appreciation for and understanding of the rich complexity of human experiences through the study of differences in ethnic and cultural perspectives, class, race, gender, sexual orientation, and ability. Creswell (2008) advises that in qualitative research, researchers purposefully and intentionally select sites that can best help them to understand their central phenomenon. The selected site provided the opportunity to select a large variety of preservice teacher's as well as to investigate beliefs and knowledge about language through the ICC framework. This study focused on one program in this university in the Education Department; the Teacher Education Program (TEP). 
Within TEP, approximately half of the applicant pool is selected to participate in the program each year. The preservice teachers selected to enter the program chose one of four authorization levels: early childhood, elementary, middle level, and secondary content area specific licensure. Program applicants are selected based on a 3.0 grade point average, a skill test, a content exam, and proof of creativity, collaboration, and leadership potential through reference letters, a personal statement, and a group interview (Graduate teacher education program, n.d.).

Once applicants are admitted to TEP, they are placed in cohorts of approximately 15-30 members with one or two cohort leaders. In the licensure program, preservice teachers take classes, typical of other teacher preparation programs, in general education and specific content areas, multicultural education, literacy, technology, special education, classroom management, and reflective practice. Preservice teachers work in schools under the guidance of a cooperating teacher, and they complete course work samples to be taught in a classroom. Additionally, the preservice teachers participate in a practicum that allows for exposure to multiple student populations. Upon completion of the licensure program, candidates have the option to earn a Master's in Education degree by taking a Teacher as Researcher course and six credits of electives. At this time, program graduates face a highly competitive job market.

\section{Participants}

A qualitative inquiry is used to understand the meaning of a phenomenon from the perspective of the participants (Merriam, 1998). A convenience sampling technique was used to select participants from the TEP. The TEP students represent a group of 
preservice teachers with diverse backgrounds and varying experiences with languages and cultures yielding the best range of beliefs and knowledge for the study. Preservice teachers in a single section of the TEP that were studying to be language arts, social studies, music, or theater secondary teachers were asked to participate. Of the 24 students in the cohort, 23 volunteered to participate in this study. Sleeter (1999) explained that in previous studies, preservice teachers used to explore beliefs and attitudes have been white, monolingual samples. She added that these studies have yielded statistically similar data for the last six decades. According to Maxwell (1996), the goal of purposive sampling can be to adequately capture the heterogeneity of the population, or to establish particular comparisons to illuminate the reasons for differences between individuals. In alignment with Maxwell's goals of sampling and to assure an exploration that consisted of different sampling characteristics, participants were chosen by exhibiting the following characteristics: first, participants held preservice teacher status. It is important to understand what language beliefs and knowledge preservice teachers are bringing to the program and eventually the field. Second, participants self identified as being either monolingual or multilingual. To analyze a potential difference in language beliefs and knowledge between monolingual and multilingual candidates, an evaluation of self-proclaimed language status and actual proficiency in language was necessary. This was done with a demographics questionnaire and follow up interviews. By using these methods, a maximal variation sampling (Creswell, 2008) was established to achieve the best mix of monolingual and multilingual experiences. 


\section{Research Methodology}

The proposed research questions were explored by using a qualitative methodology. Wray and Bloomer (2006) explain that there has been a shift in research conducted in the field of linguistics from behaviorist approaches aligned with quantitative methods to methods aligned with qualitative approaches. Greene and Caracelli (1997) explain that qualitative methodology might be used to uncover the complexities of preservice teachers' language beliefs and knowledge. The best measure of exploring what preservice teachers believe and know about language and what interrelated factors influence these beliefs and knowledge is qualitative methodology. Hodson (2002) advocated for an approach to overall understandings that recognizes and promotes multiple perspectives. Denzin and Lincoln (1994) further defined qualitative research as using multiple focuses involving interpretive, naturalistic approaches to the subject matter. Qualitative methods allow researchers to study an issue in depth and without being constrained by predetermined categories (Patton, 2002). This method can produce a wealth of detailed information increasing the depth of understanding. This study structured the use of qualitative methods including responses to efficacy statements, oral histories, and interviews.

To explore the research questions, a multi-categorical instrument was developed. The instrument used for this study consisted of a demographics questionnaire and a survey that analyzed preservice teacher's knowledge and existing beliefs toward language. Survey research method is effectual for studying a variety of educational circumstances because it describes people's demographics, beliefs, and knowledge (Gay, 
2000; Picciano, 2004). A linguistic knowledge assessment was used to ascertain what degree of linguistic knowledge preservice teachers have at the beginning of the teacher preparation program. The instrument was created from a thorough investigation of the existing literature. The beliefs survey was adapted from "The Beliefs about Language Learning Inventory" (TBALLI) for teachers created by Horwitz (1988). As with other studies about beliefs (Barcelos, 2006), some of the questions had to be newly created due to the new knowledge established in the fields since TBALLI was developed. The linguistic knowledge survey also was created by synthesizing standards for teachers and students from organizations for teaching and learning. The instrument was designed to measure preservice teacher's experiences, beliefs and knowledge about language to serve as a reflective tool for teachers entering linguistically diverse classrooms and as a guide for development of instruction for teacher preparation programs. The participants were also interviewed as a way to clarify their responses on the instrument.

\section{Establishing the Validity of the Instrument}

Instrument measures must meet quality standards by showing data validity and data reliability. Validating a survey means completing several steps in building a case that is "sound: well grounded on principles or evidence; able to withstand criticism or objection, as an argument" (Friend \& Guralnik, 1960, p. 1608). A valid instrument functions dependently, accurately, and with known limits (Peterson, 1984). Shadish, Cook, and Campbell (2002) explain well-known types of validity in research: internal validity, construct validity, and external validity. They define internal validity as the degree to which alternative explanations for the obtained results can be ruled out. 
Construct validity is the degree to which constructs under investigation are measured. External validity is the degree to which inferences made on the basis of the results are consistent across variation in persons, settings, and treatment and measurement variables. An instrument's content must also be valid. Fraenkel and Wallen (2003) described four aspects that make up content-related validity including:

a. The appropriateness and comprehensiveness of the content.

b. Logically getting at the intended variable.

c. Adequate sample of items or questions to represent the content to be assessed.

d. The content and format must be consistent with the definition of the variable and the sample of subjects to be measured.

In developing validity for the instrument in this study these four concepts were used to ensure each question served the intended purpose. The research instrument designed for this study required extensive review of the literature as well as validation. To eliminate the threats to validity, the questionnaire, survey, and assessment were given to experts in the field. Experts in each specialization were asked to evaluate the questions. Experts were chosen based on their research and experiences in the fields. Each participant met the following criteria: 1) earned a PhD, 2) had a minimum of 8 years of teaching or field experience, and 3) served as directors of programs, or chairs of departments related to the fields in this study. Experts were provided with contextual information about the study. They were provided with the questions that pertain to their field of expertise and the researcher's intentions for each question. These experts were 
asked to provide feedback on the clarity, appropriateness, and the alignment with intent of the questions.

\section{Reliability}

The test-retest method was used in this study to evaluate the reliability of the instrument by determining accuracy and consistency. The instrument is determined reliable if the correlation coefficient between the two tests of the same group is strong. Additionally, Teddlie and Tashakkori (2009) contend that qualitative measures use credibility of the participants and dependability to the extent that a phenomenon can be consistently tracked by humans to determine quality. Credibility ensures that the procedures, data, and inferences are believable and true. The research for this study can be replicated by another researcher because the instrument was reliable.

According to Teddlie and Tashakkori (2009), answering two basic questions ensured that both sets of data meet quality standards: Is the research capturing/measuring what it is intended to? Is the measurement recording accurate and consistent? By defining the constructs, the phenomenon's criterion resulted in clear and quality data. With the questionnaire, survey, assessment, and the interviews, data collection reached the point of saturation.

\section{Pilot Study Results}

The demographics questionnaire, belief survey, linguistic knowledge assessment, and interview questions were also piloted with a group of preservice teachers before the study began. The pilot was performed to ensure that all aspects of the instruments were 
informed by the preservice teachers as well as the literature. The pilot study also determined the reliability and validity of the instrument. The pilot study consisted of the questionnaire, survey, and assessment that were given to a preservice teacher cohort that was not a part of the study. The questions were reorganized and given to the same cohort on another day. The process ensured accuracy and consistency of response and interpretation. Based on the input from the participants in the pilot study and careful analysis of the survey responses, the survey was revised.

The pilot study was conducted to ensure validity and reliability of the instruments. A similar population of preservice teachers was selected for the pilot study. A group of preservice teachers were given the instruments and asked to assure comprehension of each item. As a result of the pilot study, a few modifications were made to the instrument. Ten questions were reworded slightly, three questions were thrown out due to redundancy, and six questions were added. The additions were then retested for validity and reliability. A section originally in the Linguistic Content Knowledge assessment that was formatted as a Likert scale was moved to the Beliefs Survey page. Question number 11 on the demographics study was added for clarification.

\section{Research Design}

Qualitative design involves vivid descriptions of human experiences and opinions (Yin, 2000). The design of qualitative research requires researchers to select an issue, select a sample population, collect data, analyze data, and write up the findings. The research design for this study includes construction of an instrument including a demographics questionnaire, language beliefs survey, linguistic knowledge assessment, 
and individual interviews. Because of the connectedness of interdisciplinary studies, the questions in the instrument were designed to bridge the fields of language study.

A demographics questionnaire (Appendix C) was given to the participants. This questionnaire included questions about the participant's native language and other languages spoken, proficiency levels in each language, gender, age, travel, country of origin, previous courses in linguistics, and other experiences with language. This information provided data about the participant's monolingual and multilingual status. It also provided the variables for the analysis of the language survey and assessment.

Another component of the instrument was a survey that explored language beliefs (Appendix D). Language has communicative properties such as vocabulary and syntax qualities, as well as cultural implications such as identity and power elements. Therefore, it is necessary to establish the beliefs and attitudes behind the behavior of language use among different ethnic groups (Lippi-Green, 1997). It is important to understand beliefs that underlie language behavior in order to determine individuals' receptiveness to linguistic knowledge (Barcelos, 2006). A teacher's awareness of his or her beliefs about language is important for building the foundation of conscious language behavior and will affect the students' perceptions of the language used(Banks \& Banks, 1997).

The Linguistic Content Knowledge Assessment (Appendix E) was administered to understand what linguistic knowledge preservice teachers bring to their teacher preparation program. The linguistic content consisted of basic elements of speech 
production and perception from the five perspective of applied linguistics previously referred to in this study.

Lastly, a stratified sampling of 13 participants ( 7 monolingual and 6 multilingual) took part in follow-up interviews. The information presented in the interviews explored the outcomes presented in the survey and assessment to expand the understanding of language beliefs and knowledge. Interview protocols (Appendix F) followed a semistructured format and followed analysis of all previous instruments to clarify data. An interview guide was used to ensure that each participant was asked the same questions; however, the semi-structured interviews allowed for inquiry guided by the participant for deeper understanding (Marshall \& Rossman, 2011). Areas of questioning include language beliefs and linguistic knowledge from the perspective of intercultural communicative competence. Interviews allowed for a personal exchange of information that led to a deeper understanding of current and previous beliefs and knowledge about language. All interviews were recorded and transcribed. Ochs (1979) pointed out that recording and transcribing interviews are not neutral activities. Teddie and Tashakori (2009) explain, however, that when interview data, qualitatively analyzed, are integrated and member checking is confirmed with other data such as surveys and questionnaires, qualitatively analyzed, threats to inference quality decrease. Merriam (1998) also stated that qualitative studies that seek to discover and understand a phenomenon are inductive, the meaning is mediated through the researcher as an instrument, and the outcome is descriptive. 


\section{Ethical Considerations}

Before data collection began, approval of the International Review Board (IRB) was obtained. Participants were given an informed consent form and told that their participation in the study was voluntary. After receiving informed consent from the participants, the study began. During the study, I was meticulous about my commitment to confidentiality and precautions were taken to minimize the risk for the participants as subjects. For example, confidentiality was assumed by using pseudonyms instead of the names of the participants on all materials related to the study. The data were stored in a locked file cabinet after being de-identified with the pseudonyms. The pseudonym list was stored in a firewall password - protected computer. Lists were made of participants and interviews were set up while maintaining confidentiality.

Since communication styles and ways of organizing interactions can vary by individual, I took care to be aware and sensitive of potential issues during interviews that reveal personal information. The personal comfort of each participant was a priority. To alleviate any anxieties, I established a safe environment that allowed participants to ease into the study allowing them to feel comfortable, secure, and at ease enough to speak openly about their points of view. Participants had the opportunity to read and modify the transcripts from the interview. I let participants know that their views were valuable and useful.

\section{Data Collection}

Merriam (1998) stated that research data are typically collected through interviews, observations, and documents; however, researchers should use more 
methods to increase the validity of the study through triangulation. Denzin and Lincoln (1994) identified several types of triangulation. One type involved the convergence of multiple data sources. This layering of data and follow up member checking ensures that findings and interpretations are accurate, trustworthy, and credible. Merriam (1998) asserted that member checking supports the researcher's attempts to derive appropriate themes and interpretations from the data collected.

Triangulation in this study was achieved through the three components of the instrument, interviews, and member checking. The data were collected and each component was analyzed separately, then reanalysis occurred through interconnecting themes between each component of the instrument and the interviews (Creswell, 2008). Throughout the interviews, member checking was used not only to confirm survey results, but also my interpretations of the interview data.

Data collection included the demographic questionnaires, belief surveys, and linguistic assessments to evaluate what preservice teachers bring to teacher preparation programs. Table 3.1 shows the data that were collected and time frame for collection.

\begin{tabular}{|l|l|l|}
\hline \multicolumn{2}{|c|}{ Qualitative Data Collection Tools } \\
\hline Data Source & Administration & Frequency of Administration \\
\hline Questionnaire & Once & September \\
\hline Survey and Assessment & Once & September \\
\hline Interviews & Once & November/December \\
\hline
\end{tabular}

Table 3.1 Instrument and Timeline 


\section{Data Analysis}

According to Marshall and Rossman (1999), data analysis is the process of providing order, structure, and interpretation to collected data. The qualitative data were analyzed to gain further knowledge about preservice teachers' language beliefs. Teddlie and Tashakori (2009) stated that qualitative analysis is inductive and investigation of the data might "lead to themes... that are grounded in the data." Generating emergent themes from the data provided common patterns. Using the constant comparative method allowed comparisons of the different pieces of data in this study to refine categories and gain deeper meaning (Teddlie \& Tashakori, 2009).

Marshall and Rossman (2011) argued that flexibility in the interview aligned with the fundamental assumptions of a qualitative paradigm. They explained that "the participant's perspective should unfold as the participant views it, not as the researcher views it" (p. 144). For this reason, the interviews were semi-structured. A protocol for the questions was established to ensure that each participant was asked the same questions. Furthermore, to understand an emic view of the participant's perspective, other questions were allowed to evolve out of the participant's frames and structure. The interviews were audio taped and transcribed. To limit the problematic nature of transcribing, I took notes during the interviews and used transcription software (Livescribe). Also, I used the member checking method of verification.

According to Marshall and Rossman (2011), coding data is the formal representation of analytical thinking. Bogden and Bilken (2007) further stated that the coding system is the process that allows the researcher to look for patterns and 
regularities. Patterns of beliefs can emerge "from descriptive, detailed data that has been gathered unobtrusively and nonjudgmentally from a position of acceptance" (Pitman \& Maxwell, 1992, p. 760). Coding categories can be modified as analysis and reduction occurs. Krippendorff (2004) defined reducing as summarizing or simplifying data using statistical techniques or other methods. The instruments in this investigation provided a variety of data for qualitative evaluation. The questions were disaggregated into articulation, psycholinguistic, neurolinguistic, sociolinguistic, and educational linguistic questions to determine possible codes or themes for analyzing the data. Due to the integral nature of language, the questions were also separated by relational themes. Initially, the coding of the interviews was determined by the coding that occurred from the surveys. Four primary constructs were identified and provided a structure for the data analysis. However, due to the emergent and inductive nature of qualitative research, the process of open-coding was also used (Strauss \& Corbin, 1990). After rereading the interviews, other codes developed.

According to Geertz (1973), an analysis that is based in an individual's recognition of patterns of meaning and social action combined with a systemic analysis of the data with structured variables contributes to a thick description of the phenomenon that contributes to contextual and theoretical understandings. The findings of this study may enable the development of linguistic curriculum to adequately prepare preservice teachers to work in linguistically diverse classrooms. 


\section{Limitations}

There are limitations when interpreting the data from any study. This study also has limitations. First, the use of self-reporting is a limitation. Some participants may respond to what they think the researcher might want to hear or to what is academically and socially acceptable. Second, researchers bring biases into studies. My bias was partially offset by the member checks. Member checking was critical in this study because of the assumptions that were revealed by participants. As participants were asked questions, they were reflecting on their own assumptions as well as questioning the assumptions of others. I consistently asked participants to clarify their intentions to fully understand their meanings. Third, preservice teachers might have had different interpretations of the questions than I intended. However, many of these limitations were minimized by clarifying answers with follow-up interviews. A fourth limitation is that the linguistic knowledge necessary to succeed in the classroom might vary based on the students present in the classroom. Another limitation resulted from the instrument. The breadth of information required to fully understand preservice teachers demographics, beliefs, and knowledge restricted the depth of questioning about each specific topic. However, additional interview questions offset the limited number of assessment questions allowed for each section. Also, when added to the literature base, this study contributes further understandings about teachers' linguistic knowledge. The sample context creates another limiting factor. One urban university in the Pacific Northwest of the United States was the site for this research, though future research might compare preservice teachers in other contexts. The small sample size also restricts generalization to all preservice teachers. However, it should be noted that the participants represent a 
common profile with regard to gender and ethnic composition that has been mentioned in the research (Artiles, Barreto, \& Pena, 1998; Berry \& Kim, 2008; Rao, 2005; Safford \& Kelly, 2010). The fact that the participants in this study match the typical population of teachers in United States schools helps to strengthen the generalizability. 


\section{CHAPTER 4}

\section{FINDINGS AND DISCUSSION}

\section{Overview}

This study explored preservice teachers' beliefs and knowledge about language. The instrument was administered to a group of 23 preservice teachers. Follow-up interviews were conducted with 13 participants. Descriptive qualitative methodology was used to analyze the data through an ICC framework as described in Chapter 2. Findings from the data analysis are grouped together by themes that emerged. The following research questions were the basis for the study.

\section{Research Questions}

1) What language beliefs do preservice teachers have at the beginning of a teacher preparation program?

2) What linguistic knowledge do preservice teacher candidates have when entering a teacher preparation program?

3) Is there a difference in language beliefs and knowledge between monolingual teacher and multilingual preservice teachers?

\section{Demographic Questionnaire Findings}

Twenty-three of the preservice teachers from one cohort in one graduate teacher preparation program participated in this study. The participants of this study were given a Demographics Questionnaire to determine basic background information about their cultural and linguistic beliefs and knowledge. (See Appendix C.) The demographic questionnaire confirmed that there were monolingual and multilingual participants. The 
demographic survey indicated that this group had 17 teacher candidates who were monolingual and six who were multilingual.

The demographic information for the participants can be found in Table 4.1. The participants ranged from 23-38 years of age. The male to female ratio was 9/13. All of the participants were born in the United States, except one. Of the 23 participants, $68 \%$ of them reported that they travel occasionally and $6 \%$ said they travel frequently. While only one of the participants never had the opportunity to leave the United States for travel, $86 \%$ of them occasionally travel outside the United States.

Beyond the required two years of high school foreign language, 13 participants took language classes because they were required in college and seven took language classes because of personal reasons and the desire to learn. Thirteen participants continued their foreign language learning and 16 participants took various linguistics classes to learn more about how language worked in general. Thirteen of the participants claimed that they had only taken these courses because they were program requirements. Ten of the participants said they had instruction about language prosody. Twelve of the participants explained they did not have instruction about language prosody.

The participants (92\%) stated that exposure to language activities or practices was occasional prior to the teacher preparation program, but only one said that involvement in language practice was learned during professional development. Fifteen of the participants said they had learned some second language techniques either in a class or field experience. Four of the participants reported that they had no training in language 
acquisition or language practices. Participants explained that teachers should know about language when teaching in linguistically diverse classrooms. Among the most mentioned information that teachers should know about language knowledge of students was their culture context (nine participants), student's native language(s) (eight participants), language systems in general (eight participants), standard English use (six participants), and barriers to language learning (three participants). Participants' knowledge of strategies or techniques to instruct diverse populations was self-reported as minimal. Eight participants stated that they had some knowledge about differentiated instruction, four participants expressed awareness of the importance of paralinguistic cues such as visual and gestures (nonverbal), two participants shared knowledge of having the appropriate learning environment for students, five expressed knowledge of reading and writing strategies for students struggling with comprehension or other language issues, and nine participants said they had no knowledge of any strategies or techniques to instruct diverse populations.

Participants were asked about their philosophy on the best language of instruction. Fifteen of the participants said "English only" classroom instruction was the most appropriate for all students. They felt that it created "continuity," "appropriate and correct” English learning, awareness of "dominant usage," understanding of language used for taking the standardized tests, knowledge of academic and professional language, knowledge of how to "use language to their advantage" in our culture and society, understandings of "appropriate academic language in context," "clarity," "preservation of the English language," "ability to use standard English," "proficient language use," 
respect for the precedence set in academic history, and "academic skills for an academic future." Eleven of these participants specified that Standard English should be used for instruction, but support for learning English should be provided. Support, to these participants, might even mean providing alternative classrooms to meet student's individual needs if students are severely limited in English. Participants acknowledged that other registers and dialects were acceptable at other times in school. However, Participant F explained that "other dialects and languages are what they (students) learn at home." Participant V questioned whether "the use of standard English was appropriate for classroom instruction to the masses because it is standard. Is the assumption that everyone can understand language if it is a standard version?" This comment allowed the exploration of the underlying foundation for some of this preservice teacher's beliefs. Many participants (43\%) in the demographic survey initially responded that yes, "standard English is understood by more people in the world" and therefore "better for instruction." Yet, after a short reflection, some of those interviewed began to question if using standard English only would be "more readily understood" or if they believed using standard English only would "benefit students in the long-run because it is the language of power." They expressed a "fear of limiting the student's opportunities to succeed in the future."

In contrast, seven of the participants were very strongly in favor of bilingual education. According to these participants, "bilingual education teaches respect," "uses flexibility and creativity with language to encourage comprehension," "helps students learn how to see things that are different and still understand them," "allows students to 
gain a better understanding of the world and humanity," "celebrates languages," "teaches students that knowing two languages is positive and greatly beneficial," and "allows each dialect and language to be considered useful in context." Participant N explained that it also allows "students to learn and appreciate the social and cultural contexts that have created other Englishes so that they too can be knowledgeable and tolerant." Many participants (52\%) also agreed that providing explicit instruction on contextual appropriacy and variation in register and dialects were important supportive strategies for teachers to utilize. Participant M explained, "I think it's more important to express meaning effectively and communicate ideas. It is important to understand that language changes according to context, but context differences can be overcome and intentions can be understood anyway."

Of the twenty-three participants, 17 had varying proficiency levels in foreign languages. Eight of the participants spoke French at primarily novice proficiency levels, but two were advanced speakers. Spanish was spoken by nine of the participants ranging from proficiency levels novice through advanced. Hebrew, German, Polish, and Mandarin were spoken by one participant each with proficiency levels from novice and advanced. Thirteen of the participants, however, reported they speak English as their only proficient language, and explained that they had limited exposure to other languages. Therefore, 17 of the participants were classified as monolingual and six as multilingual.

The monolingual or multilingual status of the parents was varied for these participants. Fifteen of the participants' mothers were monolingual while three of those 
were somewhat familiar with other languages. Six of the participants' mothers were multilingual according to the participants. Eighteen of the participants' fathers were monolingual and four of them were multilingual. Languages spoken by the multilingual parents were some of the same languages the participants spoke; French, Spanish, Polish, Russian and Italian. When asked what the primary influence on their language accents were, 13 participants explained their birth place had the most impact on their accents, six said it was their parents' speech, and seven said it was environments other than their birth places that had the greatest influence on their present accents.

\section{Table 4.1}

Demographic Characteristics of Participants

\begin{tabular}{lll}
\hline Characteristic & Number & Percent \\
\hline Age $20-25$ & 13 & $59 \%$ \\
$26-30$ & 5 & $23 \%$ \\
$31-35$ & 2 & $9 \%$ \\
$36-40$ & 2 & $9 \%$ \\
\hline Gender & & \\
\hline Male & 9 & $41 \%$ \\
Female & 13 & $59 \%$ \\
\hline Country of Origin & & \\
\hline United States & 21 & $95 \%$ \\
Philippines & 1 & $5 \%$ \\
\hline Travel & 15 & $68 \%$ \\
\hline Occasionally & 6 & $27 \%$ \\
Frequently & & \\
\hline Opporunity leave & \\
\hline
\end{tabular}




\begin{tabular}{lll} 
Never & 1 & $4 \%$ \\
Once & 1 & $4 \%$ \\
Occasionally & 19 & $83 \%$ \\
Frequently & 1 & $4 \%$ \\
\hline Language Courses & & \\
\hline Middle school & 4 & $18 \%$ \\
High School & 22 & $100 \%$ \\
Required College & 13 & $59 \%$ \\
Non-required College & 7 & $32 \%$ \\
Language learning & 13 & $59 \%$ \\
Linguistics & 16 & $73 \%$ \\
\hline
\end{tabular}

Types of Activities/Instruction participated in related to language acquisition or practice

\begin{tabular}{lll}
\hline Professional Development & 1 & $5 \%$ \\
$2^{\text {nd }}$ Language Techniques & 15 & $68 \%$ \\
None & 4 & $18 \%$ \\
\hline
\end{tabular}

What should teachers know to teach linguistically diverse students?

\begin{tabular}{lll}
\hline Cultural Context & 9 & $41 \%$ \\
Students Language & 8 & $36 \%$ \\
Language Systems & 8 & $36 \%$ \\
Standard English & 6 & $27 \%$ \\
Barriers to Language & 3 & $14 \%$ \\
\hline
\end{tabular}

Knowledge of strategies/techniques to instruct diverse populations

\begin{tabular}{lcc}
\hline Differentiated Instruct. & 8 & $36 \%$ \\
Use paralinguistic cues & 4 & $18 \%$ \\
Appropriate Environment & 2 & $9 \%$ \\
Reading/writing strat. & 5 & $23 \%$ \\
None & 9 & $41 \%$ \\
\hline Philosophy of Language & & \\
\hline English Only & 4 & $18 \%$
\end{tabular}


Standard Eng. w/Support

Bilingual Ed.
11

7
$50 \%$

$32 \%$

Languages Spoken in addition to English and proficiency level

\begin{tabular}{|c|c|c|}
\hline French & 8 & $36 \%$ \\
\hline Novice & 5 & $63 \%$ \\
\hline Intermediate & 1 & $13 \%$ \\
\hline Advanced & 2 & $25 \%$ \\
\hline Spanish & 9 & $41 \%$ \\
\hline Novice & 3 & $33 \%$ \\
\hline Intermediate & 3 & $33 \%$ \\
\hline Advanced & 3 & $34 \%$ \\
\hline Hebrew & 1 & $5 \%$ \\
\hline Novice & 1 & $100 \%$ \\
\hline English only & 13 & $59 \%$ \\
\hline German & 1 & $5 \%$ \\
\hline Novice & 1 & $100 \%$ \\
\hline Polish & 1 & $5 \%$ \\
\hline Intermediate & 1 & $100 \%$ \\
\hline Mandarin & 1 & $5 \%$ \\
\hline Advanced & 1 & $100 \%$ \\
\hline
\end{tabular}

Parental Language Abilities

Mother

Monolingual

15

$68 \%$

Monoling. w/other

3

$14 \%$

Multilingual

6

$27 \%$

Father

Monolingual

18

$82 \%$

Multilingual

4

$18 \%$

Prosody instruction 


\begin{tabular}{lcl} 
No & 12 & $55 \%$ \\
\hline Influence on accent & & \\
\hline Birth place & 13 & $59 \%$ \\
Parents & 6 & $27 \%$ \\
Other Environments & 7 & $32 \%$
\end{tabular}

\section{Beliefs Survey Findings}

Research Question 1: What language beliefs do preservice teachers have at the beginning of a teacher preparation program?

Delpit (1995) stated, "[w]e do not really see through our eyes or hear through our ears, but through our beliefs" (p. 46). This study explored beliefs to gain an understanding of how participants "see" and "hear" diverse students to further interpret how teachers use their language accordingly. This exploration revealed four belief constructs about linguistics and education that included Language Acquisition, Language Systems, Communication, and Verbal Culture.

As discussed in Chapter 2, scholars argue that teachers choose their language use in the classroom based on their memories as students. Similarly, Peterson (2001) found that teachers usually teach the way they were taught and that their beliefs come from their own learning experiences and contact with cultures and languages other than their own. This argument holds true for the participants in this study. In the interviews, participants shared their reasons for selecting certain items on the surveys. They spoke of how they were raised and reflected on the assumptions that influenced their selections. 
Findings in language acquisition. The participants were asked about their understandings of the language acquisition process. They reported that six elements of language acquisition were important including (a) exposure to languages earlier in life, (b) the influence of the native language, (c) the influence of the environment, (d) individualization of language acquisition, (e) individual brain pathways of language acquisition, and (f) the context of language acquisition. Findings in language acquisition can be found in Table 4.2.

Exposure to languages earlier in life. Three themes regarding exposure to language early in life emerged from the data. Participants in this study shared their beliefs on topics including language acquisition as a young child, the influence of exposure to language early in life, and the extent of language acquisition as we age.

Only $35 \%$ of the participants believed that children are born with the ability to perceive the entire set of possible human sounds but eventually can only hear the sounds that are around them. The majority $(65 \%)$ of the participants agreed that children who were exposed to languages early in life might acquire proficiency in more than one language. From these participants' perspectives as revealed in interviews, their experience with other languages as children led to their acquisition of another language. Similarly, many participants $(46 \%)$ stated that they had heard "that when you speak two languages that the rest of the languages learned are easier to learn," Participant C disagreed. She explained that she could speak two languages and tried to learn a third while in France and "even in an immersion atmosphere, it was incredibly difficult." 
However, she also explained that she learned the third language eight years later as an adult.

Furthermore, none of the participants in the study believed that adolescents or adults could learn languages as easily as children, even with the use of aids such as memorization or mnemonics to remember the vocabulary and grammar rules. Despite literature refuting this claim, during the interviews, all of the participants focused on personal experiences of trying to learn a language later in life and reiterated beliefs about the difficulties of learning and using a new language as an adult. The interviewed participants agreed it was much harder to become proficient when acquiring language later in life. None of the participants believed that the difficulty in learning language later in life was the effect of inappropriate instructional style, lack of motivation, too many outside distractions in their busy lives, or the fact that older persons would need to know more language than a young child to be considered competent in communication. Rather, they believed that genetically it was not as easy as people age.

Of the participants that were interviewed, $78 \%$ believed that language learning and comprehension was easier if beginning the process as a young child. Participant $\mathrm{S}$ said, "I always heard it was better to learn languages young and I don't remember any difficulties learning my first language." Participant A explained this belief by relaying his childhood experiences as an example by stating,

I grew up in a monolingual, English-only household. No one in my immediate family or social circle was consistently exposed to languages other than standard, 
American English. Due to the lack of racial and linguistic diversity in the area where I grew up, I have had very limited exposure to other dialects of English such as Black English or southern English. This lack of exposure has made comprehension of other "Englishes" challenging and is something that I will need to consider when encountering students who speak other English dialects. I also have not been successful in learning new languages. It is difficult for me to make the sounds of other languages. I struggled in high school and as an adult.

Similar to this study, participants in other studies agreed that it is easier for young children to learn another language. Horwitz (1988) found that $88 \%$ of her participants agreed and Busch (2010) found that 77\% of her participants agreed that it is easier for young children to learn language. Additionally, Lenneberg (1967), a biological linguist, confirmed that children's brains are more flexible giving them a superior ability to learn languages.

During interviews, participants $(62 \%)$ in this study revealed that they were not familiar with current research regarding young children's language acquisition. They expressed possible reasons for believing that language acquisition is easier for young children. Some agreed that it is possible that because they do not remember the process of learning their first language or struggling with language as a child, they made the assumption that it was easy. Others elaborated that the struggles with language learning later in life could be because now they are more cognizant of the various aspects of language use. Participant $\mathrm{C}$ also explained that it is possible that "as a language learner, you might be hyperconscious of language use and analyze what is said too much. This 
can lead to the perception that it is harder to learn a language later in life, especially based on if you already believed learning and using a language was going to be harder as an adult. Young children don't overanalyze language use.” Further research into whether these possibilities play a significant role in the learning and comprehension of language should be pursued.

During the interviews, three participants $(23 \%)$ revealed an assumption that exposure to languages influences motivation to learn languages other than one's native language. They also argued that ability to negotiate language was deeply embedded in their personal experiences and though they acknowledged that everyone is different, participants contended that their experiences with language were the "normal and common experiences" shared by many. The contention that everyone who learns another language was motivated by their exposure to other languages and experiences influenced their abilities to negotiate language might lead to misinterpretations and erroneous expectations of student's abilities to learn English. This assumption might also lead teachers to produce negative perceptions of students which has implications for teacher preparation programs.

Language acquisition depends on the native language(s) of a student. The participants all agreed that language acquisition was time consuming; however, they did not agree on the amount of time to proficiency. When asked how long it would take a student to become proficient in English if one hour a day was spent learning English, nine participants (39\%) said 3-5 years, (9\%) specified 3 years, while seven (30\%) said 6-9 years. Twenty-two percent of the participants, however, reported that one could never 
become proficient in any language with only one hour of studying a day. Interviews revealed that participants believed that their responses were guided in part by their beliefs about (non)linguistic similarity issues between L1 and L2, individualism, or pedagogical differences of students and teachers. Furthermore, participants explained that it is possible that students speaking a non-linguistically similar native language may take more time to fully comprehend and use another language than speakers of a native language that is linguistically similar to one that is being learned or used. Many of their estimates of the amount of time for acquisition were predominantly based on individual characteristics, but Participant V stated that "it would make sense that a true understanding of a language that is not linguistically similar would take longer because more knowledge has to be acquired like cultural things and grammar." Interestingly, during interviews participants $(54 \%)$ in this study explained that their responses in the survey were also based on the idea that a new language can be learned and understood in a few years. Many of these participants (43\%) stated that they had heard this in high school and in their field experience. During the interviews, participants reflected on their personal experiences and remarked that their initial responses could not be true because they had not been able to achieve proficiency in 1-2 years in high school; that "it was not possible," "learners need more time." A few participants (23\%) stated that they believed that the methods used by the teacher and the structure of learning would affect the amount of years it took to learn language.

Each person has their own timeline for becoming proficient in a language and different factors that affect that proficiency. Although many participants in this study 
have different beliefs about the time it typically takes to learn a language, other studies have similar findings. In the study done by Horwitz (1988), participants stated it would take $1(46 \%)-5(43 \%)$ years to learn a language in one hour a day. The findings from Busch (2010) reveal similar results. Her participants revealed that they believed that because of their high school experience with learning a foreign language in one hour a day, proficiency should have been gained in 1-2 years. Busch (2010) also found that some participants felt that they did not achieve proficiency because they were poor language learners.

A majority of the participants in this study believed that the native language(s) spoken by the student would not only be a determining factor in the time to learn another language, but also in the degree of difficulty that the student would experience in learning English, the process that the student used, and how long it would take to acquire and use English. Garcia and Kleifgen (2010) contended that linguistically similar languages such as Spanish and English are easier to learn than non-linguistically similar languages such as Chinese and English. Some of the participants (30\%) recognized that English would be easier to learn if the native language was linguistically similar to English. Only 13\% of the participants that were interviewed reported that the native language of the student had no bearing on the difficulty to understand and speak English.

Fifty-two percent of the participants reported that English is a difficult language to learn and understand. Thirty-five percent explained that the difficulty depends on the individual student. Thirty-eight percent of those interviewed described specific difficulties with learning English such as that the English language has "too many sound 
variations and the grammar is to loose" (Participant G) or that "there are many exceptions to every rule and the language is not purely phonetic" (Participant P). According to Andersson (1998), there is no agreed upon scale for determining the difficulty of language. He explained that the concept of difficulty could be defined from many L1 perspectives including similarity of native language, syntax, semantics, pragmatics, or any other variation of perceptual differences. For example, understanding the syntax of a language usually comes first, but learning the vocabulary never ceases. Additionally, sound systems and culture are perceptually different or similar between the L1 and L2 based on individual qualities. Moreover, language is not uniformly simple or difficult which was understood by some of the participants (35\%) in this study.

In contrast to this study, Horwitz (1988) found the majority of her participants reported that English was of medium difficulty to learn. Busch (2010) found that the majority of her participants felt the language was of easy to medium difficulty to learn. Busch found that participants revealed that English was of easy to medium difficulty to learn because many features of English were similar to other languages and only grammatical features such as tenses were slightly hard to learn. Richards and Lockhart (1994) explained that many Chinese speakers perceive English to be one of the hardest languages to learn because it seems to have more grammar rules and it is an illogical language. Contrasts in beliefs are important to explore, especially when combined with the understanding that it takes years to become proficient in English. A teacher's sensitivity to these issues influences language use as well as patience with dialogue. 
According to Banks and Banks (1997), language acquisition is complicated, subtle, culture-specific, and a life-long process. Further, Ovando (1997) explained that teacher awareness of the complexities of language acquisition helps the teacher develop sensitivities to and respect for children who arrive in their classrooms speaking anything other than Standard English. Moreover, a teacher's expectation of support needed for language development and use of their own language develops through the knowledge of time and effort needed for language acquisition. Participants in this study share the belief that it is necessary to provide support for many years to allow for language acquisition to occur. They also believed that structure could be provided through teacher speech and appropriate instruction in the classroom. Many participants (69\%) interviewed expressed at least some sensitivities to newcomers in school. It was also clear from the participants in this study that they have a general understanding of the influence of a student's native language, but they lack detailed knowledge of specific linguistic systems and their influence on language acquisition and comprehension.

Language acquisition is individualized. During interviews, a majority of the participants (85\%) asserted that language acquisition was predominantly determined by individual characteristics. The participants, however, were divided about how individual characteristics influenced language acquisition. Participant $\mathrm{J}$ expressed a unique perspective with the assertion that every part of language acquisition was individualized even to the point that he believed that individual characteristics took precedence over "cultural, race and ethnic, and even socio-economic status." He asserted 
I think it's good to understand multiple cultural perspectives, but in the end we need to understand each kid and what their personal issues are. A student's ethnic and linguistic history may help point you to which haystack to find the needle in, but it doesn't get you much closer than that to meeting the needs of that student.

Participants were divided about whether reading and writing English was easier than speaking and understanding English. Responses to the survey revealed that $52 \%$ of the participants stated that they personally could read and write other languages, but not speak them. To highlight this commonly held belief, participants explained that "in traditional classrooms growing up, we were taught to read the book and write responses, but speech practice was not as emphasized" (Participant P). Many of these participants shared the experience that "it was not until I lived in a foreign country that I really practiced speaking other languages" (Participant C). Although they also believed that their experiences were average for all people, several participants also proposed that "the ease of language could be more individualized than [I] initially thought, especially in light of Gardner theories" (Participant S). All of the participants in this study reported agreement that all students remember more language and how to use it when their teachers use implicit and explicit teaching strategies and using the four aspects (listening, speaking, reading, and writing) of literacy simultaneously.

These findings are comparable with those of Horwitz (1988). Busch (2010), however, found that the majority of the participants in her study reported disagreement that reading and writing English is easier. Her participants believed that it is easier to speak a language than read and write. Scholars contended that speaking is a natural and 
innate act and reading is a more complex and artificial process that requires explicit instruction (Chomsky, 1968; Pinker, 2007). The contributions of these three studies indicate that individual characteristics might play a significant role in preference and ease of language acquisition.

Most participants $(85 \%)$ in this study felt that individual student traits determined many aspects of language acquisition such as time to acquire and comprehend, proficiency levels achieved, how language is used and what mode of language is preferable. However, 69\% of those interviewed postulated that culture influenced these individual traits.

\section{Language acquisition has more to do with environment than innateness.}

Although studies have shown that language learning is innate (Chomsky, 1968; Pinker, 2007), many participants in this study believed that environment has a greater impact in acquiring language. Chapter 2 of this study explores the degree to which scholars believe language is innate versus learned. None of the participants in this study believed that language learning is solely innate. Thirty-nine percent of the participants believe that language acquisition is a predominantly social process, explicitly and carefully learned with distinct stages. When interviewed, these participants explained that "the environment that surrounds a child and the exposure that environment provides determines how and when language is learned" (Participant P). Forty-three percent of the participants believe that language is acquired genetically and also requires explicit instruction. 
Language acquisition depends on context. Participants not only believed that the environment surrounding language learning was significant, they also believed that during language use, identifying the context, and choosing appropriate styles of communication for that context was significant. Interviews revealed that many participants $(65 \%)$ believe that the context of language learning and use is an important factor in language acquisition and comprehension. For many of the participants (52\%) in this study, the term "code-switching" was new, even if the concept of code-switching was vaguely familiar. During interviews, participants were given a formal definition of codeswitching and asked to reflect on the definition and examples from their experiences before being asked specific questions about code-switching. This new awareness might have had an impact on their thinking about language acquisition. Participants in the interviews not only spoke about students' abilities to code-switch, but also the importance of teachers to remember their language use in the classroom. Participant S stated, "I think that is my number one fear; pulling out something overly academic for them [high school students, specifically ELL students]. Not that they shouldn't be brought up to a higher level of rigor, vocabulary wise, just being in my own little academic world for a moment and forgetting what they know and don't know." She explained that she hoped that she always remembered to explain herself because she felt that "high school students would probably not ask what certain vocabulary meant because they would fear looking stupid." Participant F added that he has "been through college and graduate school and at times probably used vocabulary with teenagers that may not be a part of the high school lexicon." He expressed concern with "the ELL population not understanding his instruction." Findings in this study revealed participants concerns 
about needing more strategies to bridge the gaps in understanding between home or native language and school or academic language. In addition, concerns about how to teach code-switching were integral to these interviews.

Participant A explained that "it is complicated when listening to a new language to know how to use what you hear in another context. I agree with Judith Baker, teachers need to teach students that the language they are speaking [in the classroom] is academic, not job related lingo or colloquial.” Delpit (1995) argued that integrating experiences and the prior knowledge of students whose home culture and language are different than in the classrooms is important to helping students learn when to use different languages and registers. For example, she noted that students who come from an oral culture such as a Native American community may not be used to hearing words out of context.

Additionally, students may not be used to hearing academic language structures. Baker (2002) found modeling different language "codes" in the classroom and explicitly explaining appropriateness of various contexts was advantageous for students. Auer and Wei (2008) asserted that code-switching between contexts is a valuable tool not only for students to learn, but also for teachers to use.

Modeling code-switching for the students was also important to the participants in this study. Besides switching between registers for academic and social circumstances, many participants $(69 \%)$ believed code-switching is also valuable because "concepts in one language do not exist in other languages" (Participant Y). Participant C spoke about a conversation she had with a student in which the student refused to change the way he spoke for anyone. This preservice teacher, however, believed that "all of the codes a 
person knows make up their identity. You are not being someone else by switching. As you acquire different languages, dialects, and accents for use in different contexts, they all become a part of whom you are and where you can fit in."

In addition, all of the participants in this study expressed some level of sensitivity to language changing to suit various contexts. Participants also reported understandings about learning a language in a specific context and how that might or might not transfer to other situations. They believe there is value in all teachers having knowledge of codeswitching and using and learning language in various contexts.

Competency in language acquisition. Interviews in this study revealed another important theme in language acquisition understandings. Participants spoke of what competency in language means to them. Participants $(85 \%)$ believed that there are stages of development that everyone goes through and that these stages lead to competency in a language. For instance, Participant $\mathrm{N}$ described the "silent period" and Participant C described the stage "when you begin to dream in another language." Participants related these stages according to examples from their experiences with learning and understanding another language such as personal stages of acquisition and observing others stages of acquisition.

Cummins (1981) has stated that language development proceeds in stages. Besides the stages of preproduction, early production, speech emergence, intermediate fluency, and advanced fluency, he described stages from basic language and academic language development. He explained that within approximately two to three years, 
students understand language and use Basic Interpersonal Communication Skills (BICS). He further contended, however, that more cognitively demanding language (such as the style of language often used by teachers in classrooms or on standardized tests) requires a higher degree of proficiency, which Cummins termed Cognitive Academic Language Proficiency (CALP).

As discussed in Chapter 2 of this study, by proceeding through the stages of ICC, competence is seen in individual terms by participants in this study. However, the construct of competence must also be seen in interactional terms. Participants in this study believed that competence is not only based on rule-governed proficiency, but also on the ability to communicate functionally and interactively. All of the participants interviewed were familiar with some stages of language acquisition and varying aspects of comprehension.

Competency was defined by the participants in this study as

- "Being able to pick up on visual and tonal cues in a language."

- "I would correlate it to being able to articulate yourself in the language that you are speaking in a manner that corresponds with like your intellectual ability."

- "The level of your thinking and the ability to express it."

- "The comfort level of the speaker to communicate slowly and allow themselves the time to access words that do not come automatically."

- "Getting the meaning across however I need to do it." 
- "Competency goes beyond knowing the grammar rules and intonation, it requires knowing the inferences and connotations of a language."

Busch (2010) stated that when considering what creates language competence; the combination of grammar, vocabulary, structure, sociolinguistic and sociocultural aspects are important. Participants in this study agreed with this statement and stated that judging the type of competency would also require an understanding of the purpose of speaking. If the purpose aligns with the theory of linguistic competency, the focus might be more on the grammatical use of language. If the purpose aligns with the theory of communicative competency, meaning may be the focus of the language use. During interviews, many of the participants $(78 \%)$ stated their belief that communicative competence was valuable to achieve no matter what the purpose. They felt that "meaning trumps grammar" (Participant M). Participants also believed that vocabulary use was important in establishing comprehension. Participant M reported, "I think getting your point across is more important than the grammatical structure or knowing all of the vocabulary." Participant C expressed that a basic understanding of the other persons cultures would be beneficial in negotiating communication. Yet, Participant G clarified her thoughts by adding,

I think it depends on what your goals are. If your aim is to really have total competency of a language then I do think you need to know the linguistic elements. Like if I wanted to be academically proficient or wanted to attend school in a different language, it wouldn't be enough to just be able to communicate. But I think that most of the time for our purposes, in general 
communication; meaning is the most important thing. I also think that sometimes it is important to know the linguistic stuff because I think that how a culture's language is structured has a lot to do with how that culture perceives reality. The structure of the language helps shape how we think about things. Like in Indonesia where the language has just one tense, they have a different view of reality. To a certain point you can decipher meaning in languages without the knowledge of linguistic structure. If I just wanted to say something simple like goodbye, I could use other cues to help. But if I wanted to say something complicated or have a long conversation, it would be pretty tricky without knowing the grammar and word order and stress applications.

\section{Language acquisition uses different pathways for learning. All of the}

participants agreed that the human brain processes language by creating different pathways to memory in different areas of the brain. In the interviews, the majority of the participants (74\%) said that the pathways were established based on individual learning modalities. Additionally, $70 \%$ of the participants said that the specific language spoken did not determine how the brain processed language. Participants (70\%) also stated that our memory for language was the best when language was practiced in context or associations were made with the environment. Participant $\mathrm{R}$ stated that

Learning, pathways in brains, and stored information is only made by the child themselves not by the teacher. There is also a lot of current research that shows that when learning is meaningful it is remembered longer. It is most important to present learning in an interesting and enjoyable way. 
The participants expressed limited understandings about how the brain specifically learns, uses, and stores language, with the exception of using context and meaning. Participant S explained that she "would assume that since Gardner found that we all learn differently, our brains must do different things while learning. I don't know what those things are though. I also think that there are specific parts of our brains that do specific things. So maybe our brains do all work relatively the same." Participants expressed an understanding that "... how the brain processes language, spoken and heard, would be relevant for teachers to know" (Participant L).

Lenneberg (1967) researched biological linguistics and the relationship of the capacity for language and the peculiarities of the human brain function. His studies indicated that children use various methods to embed language into memory. He further found that these methods might have a relationship to the language spoken by the child. While participants in this study shared agreement with young children having a superior ability to learn languages, they had no specific knowledge of how the brain processes language from acquisition to memory.

Table 4.2

Language Acquisition Belief Results

Number and percentage of

1. Children participants

are born with the ability to perceive the entire set of possible human sounds and eventually can only hear the sounds they hear around them.

$8 \quad 35 \%$ do not learn languages as well as adolescents or adults because they do not have the strategies such as mnemonics to remember the vocabulary and grammar rules.

0 $0 \%$ who are exposed to more than one language early in life may acquire proficiency in more than one language.

$15 \quad 65 \%$


fully learn language by repeating words and sounds, without experiencing the objects or events.

$0 \quad 0 \%$

Number and percentage of

5. Language acquisition is participants

\begin{tabular}{lcc}
\hline acquired - innate & 0 & $0 \%$ \\
learned - explicit & 3 & $13 \%$ \\
a social process, with distinct stages, and limits. & 9 & $39 \%$ \\
genetically wired into every individual, but requires explicit instruction. & 10 & $43 \%$ \\
\hline
\end{tabular}

6. All of the following are considered language acquisition universals except:

Number and percentage of participants

There is a finite set of potentially meaning bearing sounds (vowels, consonants, tones) which can be produced by human vocal apparatus.

The set of sounds in its entirety is universal and available to all human beings without physical handicap.

$3 \quad 13 \%$

Language must be carefully taught to children in order for them to acquire a language properly.

$6 \quad 26 \%$

At some time in adolescence, the ability to acquire language with the same ease as young children atrophies or weakens.

$12 \quad 52 \%$

11. If someone spent one hour a day learning a language, how long would it take to become proficient?

\begin{tabular}{lcc}
\hline $1-2$ years & 2 & $9 \%$ \\
$3-5$ years & 9 & $39 \%$ \\
6-9 years & 7 & $30 \%$ \\
You can't learn a language in an hour a day. & 5 & $22 \%$ \\
\hline
\end{tabular}

12. It is necessary to know about English-speaking cultures to speak and understand English.

Number and percentage of participants

\begin{tabular}{lcc}
\hline True & 18 & $78 \%$ \\
False & 5 & $22 \%$ \\
\hline
\end{tabular}

Number and percentage of

15. English is participants

very difficult language to understand and speak.

$12 \quad 52 \%$

language of medium difficulty to understand and speak.

$3 \quad 13 \%$

easy to understand and speak.

$0 \quad 0 \%$

different for everyone.

$8 \quad 35 \%$

16. It is easier to read and write English than to learn how to speak or understand it.

Number and percentage of participants

\begin{tabular}{lrr}
\hline True & 12 & $52 \%$ \\
False & 11 & $48 \%$ \\
\hline
\end{tabular}

Number and percentage of

20. Students learning English participants 
experience a complex process that is fundamentally the same for each person.

undergo various processes of language acquisition depending on their native language(s).

$0 \%$

$16 \quad 70 \%$

find it easier to learn if they are native speakers of a language that is

linguistically similar; for example Spanish.

$7 \quad 30 \%$

can all use the same language program to learn English regardless of other

languages spoken.
Number and percentage of participants

22. Our memory for language is best when

\begin{tabular}{lcc}
\hline language learning is connected to languages already known. & 2 & $9 \%$ \\
associations are made with the environment while learning. & 7 & $30 \%$ \\
vocabulary is repeated constantly. & 1 & $4 \%$ \\
language is practiced in context. & 16 & $70 \%$ \\
\hline 28. Students remember more information when they use a process that & Number and percentage of \\
involves implicit and explicit learning. & participants \\
\hline Strongly Agree & 6 & $26 \%$ \\
Agree & 13 & $57 \%$ \\
Neither agree or disagree & 1 & $4 \%$ \\
Disagree & 0 & $0 \%$ \\
Strongly disagree & 0 & $0 \%$ \\
\hline $33 . \quad$ The human brain processes language by creating different pathways to & Number and percentage of \\
memory in different areas of the brain. & participants \\
\hline Strongly Agree & 3 & $13 \%$ \\
Agree & 17 & $74 \%$ \\
Neither agree or disagree & 0 & $0 \%$ \\
Disagree & 0 & $0 \%$ \\
Strongly disagree & 0 & $0 \%$ \\
\hline
\end{tabular}

Findings in language systems. Findings concerning preservice teacher beliefs about language systems involved not only the lexicon, but creation of shared meaning. Language systems are comprised of interrelated linguistic units such as accent, prosody, syntax, morphology, phonemes, phonology, syllabicity, tense, inference markers, variation patterns, and concept systems. (See Table 4.3.) 
All of the responses from the participants in this study revealed that the relationship language systems have with creating meaning is very important to them. Fifty-seven percent of the participants believe that language is a system of meaning shared by people. Some of the participants $(30 \%)$ also reported that language was an expressive system of communication that creates mutual understanding.

Participants were asked to imagine a scenario where the teacher uses one language system and the student uses another. In this hypothetical situation, the student has a hard time understanding the teacher and responds inappropriately creating misunderstandings. Participants were asked to explain how they would handle the misunderstandings. Many participants (62\%) stated they would enter into a dialogue asking the student to "repeat what they said" or "explain what he/she meant." Then, participants were reminded of their response to question 10. When asked about specific language miscues during reading that occur in classrooms, most of these participants $(83 \%)$ stated that they would ask the students questions to find out why the miscue occurred. Specifically, $57 \%$ of the participants reported when reading with a student, if a student miscued, the participant would inquire about meaning because different miscues mean different things. The responses to interview questions and the surveys were similar. Further, more specific situations were explored in the interviews and again participants described circumstances when misunderstandings might occur and revealed that their concern was primarily with the comprehension. They explained that aspects of language such as accent, pronunciation, and grammar could all be negotiated.

Additionally, $26 \%$ of the participants reported that the most important thing was to ask 
the student about the underlying meaning. "As long as the student obtains at least a basic level of comprehension, mispronunciation or one word miscues are unimportant," explained Participant G. Participant J explained that "the structure, like rules, of a language and the sounds and the cultural concepts in a language are only important if speech without them can't be understood.” Participants in this study expressed sensitivities to misunderstandings and explained the best way to create understanding is to ask students what they are thinking and tell students about their intended meaning. Participant P explained that "I wish my teachers would have asked me questions to find out why I didn't understand. I probably couldn't have explained exactly, but they would have been trained to analyze that and help me. I understood more than they thought. I just couldn't respond correctly." They explained that dialoguing was a good strategy for negotiating language.

Ninety-one percent of the participants agreed that all languages change slightly over time. Moreover, in the interviews, the participants reported that the systems of language use are guided by these changes. It is reasonable to suggest that the combination of language systems present in a classroom and the changes that occur will require teachers to be flexible with their language use. When asked how language changed, the most reported response was that vocabulary changed meaning which they said would not change the system level much.

As Ovando (1997) stated, educators need to understand the differences in language systems, because even differences between Standard English and Black English language systems is more than simply differences in phonology or lexicon. He further 
explained that though some educators believe that learning one's first language is simple, examination proves that communicative competence in even our first language system is a complex process that takes years to accomplish. Findings indicated participants had an understanding that language systems are complex and of the commitment of time involved to navigate different systems. However, participants lacked specific knowledge of the complexities of language systems. Aside from vocabulary and accent, however, the dominant belief among participants was that language systems were all similar. The implications will be discussed in the next chapter.

\section{Table 4.3}

\section{Language Systems Beliefs}

\begin{tabular}{|c|c|c|}
\hline 4 As acquired by each succeeding generation, language & \multicolumn{2}{|c|}{$\begin{array}{l}\text { Number and Percentage of } \\
\text { Participants }\end{array}$} \\
\hline becomes more rigid and precise. & 0 & $0 \%$ \\
\hline requires more study to use well. & 1 & $4 \%$ \\
\hline changes slightly. & 21 & $91 \%$ \\
\hline loses outside influences. & 1 & $4 \%$ \\
\hline 7 Language is & \multicolumn{2}{|c|}{$\begin{array}{l}\text { Number and Percentage of } \\
\text { Participants }\end{array}$} \\
\hline a system of meaning shared by people. & 13 & $57 \%$ \\
\hline a system to organize the world around us. & 1 & $4 \%$ \\
\hline a representation of the culture in which it is used. & 2 & $9 \%$ \\
\hline an expressive system of communication & 7 & $30 \%$ \\
\hline $\begin{array}{l}10 \text { A student in your class is taking an oral reading test. He reads "tink" } \\
\text { for the word "think." You, as the teacher, }\end{array}$ & \multicolumn{2}{|c|}{$\begin{array}{l}\text { Number and Percentage of } \\
\text { Participants }\end{array}$} \\
\hline mark the word wrong because clearly he has misread the word. & 0 & $0 \%$ \\
\hline ask the student to repeat what he has read to determine if it is a miscue. & 6 & $26 \%$ \\
\hline $\begin{array}{l}\text { ask the student what the sentence means. Phonic and graphophonics } \\
\text { miscues mean different things. }\end{array}$ & 13 & $57 \%$ \\
\hline $\begin{array}{l}\text { let the student keep reading and ignore the miscue. One word does not } \\
\text { matter. }\end{array}$ & 4 & $17 \%$ \\
\hline
\end{tabular}


Findings in communication. Themes about language as communication emerged from the data. Findings included the importance of comprehension, a teacher's responsibility in classroom communication, the importance of pronunciation, student reception of teacher speech, use of dialects in the classroom, and the relationship between communication and identity (see Table 4.4).

The importance of comprehension in communication. All 23 of the preservice teachers in this study believe that the most important aspect of communication is mutual comprehension, though there were differences of opinion as to how understandings are achieved. Forty-three percent of the participants reported that language is a communication system governed by mutual comprehension. Fifty-seven percent reported that grammatical rules and mutual comprehension equally guided communication systems. Participants that believed it was important for their development into competent English language users said that "grammar and comprehension are hand in hand" (Participant R).

Twelve of the participants (52\%) in this study agreed that when teachers and students engage in the negotiation of language, they both gain a better understanding of the language. Ten of the participants $(43 \%)$ reported that through negotiation, the teacher and student become aware of individual style differences in language as well. Busch (2010) stated that an appreciation of the complexities of communication develops when one has to negotiate with a language which is unfamiliar to them. Moreover, as stated in the literature review about ICC, to negotiate language requires two crucial traits: 1) the belief that the effort put forth will result in successful relationships and positive 
understandings, and 2) knowledge of ways to accomplish mutual comprehension. The ability to understand different communication systems and differing components of language is crucial in creating strategies that lead to mutual comprehension. Participants in this study strongly desired to create mutual meaning through communication. Their responses suggested an understanding of each of these ICC traits. Participant C explained the importance of communication as a means "to understand and seek to be understood."

In interviews, several participants (54\%) stated that while growing up, their families had emphasized "proper English that followed strict grammar rules" and that there was one "correct way to speak." Participant N explained, "Using the grammatical structure of language correctly is important." They also stated that they might not have been able to achieve good grades in school or progress in career choices without this basic understanding of the syntax of language. Participant V explained that " $[\mathrm{t}]$ here are also times when the lack of grammar knowledge may contribute to the listener's inability to comprehend." This evidence suggests a possible link between how the participants were raised and their current beliefs about the degree to which grammar determines comprehension. Participant Y stated,

Grammar is important and dependent upon whom you are addressing. I think we need to reconsider the importance of grammar in this country. Since language is constantly changing, is it necessary to have only one, singular interpretation of appropriate grammar? Should it be only the grammar used by the dominant 
culture that possesses the best education? Is grammar then being used for exclusionary purposes?

The $43 \%$ of participants that argued that comprehension was possible without grammar knowledge stated that language provides many cueing systems that can be used instead of grammar. Citing examples of travel and study in Mexico, Thailand, France, and Korea, participants described experiences that ended in an appreciation of the effort to create shared meaning. Moreover, Participant N said, "[m]aybe because much of my experience has been teaching English in foreign countries, it is much more important that children are understood, rather than completely grammatically perfect." Agreement among the participants about the complexities of communication and the deep understandings created by negotiation between languages was reported to be the underlying reason that $74 \%$ of the participants in this study reported that there is not one crucial aspect to gaining comprehension of a speaker.

There was a higher percentage of participants in this study who believed that grammar is an important component in the comprehension of language than in the studies conducted by Horwitz (1988) and Busch (2010). One possible explanation could be a consequence of whether participants were raised with an emphasis on "correct" grammar or not. Yet, the majority of the participants believed that some form of communication was still possible without complete knowledge of the syntactic structure or the culture of a language as stated in a previous section of this paper. They reported that communication in school required knowledge of syntactical structures, vocabulary, culture, and strategies. 
This study revealed strong beliefs in communication as a tool that creates mutual comprehension. Though participants revealed empathy for and the importance of negotiation of language "by any means necessary," there was no indication of a specific, systemic plan for putting this belief into practice. Furthermore, findings did not indicate specific components of language use that would be effective with diverse populations. Often mentioned was the need for instruction in practical application techniques for implementation in the classroom.

Findings in this study affirmed that though some participants (8\%) lacked significant experiences with cross-cultural communication, most of them (86\%) still had the understanding that positive belief systems about negotiating differences and strategies for creating mutual comprehension were important. However, it is unclear whether their personal experiences with grammar and vocabulary will help or hinder their decisions about language use in the classroom.

Teacher responsibility in communication in the classroom. On one hand, $48 \%$ of the participants felt that it is the teacher's responsibility to teach and use Standard English to ensure that students can use the most widely understood language in the United States. On the other hand, $26 \%$ of the participants felt it is the teacher's responsibility to teach and use many dialects and registers to help students negotiate more language. Only $9 \%$ of the participants stated that teaching students the versions of English that would help them to stay bonded with their communities was important. Yet, all 23 participants agreed that a teacher's primary instructional focus should be on ensuring that all students comprehend what is being said no matter what language needs 
to be used. Participant A further explained that he would have to use Standard English in his classroom because he does not speak another language or dialect.

Every decision a teacher makes regarding language use affects the learning potential in the classroom. Language use is guided by and guides the pedagogy selected for instruction which influences how students understand. The literature describing a teacher's role in the classroom related two common views about language use. One view describes the necessity of using only the dominant language in the classroom to provide students the tools to navigate the constructively in society (Delpit, 1995). The other view describes the importance of code-switching and the ability to navigate successfully in many communities that rely on different language and dialects (Baker, 2002).

Participants in this study reflect these two views, though most of them express beliefs in a teacher using Standard English. Monolingual participants that describe their limits of using only one language in the classroom must have strategies to compensate. These circumstances have implications for teacher preparation programs.

The importance of pronunciation. Participants had differing answers about the importance of pronunciation. In survey question 14, few participants (13\%) reported pronunciation should be the focus of language use. However, in question 13, when asked specifically about the teacher's pronunciation of English, participants (57\%) expressed beliefs in the precision of pronunciation with $22 \%$ of those participants reporting that teachers should speak with correct pronunciation or not interact with students directly. Thirty-five percent of the participants reported that it is important for the teacher to speak academic English with perfect pronunciation, while $30 \%$ of the participants felt that using 
the local dialect of English pronunciation was beneficial. Only $13 \%$ of the participants reported that varying pronunciations should be used in the classroom to allow students experience with other pronunciations of English vocabulary.

When asked about the focus of a teacher's spoken language, the majority of the participants (57\%) reported that pronunciation, grammar, and accent use were all equally important. During interviews, these convictions were even more prevalent. Participants all felt that the pronunciation used in the classroom by the teacher was important though in different ways. Participants in the interviews explained that personal experiences influenced their beliefs about teacher talk. Many of the participants (70\%) reported that when they were young, their parents emphasized not only using "correct grammar," but also "correct pronunciation" when speaking. Participants (62\%) did not believe that the focus of language used by the teacher should be on pronunciation unless they were asked specifically about pronunciation of Standard English. Similar to this study, Horwitz (1988) found that $40 \%$ of the participants believed that speakers should use correct pronunciation. However, $73 \%$ of the participants disagreed with the statement that you shouldn't say anything until you can say it correctly.

Student reception of teacher speech. Twenty-six percent of the participants believed that a focus on vocabulary would be beneficial. Participant P explained that when she was the student, having a large vocabulary contributed the most and "having the teacher use common vocabulary increased my chances of understanding." Participant B explained how hard it was for him to understand professors with "thick" accents, but that it was a good experience for him to hear vocabulary pronounced in other ways. 
When considering crucial aspects of teacher speech, the findings of this study can be compared to other studies. In a study conducted by Richards and Lockhart (1994), $42 \%$ of their participants reported that vocabulary was the most important aspect of student reception. These participants explained that when vocabulary knowledge was greater, comprehension of speech was increased. Most of the participants (57\%) in this study, however, agreed that vocabulary is important, but that "every part of language matters, comprehension cannot occur without good word choice, good grammar, and good sounds" (Participant N). Horwitz (1988) and Busch (2010) reported finding similar results. Participants in these studies reported that language learning and understanding is not just about vocabulary or grammar. A focusing on a certain aspect of language is not a belief held by most of the participants in any of these studies.

Table 4.4

Communication Beliefs

\begin{tabular}{|c|c|c|}
\hline 3. Language is primarily a communication system governed by & \multicolumn{2}{|c|}{$\begin{array}{l}\text { Number and Percentage of } \\
\text { Participants }\end{array}$} \\
\hline grammatical rules & 0 & $0 \%$ \\
\hline mutual comprehension & 10 & $43 \%$ \\
\hline Neither & 0 & $0 \%$ \\
\hline both $a$ and $b$ & 13 & $57 \%$ \\
\hline 9. In the US, it is a teacher's responsibility to & \multicolumn{2}{|c|}{$\begin{array}{c}\text { Number and Percentage of } \\
\text { Participants }\end{array}$} \\
\hline $\begin{array}{l}\text { teach only in standard English in order to insure that students use the } \\
\text { most widely understood language in the United States. }\end{array}$ & 11 & $48 \%$ \\
\hline $\begin{array}{l}\text { teach many dialects and registers of English in order help students } \\
\text { negotiate more languages. }\end{array}$ & 6 & $26 \%$ \\
\hline $\begin{array}{l}\text { teach the students version of English to help keep students bonded } \\
\text { with their communities. }\end{array}$ & 2 & $9 \%$ \\
\hline teach the "World Englishes" that exist in the classroom. & 4 & $17 \%$ \\
\hline 13. As a teacher, it is important to speak English & \multicolumn{2}{|c|}{$\begin{array}{l}\text { Number and Percentage of } \\
\text { Participants }\end{array}$} \\
\hline
\end{tabular}


using academic English with perfect pronunciation.

$8 \quad 35 \%$

using the local dialect of English pronunciation.

$7 \quad 30 \%$

correctly or not teach students directly.

$5 \quad 22 \%$

with varying pronunciations to allow students experiences in World

Englishes.

$3 \quad 13 \%$

14. During speaking, teachers should pay the most attention to their

Number and Percentage of pronunciation. Participants

grammar.

$3 \quad 13 \%$

vocabulary.

$14 \%$

all are equally important.

$6 \quad 26 \%$

18. The most important aspect of comprehending a speaker is to

$13 \quad 57 \%$ know

Number and Percentage of the vocabulary. Participants

the syntax

$4 \quad 17 \%$

the accent

$29 \%$

not just one

$0 \quad 0 \%$

not just one of these things.

$17 \quad 74 \%$

Number and Percentage of

19. When two people engage in the negotiation of language, Participants

they gain better comprehension of the language.

$1252 \%$

they acquire the language.

$14 \%$

they experience frustration that interferes with language usage.

$0 \quad 0 \%$

they become aware of individual style differences of language.

$10 \quad 43 \%$

Number and Percentage of

25. A teacher's primary instructional focus should be on Participants supporting multilingual students.

$0 \quad 0 \%$

teaching curriculum at the level of the benchmark students.

$0 \quad 0 \%$

ensuring comprehension of all students.

$23 \quad 100 \%$

an approach that challenges higher achieving students and reviews

for struggling students

$0 \quad 0 \%$

31. In a school that has primarily native Spanish speakers, Spanish should be taught as well as English.

Number and Percentage of

Strongly agree Participants

Agree

$4 \quad 17 \%$

Neither agree or disagree

$15 \quad 65 \%$

Disagree

$14 \%$

$0 \quad 0 \%$

Strongly disagree

$0 \quad 0 \%$ 
Findings in verbal culture. Verbal culture in this study is conceptualized as the direct influence that culture has on oral language use. The findings in this section include beliefs about: multiple languages and dialects spoken in the classroom and in society, multiple languages creating negative or positive effects for all students, and language as culture, culture as language. (See Table 4.5)

Multiple languages and dialects spoken in the classroom and in society. All of the participants in this study agreed that the exposure and knowledge of multiple languages is advantageous in general, but most (69\%) felt that using multiple languages in the classroom was problematic. These participants reported that a teacher that spoke many dialects could use that knowledge to communicate with students to achieve mutual understanding, but not necessarily during instruction. Participant P said, “if I spoke my student's native language, I would use that to explain everything that was not understood." Participant B, however, expressed uncertainty as to whether and how dialects should be used in the classroom. Participant N who speaks Mandarin and taught in China clarified that "only the regional dialects of a language should be allowed in the classroom. Local dialects that are not spoken by many people or widely accepted should not be allowed. Too many languages and dialects make the room too complex."

When asked about what language should be used in classrooms, $57 \%$ of the participants said that using a single Standard dialect of English would deprive speakers and listeners of language resources. However, 39\% of the participants reported that requiring a single Standard dialect of English to be spoken in the classroom was beneficial. Seventeen percent of the participants said that a single Standard dialect would 
better support student achievement and language competence. Thirteen percent of the participants said that requiring a single Standard dialect was merely encouraging the correct way to speak. And $9 \%$ of the participants agreed that classrooms requiring a single dialect teach more complex, logical, and expressive ways to speak. Participant F explained that "English is the language of power in this country and acknowledged worldwide." Furthermore, Participant A stated that "using the most commonly used language would be advantageous for students because it helps them become more proficient in the language. People who have better command of the language are more in demand and in this country; more respected." Consistent with this reporting was the 9\% of participants that also reported that Black English should be discouraged in public school settings.

Additionally, $30 \%$ of the participants agreed that a teacher should correct a student's use of nonstandard English. While only 17\% disagreed with correcting a student's nonstandard English, 43\% of the participants remained neutral to correction citing that it depended on the situation. These findings are consistent with Horwitz' study (1988) that found that participants believed that allowing students to use and hear mistakes in the language might embed those mistakes in their learning and 57\% of her participants said that allowing mistakes would make it hard to speak correctly later. Busch (2010) also found many participants thought that error correction was important. Several participants in her study cited their high school experiences when they were corrected in their language use and tested on correct forms as major contributing factors in their beliefs. 
Participants in this study reported that use of nonstandard dialects and other languages were more beneficial in society as a whole than in the classroom. Moreover, 91\% of the participants noted that Black English dialect conveys meaning for experienced speakers and listener, is acceptable as a viable communication mode in society, and carries complex information across a range of cultural groups. When asked if the elimination of nonstandard dialects of English creates social stability, $69 \%$ of the participants reported that the elimination would not create stability.

During interviews, participants in this study expressed beliefs consistent with the idea that teachers often use Standard English as a threat against failure or as motivation for success (Lippi-Green, 1997). This is evidenced by comments about the importance of teachers using only Standard English because it is the language of power and respect worldwide. Participant F explained that “we disadvantage our students if we don't teach them proper English. They already know how to speak their native language.” Even those participants that stated that they would use other languages and dialects to explain difficult content revealed that they would "correct a student's incorrect use of language because students will need to practice the correct use. Students will need to graduate high school by demonstrating proficiency in language for standardized tests and they will need to get jobs to support their families." Participant M explained that she had a conversation with a student in her field placement recently in which she had to explain to him that he "would not pass the SAT if he wrote with incorrect English grammar." These comments also confirm the assumptions these participants hold about their own values of 
language. This has implications for teacher preparation programs regarding the similarity and difference of cultural and individual values and purposes of language use.

During interviews, participants in this study revealed experiences with people who made assumptions about people's knowledge levels and interests based on the persons' culture. For example, Participant $\mathrm{C}$ explained her belief that people expected that members of differing cultures would not have the same knowledge. She made comments such as 'thinking that someone like you wouldn't know about that; our views cannot be the same because we are from different places; or you can't be articulate or discerning because you're different." She also explained that when one such conversation led to assumptions she had in another country, "the other person was just as shocked as I was that we had the same knowledge of world issues." This evidence demonstrates that some of the participants were meta-cognitive about cross-cultural experiences. Meta-cognition is one component of ICC (Scollon \& Scollon, 2001). They explained that developing components of ICC requires reflection on language use and knowledge which allows one to pay attention not only to what we say, how we say it, and how we interpret the discourse leading to more effective cross-cultural communication.

Participants (78\%) generally viewed people who speak many languages as intelligent people. Of these participants interviewed, the general consensus was that most people

that speak three or four different languages are pretty smart because it is really hard for them to learn all of those languages given the typical circumstances of 
living in the United States (Participant A), being born into a monolingual household, limited opportunities to learn and use a specific language daily and in school, and only being required to study two years of a foreign language to graduate from high school (Participant M).

Further, Participant A explained that this view of multilingualism was based on Western-centric assumptions. He stated that he had

immediately assumed that someone who live here [United States] and spoke multiple languages was intelligent. It has been my experience that would be a pretty intelligent person. But then [I thought], that there are a lot of places in the world where you are going to be surrounded by three languages at a time. So you probably wouldn't necessarily be unusually intelligent if that is what you have been surrounded by all your life.

Participant M said that she considers multilingual people to be intelligent because [she] chose not to define intelligence in a specific way, but as a form of code-switching. She explained that the ability to code-switch is not just for academic purposes and "it is an advantage that can be used in all contexts and therefore, creates a form of intelligence." She further explained that the ability to know when to speak what dialect or register equates with intelligence. However, $14 \%$ of participants chose not to answer the question about if people who speak multiple languages are intelligent. During the interview it was revealed that these participants did not think that someone who had the 
ability to speak multiple languages was necessarily intelligent. Participant D explained that

speaking multiple languages means that you speak more languages and you have the advantages of speaking more languages, but it doesn't mean that you are a more intelligent person. I would hope that it would, but not necessarily. I think you are at a real advantage if you speak multiple languages because you have that much more ammunition for expression or communication.

The majority of participants, however, in the Horwitz (1988) and Busch (2010) studies did not believe that multilingual people were intelligent or unintelligent. All of the participants that did not answer question 17 in this study believed similarly. Additionally, only one participant in this study stated that people can only truly be proficient in one language.

Multiple languages in the classroom: negative or positive effect. While previous studies reported a low number of the participants believing that English only classrooms were more effective, this study found a higher number of participants (39\%) advocating for English only. On the other hand, a little over half of the participants (61\%) reported that teachers should use the languages of the students in the classroom and teach them when each of them is appropriate. The participants in this study reflect the debate in the literature to a certain extent.

As described by the literature, commonly asked questions among educators include: should language varieties be allowed in the classroom and do they benefit 
speakers? In 1957, a study done by Abraham found that 15 out of 29 teachers believed that teachers should speak English only in the classroom. He also found that teachers (18/29) also believed that bi-lingual students should speak English only in the classroom. Ovando (1997) stated that some educators today interpret the use of anything other than Standard English in the school as a waste of educational effort, citing that English is the dominant language and should be the only language taught to allow students a chance at success in the world. On the other side of the issue, linguists and neuroscientists have pointed out that allowing native dialects and languages into the classroom is beneficial for many reasons as discussed in this paper. Participant Y explained that his school experience was enriched by his teachers allowing his dialect in the classroom. He said that he learned more in the classes where teachers understood his language and cultural struggles and that allowed him to use his language to teach others and gain understanding. He said he learned more language this way and he felt that other students gained respect for him.

Fifty-seven percent of the participants reported disagreement with the statement, "academic success will fall if teachers allow Black English to be spoken in school." Twenty-six percent of participants neither agreed nor disagreed that academic success with fall is Black English is allowed but one participant reported agreement that academic success will fall by adding that Black English because it is "grammatically incorrect."

The majority of the participants $(83 \%)$ also agreed that the monolingual/monocultural policies implemented in schools had a negative effect on 
student learning. On the other hand, $17 \%$ of participants said that monolingual and monocultural policies in schools benefited students by teaching the language and culture of power. Half of the participants (48\%) reported these policies created student failure through the exclusion of multiple perspectives. Thirty-five percent of the participants felt that these policies divided students further due to the lack of acknowledgment of difference.

Although none of the participants directly spoke of Standard English only philosophies creating situations of marginalization or instances of devaluing specific populations, many participants (69\%) expressed concern about the effects of using multiple languages in the classroom.

Language as culture and culture informs language. The majority of the participants (78\%) added that understanding the culture of the language was an important factor in gaining speaking proficiency with true comprehension. Participant Y explained that

it is both important and necessary to help students understand the American culture when helping them learn English. If a teacher is speaking English, she must also "speak" the culture because language is a part of the culture. Language cannot exist by itself. You need to know why people say idioms like "drives me nuts" and "three dollar bill." To learn that, one has to learn about the culture. 
Furthermore, Participant S expressed the importance of "exposing our students to the traditions in the American culture to provide at least some familiarity with themes and genres for understanding reading instruction. Language and culture are interrelated."

Horwitz found that a smaller number of her participants (41\%) agreed that it was important to know the culture of the language in order to speak and understand it. She also found that an equal amount of participants were unsure of the importance of the role of culture in speaking and understanding the language citing that translating languages might suffice. Conversely, Busch found that $80 \%$ disagreed that it was necessary to know the culture. Three participants in her study stated that it was helpful, but not necessary. One teacher said that people learn languages all the time without experiencing the cultures. Another stated that living in the culture may increase possibilities to practice, but also may not. Results from this study indicate that participants seem to have a better understanding of what is involved in understanding a language than participants in other studies. The literature in linguistics is divided about this issue, however, Agar (1994) shared that "[y]ou can master grammar and the dictionary, but without culture you won't communicate" (p. 29).

Participant's reflections on culture and language. Interviews revealed participants' experiences of encountering teachers that reflect these opposing views about the relationship between cultures and language. Participant A described working with a teacher that made comments such as "I have known a few Blacks so I know they like direct language." This participant described the same teacher explaining about a student that "she's Asian, therefore, she speaks broken English and can't pronounce words with 
"L's." Participant V recalled a conversation with a teacher that had always "assumed that all cultures privileged individualism in the same way as the American culture." Many participants reported experiences with teachers that generalized cultural characteristics to all of the people in a group based on experiences with few. The fact that these experiences had happened recently was disturbing to these participants. Participant G stated that these assumptions "take many years to unravel." Participant V reported that trying to "ask individuals about their cultural beliefs instead of making the assumption that an individual person can speak to the cultural opinions of all people in their race or ethnicity" is more beneficial for everyone. Yet, Participant $\mathrm{N}$ argued that "with regard to cultural and language diversity, seeking a balanced approach to teaching will be complex and advantageous."

During interviews, participants disclosed feeling that many teachers think that understanding every student's language and background is unnecessary and only complicates instruction. Participants assumed this subconscious belief was "symptomatic" of growing up speaking English in United States schools. Yet, LadsonBillings (1995) emphasized using student's native language as a vehicle for learning. Findings confirm similar sentiment to Ladson-Billings and further revealed concern for the best way to use language and cultural information besides having "multicultural day." With classrooms exemplifying cultural and linguistic diversity a resource for all, the rich experiences that exist in the classroom allow teachers to build positive environments (Nocon \& Cole, 2009). Nocon and Cole (2009) further explained that when teachers 
ignore these important experiential backgrounds, individual students can become

devalued and stigmatized.

Table 4.5

Verbal Culture

2. Classrooms that require a single Standard dialect

Number and Percentage of are merely encouraging the correct way to speak. Participants teach more complex, logical, and expressive ways to speak. better assist student achievement and language competence. $3 \quad 13 \%$ deprive speakers and listeners of language resources. $3 \quad 13 \%$

$4 \quad 17 \%$

$13 \quad 57 \%$

Number and Percentage of

8. Black English Dialect Participants

carries complex information across a range of cultural groups. $522 \%$ conveys much meaning for experienced speakers and listeners. $12 \quad 52 \%$ is accepted as a viable communication mode in society today.

$4 \quad 17 \%$

should be discouraged in public school settings.

$29 \%$

12. It is necessary to know about English-speaking cultures in order to speak and understand English.

Number and Percentage of

\begin{tabular}{llc}
\hline True & 18 & $78 \%$ \\
False & 5 & $22 \%$ \\
\hline
\end{tabular}

17. People who speak many languages

Number and Percentage of are intelligent. Participants should focus on one.

$18 \quad 78 \%$
get confused easily. $14 \%$

. can only truly be proficient in one of the languages.

$0 \quad 0 \%$

21. In the US, who is primary responsibility of ensuring appropriate $14 \%$ instruction of English language learners?

Number and Percentage of General classroom teachers

ESL teachers Participants

Language Arts teachers $14 \%$

all teachers are equally responsible

$29 \%$

$14 \%$

$18 \quad 78 \%$

Number and Percentage of

24. Monolingual/monocultural policies in schools, Participants create equality among students. $0 \quad 0 \%$ benefit students by teaching language and culture of power. $4 \quad 17 \%$ create student failure by exclusion of multiple perspectives.

$11 \quad 48 \%$ 
divide students further due to lack of acknowledgment of difference.

$8 \quad 35 \%$

26. Academic success will fall if teachers allow Black English to be spoken in school.

Number and Percentage of

Strongly agree Participants

Agree

$0 \quad 0 \%$

Neither agree or disagree

$14 \%$

Disagree

$6 \quad 26 \%$

Strongly disagree

$8 \quad 35 \%$

27. Speaking Black English limits a student's comprehension of other languages.

$5 \quad 22 \%$

\section{Strongly agree}

Agree

Neither agree or disagree

Disagree

Strongly disagree

Number and Percentage of Participants

30. The elimination of nonstandard dialects of English creates social stability.

$0 \quad 0 \%$

$0 \quad 0 \%$

$6 \quad 26 \%$

$8 \quad 35 \%$

$6 \quad 26 \%$

Number and Percentage of

Strongly agree Participants

Agree

Neither agree or disagree

$0 \quad 0 \%$

$14 \%$

Disagree

$3 \quad 13 \%$

Strongly disagree

$10 \quad 43 \%$

31. As a part of the

31. As a part of the Chinese culture, Mandarin dialects should be encouraged in the classroom.

$6 \quad 26 \%$

Number and Percentage of

Strongly agree Participants

Agree

$0 \quad 0 \%$

Neither agree or disagree

$939 \%$

Disagree

$1148 \%$

Strongly disagree

$0 \quad 0 \%$

33. Society does not benefit from usage of nonstandard dialects of

English.

$0 \quad 0 \%$

Number and Percentage of

Strongly agree Participants

Agree

Neither agree or disagree

$0 \quad 0 \%$

$0 \quad 0 \%$

Disagree

$6 \quad 26 \%$

$8 \quad 35 \%$

Strongly disagree

$6 \quad 26 \%$

35. A teacher should correct a student's use of nonstandard English.

Number and Percentage of

Strongly agree Participants

Agree

$0 \quad 0 \%$

$7 \quad 30 \%$

Neither agree or disagree

$10 \quad 43 \%$ 
Disagree

$4 \quad 17 \%$

Strongly disagree

$0 \%$

\section{Linguistic Knowledge Findings}

Research Question 2: What linguistic knowledge do preservice teacher candidates have at the beginning of a teacher preparation program?

Foundational linguistic knowledge in areas of phonology, syntax, semantics, pragmatics, culture, instructional methods, and brain processing of language were found to be important components that inform teacher language awareness from the literature. Linguistic knowledge allows teachers to provide a communication-friendly environment and focus on cultural and linguistic differences represented in the classroom without the necessary implication of deficits. Having basic linguistic knowledge allows teachers to provide support for and create opportunities for social and professional language use. Findings in this study are limited, but provide important information for further study.

Phonological knowledge findings. The participants in this study had varying levels of phonological knowledge (see Table 4.6). The articulation of sound was the weakest knowledge category. Only $17 \%$ of the participants knew the difference between place and manner of articulation and only one participant had any knowledge of aspiration and the relationship of breathing and speaking. Syllabification was another weak area. Only one participant scored 4 out of 4 on breaking words down into syllables. When counting the number of syllables $43 \%$ of the participants knew at least two out of four. During interviews, participants (48\%) reported knowledge about the importance of phonology when trying to understand speech, but further explained that they did not 
know all of the rules for applying phonological knowledge to their speech or their students'. Further, $65 \%$ of the participants had knowledge of how to parse words into sounds. Knowledge about rhyming words and ending words sounds was higher than other phonological knowledge. Seventy-four percent of participants were able to match the ending sounds in words. Though many of the participants had taken linguistics classes and gained phonological knowledge, Participant B shared a common sentiment among those that had not learned about phonology, "I don't know enough about the specifics of these questions. I know and use language, but do not recall learning about the physical aspects of language specifically."

From clearly hearing phonemes to the articulation of language production, teachers should have the ability to negotiate phonological discrepancies. Few participants exhibited enough knowledge in the physical aspect of articulation to evaluate a language perception or production issue. There is also indication that participants do not understand the difference between a phonological error versus a phonetic error which has been proven in the research to lead to misdiagnosis of student's abilities and misinterpretation of necessary instruction. As explained by Buckingham and Christman (2008), teachers often focus on the "correct" pronunciations required in proficient language use but disregard the importance of the how those sounds are produced. Understanding the physiology of proficient language production is often ignored in classrooms. Foundational knowledge in phonetics and phonology of language provide information for interpretation and negotiation of diverse discourse. This knowledge also provides teachers with language awareness for their own language choices. 
Table 4.6

\section{Phonological Knowledge}

\begin{tabular}{|c|c|c|}
\hline 7. The only difference between the phoneme $/ \mathrm{k} /$ and $/ \mathrm{d} /$ is & \multicolumn{2}{|c|}{$\begin{array}{l}\text { Number and Percentage of } \\
\text { Participants }\end{array}$} \\
\hline *Place of articulation - the location in the mouth of the speech sound & 4 & $17 \%$ \\
\hline $\begin{array}{l}\text { Manner of articulation - how the tongue, lips, and jaw are involved } \\
\text { in making a speech sound }\end{array}$ & 6 & $26 \%$ \\
\hline Aspiration - the act of breathing during a speech sound & 1 & $4 \%$ \\
\hline Both $a$ and $b$ & 12 & $52 \%$ \\
\hline $\begin{array}{l}\text { 9. Considering pronunciation, not orthography (writing), circle how } \\
\text { many syllables the following words have: }\end{array}$ & \multicolumn{2}{|c|}{$\begin{array}{c}\text { Number and Percentage of } \\
\text { Participants }\end{array}$} \\
\hline Lightening $2 * 3 \quad 4$ & 7 & $30 \%$ \\
\hline Spoil $122 * 3$ & 1 & $4 \%$ \\
\hline Walked $1 * 2 \quad 3$ & 5 & $22 \%$ \\
\hline Decidedly $3 * 4 \quad 5$ & 20 & $87 \%$ \\
\hline 10. How many phonemes (speech sound) in the word edge? & \multicolumn{2}{|c|}{$\begin{array}{l}\text { Number and Percentage of } \\
\text { Participants }\end{array}$} \\
\hline 1 & 0 & $0 \%$ \\
\hline$* 2$ & 16 & $70 \%$ \\
\hline 3 & 7 & $30 \%$ \\
\hline 4 & 0 & $0 \%$ \\
\hline
\end{tabular}

\begin{tabular}{lcc} 
11. What is the third speech sound in wretch? & Number and Percentage of \\
\hline$* / \mathrm{ch} /$ & 15 & $65 \%$ \\
/e/ & 6 & $26 \%$ \\
/t/ & 1 & $4 \%$ \\
\hline
\end{tabular}

12. Do hut and foot rhyme in your speech?

Number and Percentage of

\begin{tabular}{lcc}
\hline Always/ usually rhyme & 0 & $0 \%$ \\
Sometimes rhyme & 5 & $22 \%$ \\
Never/very rarely rhyme & 18 & $78 \%$ \\
\hline
\end{tabular}

13. For the following words, find a word in the row that ends with the same sound:

Number and Percentage of

\begin{tabular}{lcccc}
\hline Dogs: *his ducks piece & 10 & $43 \%$ \\
Knew: sew coy *igloo & 18 & $78 \%$ \\
Shrink: thing *antique fatigue & 18 & $78 \%$ \\
\hline
\end{tabular}

14. Where in the mouth is the sound /T/ - /th/ in think?

Number and Percentage of

*Front of the mouth (interdental) Participants

$2191 \%$ 


\begin{tabular}{lccc} 
Back of the mouth (velar) & 0 & $0 \%$ \\
Throat (glottal) & 1 & $4 \%$ \\
\hline & \multicolumn{2}{c}{$\begin{array}{c}\text { Number and Percentage of } \\
\text { Participants }\end{array}$} \\
\hline Do these words rhyme? & Yes $=$ & 8 & $35 \%$ \\
\hline Merry and Murray & Yes $=$ & 10 & $43 \%$ \\
Spider and Writer & Yes $=$ & 13 & $57 \%$ \\
Sock and Talk & Yes $=$ & 17 & $74 \%$ \\
Water and Hotter & &
\end{tabular}

*Indicates the correct answer

Grammar knowledge findings. Participants in this study had a range of knowledge of syntactical features in the English language (see Table 4.7). Many of the participants (54\%) stated growing up in an environment that supported "correct use of grammar," however, in the syntax section of the assessment, they indicated they were unfamiliar with the terms and topics. Over half of the participants $(57 \%)$ were able to identify morphemes when given the definition. In contrast, $83 \%$ of the participants understood implications of providing correct English sentence structure. These participants also understood that comprehensible sentences might lack formal structure. Participants were able to offer analysis of a student's understandings pertaining to grammatically correct sentences versus semantically correct sentences. Participant B also commented that students should only be corrected if the statement is grammatically incorrect. This participant further explained that use of other forms of English would render a statement incorrect and would therefore need correction. When asked about plurality and negation, only one participant knew about language structure in other dialects or languages although some participants (48\%) explained that all dialects and languages had rules for altering words to change the tense or plural status. Additionally, they expressed no knowledge of Chomsky's theory of universal grammar. 
Participants (57\%) in this study had some knowledge of morphology and why it was necessary to know. The majority (83\%) reported an understanding of syntax. They had knowledge about parts of speech. Further, they knew, for example, that by substituting a noun for a noun, the syntax of a sentence would remain the same, but the sentence would not make sense. This knowledge was predominantly understood in English. When asked about other languages, few expressed this type of knowledge. This knowledge will help these preservice teachers partially in their own English speech, but is limiting with regard to the relationship of English and other languages that might be spoken in the classroom.

Table 4.7

\begin{tabular}{|c|c|c|}
\hline $\begin{array}{l}\text { 5. An example of a morpheme, the smallest units of sound with } \\
\text { meaning, is }\end{array}$ & \multicolumn{2}{|c|}{$\begin{array}{l}\text { Number and Percentage of } \\
\text { Participants }\end{array}$} \\
\hline *The /s/ in cats. & 13 & $57 \%$ \\
\hline Cat, the whole word. & 6 & $26 \%$ \\
\hline Both $a$ and $b$. & 3 & $13 \%$ \\
\hline Neither a nor $b$. & 0 & $0 \%$ \\
\hline $\begin{array}{l}\text { 16. A teacher asks a student to fill in the blank to the sentence, } \\
\text { "Mary fell off the }\end{array}$ & \multicolumn{2}{|c|}{$\begin{array}{l}\text { Number and Percentage of } \\
\text { Participants }\end{array}$} \\
\hline The student is displaying meaning and grammar knowledge. & 1 & $4 \%$ \\
\hline The teacher should teach the student more vocabulary. & 0 & $0 \%$ \\
\hline *The student is displaying grammar, but not meaning knowledge. & 19 & $83 \%$ \\
\hline The student is not displaying any knowledge of language systems. & 2 & $9 \%$ \\
\hline
\end{tabular}

*indicates the correct answer

Semantic knowledge findings. Although participants expressed the importance of creating mutual comprehension in interactions with students, these participants lacked deep knowledge of specific aspects of language which scholars considered important (see Table 4.8). Participants (52\%) in this study reported that understanding the accent of the speaker was the hardest part of understanding continuous speech. During interviews, 
participants (54\%) in this study, Participant $\mathrm{P}$ expressed that "the more diversity, the better with regard to language interactions. But if the accent is too thick, students might be disadvantaged by their lack of understanding continuous speech." Many of these participants $(62 \%)$ explained their experiences abroad influenced their answers to this question. They expressed that from their personal experiences, they perceived the most difficult part of distinguishing meaning was because of accents. Few participants (13\%) in this study reported that the hardest part of comprehension is hearing word boundaries in speech.

Seventy-four percent of the participants reported that comprehension of language relies initially on word and sentence level meaning, and then concept recognition. However, when asked in interviews about the relationship of word and sentence structure and comprehension, only $17 \%$ of participants reported significance in the relationship. Further interview questions revealed a lack of understanding about deep and surface sentence structures. Wilde (2000) contended that teachers need to take into account the student's spoken language when instructing in a classroom. She explained that students that speak another form of English, especially one that is considered lower-status is often considered to be evaluated as incorrect because of surface structure differences. Moreover, Labov (1994) asserted, languages and dialects carry deep and surface structure. Cultural influences on language change these structures. Two sentences that are stated differently can mean the same thing. Although the research highlights judging a student's speech by asking whether the spoken sounds are similar to the student's everyday speech instead of are the spoken sounds similar to Standard English, only 22\% 
of the participants agreed that meaning can remain the same when sentence structure changes. It is important to understand how the teacher evaluates student speech because it influences how the teacher uses language in the classroom. Even though $65 \%$ of the participants added that different sentence structures in language denote cultural influences, $13 \%$ of the participants reported that sentence structure variation displays correct or incorrect versions of English. These results show that many of these participants are comparing students' native language to Standard English. Findings also indicate that participants demonstrate general semantic knowledge, but not suggested with examples of how a non-native language speaker would hear or speak English or how a teacher could use this information in their own speech.

Although participants in this study perceived their understandings of speech as predominantly accent issues, Yeni-Komshian (1998) explained that continuous speech is difficult to decode because language tends to be paced at about 125-180 words per minute. She added that the signal is complex and continuous and was found to be the critical component in distinguishing language. Acoustic boundary markers are difficult to hear for listeners new to the language. In the beginning of language understanding, phonemes are not automatically retrieved from memory banks in the brain. Speech perception research also explained that listeners have to ascertain boundaries of speech, as well as to define conceptual boundaries of language (Gleason \& Ratner, 1998). Semantic verification requires knowledge of certain features. Findings in this study indicate varied knowledge of components that are necessary for meaning making. Those participants who had longer and deeper experiences with learning second and third 
languages had a better understanding of comprehension in English. However, few participants had knowledge of sentence structure variation with relation to meaning.

Table 4.8

Semantic Knowledge

\begin{tabular}{|c|c|c|}
\hline 6. The hardest part of understanding continuous speech is & \multicolumn{2}{|c|}{$\begin{array}{c}\text { Number and Percentage of } \\
\text { Participants }\end{array}$} \\
\hline picking out the grammar. & 3 & $13 \%$ \\
\hline hearing word boundaries. & 3 & $13 \%$ \\
\hline understanding the accent. & 12 & $52 \%$ \\
\hline deciding what is meaningful. & 5 & $22 \%$ \\
\hline 15. Comprehension of language relies on & \multicolumn{2}{|c|}{$\begin{array}{c}\text { Number and Percentage of } \\
\text { Participants }\end{array}$} \\
\hline the use of standard language. & 0 & $0 \%$ \\
\hline word and sentence level meaning, and concept recognition. & 17 & $74 \%$ \\
\hline using the technique, "repeat after me." & 1 & $4 \%$ \\
\hline knowledge of the culture surrounding the language. & 4 & $17 \%$ \\
\hline $\begin{array}{l}\text { 19. I asked Alvin if he knew how to play basketball and I axt Alvin } \\
\text { does he know how to play basketball are examples }\end{array}$ & \multicolumn{2}{|c|}{$\begin{array}{l}\text { Number and Percentage of } \\
\text { Participants }\end{array}$} \\
\hline of correct and incorrect English dialects & 3 & $13 \%$ \\
\hline of different ways to say the same thing & 5 & $22 \%$ \\
\hline of the influence of inappropriate instruction & 15 & $65 \%$ \\
\hline of bilingualism & 0 & $0 \%$ \\
\hline
\end{tabular}

Pragmatic knowledge findings. Language change occurs in every language and affects generations of comprehension. Sixty-one percent of participants reported that language variation most often occurs at the semantic level. Twenty-nine percent of the participants reported that language most frequently changed at the phonetic level. In interviews, a few of these participants (23\%) stated that language change was important for teachers to understand because a teacher's interpretation of correct language use might be affected by changes in language. Participant V explained that "keeping up with 
the progression of language might mean not speaking Standard language. Standard might be outdated." Mahony (2011) explained that the most frequently studied type of language change is at the vocabulary/semantic level of language because of the relatively rapid change in the language at that level. Phonetic changes are harder to study and the resulting change is not always definitively language change, but sometimes accent variation. Participants in this study had an understanding of language change, but most (77\%) felt it was unimportant knowledge. However, Randall (1999) explained that an understanding of language change is important because language forms our attitudes. He explained that language is how we communicate and how we think. Rigid, unchanging views of language often reveal underlying attitudes of strictly interpreted usage. To use language effectively, especially with diverse populations, an understanding of language as a dynamic tool that shapes our world view is important. When language changes; we change. The awareness helps teachers negotiate language issues with students.

\section{Table 4.9}

\section{Pragmatic Knowledge}

\begin{tabular}{lcc}
\hline & \multicolumn{2}{c}{ Number and Percentage of } \\
8. Language change most often occurs at: & \multicolumn{2}{c}{ Participants } \\
\hline a phonetic level - individual speech sounds & 6 & $26 \%$ \\
a morphological level - word structure & 2 & $9 \%$ \\
a syntactic level - grammatical & 0 & $0 \%$ \\
a semantic level - literal meaning & 14 & $61 \%$ \\
\hline \multirow{2}{*}{ 23. Language use and comprehension are most dependent on which } & Number and Percentage of \\
three aspects: & \multicolumn{2}{c}{ Participants } \\
\hline Vocabulary, context, rhyming & 3 & $13 \%$ \\
Speaker, listener, context & 6 & $26 \%$ \\
Expression, grammar, volume & 0 & $0 \%$ \\
Visuals, context, references & 14 & $61 \%$ \\
\hline
\end{tabular}


Cultural knowledge findings. Despite the fact that $22 \%$ of the participants did not know the meaning of the term code-switching, many participants explained during interviews that the concept of switching languages was beneficial. After the term was explained, $52 \%$ of the participants provided good understanding of reasons for switching language. They reported that clarifying and elaborating information, marking identity, and showing power were all valid reasons for switching language. However, in practical terms, the participants expressed a lack of knowledge about the use of code-switching in the classroom, especially "if initiated by the student." Participant C argued that "all students must learn the importance of the concept of code-switching."

Additionally, $87 \%$ of the participants had knowledge of the restrictions that come with only speaking one language or dialect. Forty-eight percent of the participants reported that speaking one dialect means that students may not hear the sounds from another dialect. Furthermore, 39\% of the participants reported that speaking one dialect limits understanding of other dialects.

Although all of the participants had knowledge of the influence culture had on language use, the particular aspects of culture that would influence the classroom were thought of differently. In relation to the theories mentioned in Chapter 2 of this study, participants revealed varying degrees of understanding of the importance of codeswitching, the limitations of speaking only one dialect, and the influence of cultural variation on language structure. Participants in this study also indicated little knowledge about cultural influences on languages other than Standard English. The lack of experience with cultures and the lack of understanding of how culture influences 
language use have been described in the literature (Halliday, 1975; Moll, Saez, \&

Dworin, 2001; Scribner \& Cole, 1981). Teachers must understand how culture influences language experiences with diverse populations.

Table 4.10

Cultural Knowledge

\begin{tabular}{lcc}
\hline 3. Teachers and students switch languages to & Number and Percentage of \\
\hline clarify and elaborate information. & 8 & $35 \%$ \\
mark their identity. & 1 & $4 \%$ \\
show power. & 2 & $9 \%$ \\
All of the above. & 12 & $52 \%$ \\
\hline & Number and Percentage of \\
18. Speaking one dialect & Participants \\
\hline limits understanding of other dialects & 9 & $39 \%$ \\
allows students to speak to more people & 0 & $0 \%$ \\
*means that students may not hear sounds from another dialect & 11 & $48 \%$ \\
enhances student performance in language activities & 3 & $13 \%$ \\
\hline 19. I asked Alvin if he knew how to play basketball and I axt Alvin & Number and Percentage of \\
does he know how to play basketball are examples & Participants \\
\hline of correct and incorrect English dialects & 3 & $13 \%$ \\
*of different ways to say the same thing & 5 & $22 \%$ \\
of the influence of inappropriate instruction & 15 & $65 \%$ \\
of bilingualism & 0 & $0 \%$ \\
\hline & \multicolumn{2}{c}{0} \\
23. Black English lacks basic concepts like plurality and negation. & Number and Percentage of \\
\hline *True & Participants \\
False & 2 & $9 \%$ \\
\hline
\end{tabular}

Instructional knowledge findings. To instruct diverse student populations, teachers need an understanding of what parts of language play a role in affecting classroom instruction (see Table 4.11). Seventy-eight percent of the participants reported that cognitive elements such as sounds, meanings, and grammar of language are most important in the development of language skills. During interviews, $46 \%$ of the participants agreed with Participant G when she said, "during instruction it is important to 
think about what you say." Participant F added that "making sure you are speaking correct English and meaning what you say is an obvious part of instruction." Fifty-two percent of the participants also understood that code-switching would play an important role in classroom language use. The majority of participants $(78 \%)$ had knowledge of effective intercultural communication, stating that this would require sensitivities to the social aspects of language and knowledge of culture and language relationships. All participants stated that it would require the social or cultural knowledge for effective intercultural communication.

Participants in this study were divided on the type of instructional environment that would be best for diverse populations. Though 39\% reported in the assessment that explicit instructions and a quiet environment were best practice, only $26 \%$ reported in interviews that a structured environment that allowed for frequent discussion was better suited to diverse populations. Fillmore, Ammon, McLaughlin, and Ammon (1985) compared bilingual and English only classrooms and instructional strategies. They found that different environments worked differently depending on the background of the students. For example, Chinese students exhibited gains in language perception in classrooms where instruction was structured and explicit. On the other hand, Latino students exhibited gains in comprehension with structured instruction followed by practice in a low noise level environment. Chinese students also benefited from peer interaction, but only after they reach intermediate English proficiency whereas Latino students benefited from peer interaction from the beginning of English learning. Although studies have found that interactions matter, classrooms are still described in the 
literature as passive language environments that limit student's opportunities to interact to develop language proficiencies. The findings in this study were consistent with the literature.

Table 4.11

Instructional Knowledge

1. To develop language skills, students must have knowledge of the Number and Percentage of cognitive elements such as Participants

\begin{tabular}{lcc}
\hline *the sounds, meanings, and grammar of language. & 18 & $78 \%$ \\
culture and sounds of the language. & 3 & $13 \%$ \\
stresses on words and sentences. & 0 & $0 \%$ \\
knowing the correct terminology for talking about language. & 0 & $0 \%$ \\
\hline & Number and Percentage of \\
4. Effective intercultural communication & Participants \\
\hline Requires sensitivities to the social interactional aspects of language. & 3 & $13 \%$ \\
Uses knowledge of culture and language & 1 & $4 \%$ \\
$*$ Both a and b. & 18 & $78 \%$ \\
Neither a and b. & 0 & $0 \%$ \\
\hline & \multirow{2}{*}{ Nhe best type of instructional environment for diverse populations is } & Participants \\
\hline explicit instruction and quiet environment & 9 & $39 \%$ \\
flexible instruction and environment & 5 & $22 \%$ \\
structured and quiet & 39 \\
$*$ structured with frequent student discussion & 3 & $13 \%$ \\
\hline
\end{tabular}

Brain processing knowledge findings. Findings in brain processing of language indicated uncertainty from all of the participants (see Table 4.12). Fifty-seven percent of the participants stated that the inability to identify sounds in words predominantly created deficits in the ability to process and produce language. However, $35 \%$ of the participants stated that the lack of word pronunciation and memory skills created deficits in the ability to process and produce language. 
The participants (35\%) reported that the key to learning language and learning through language is to create more synaptic connections by experiencing the language. In addition, $30 \%$ of the participants added that memory skills were important to developing language processing abilities. Eighty-three percent of the participants agreed that students remembered language better when teachers used both implicit and explicit methods of instruction. When asked to explain or list specific strategies about embedding language into memory, the only method revealed was the use of repetition.

The importance of understanding the process of memory retrieval and storage is critical when considering the design of instruction. During interviews, all participants revealed knowledge of the importance of stimulating brain function, but no knowledge of the specifics of memory formation in relation to language use. When asked about the best strategies to embed information into long term memory, findings in this study revealed little knowledge of the crucial role that emotions, relevancy, novelty, attention, interest, and social components play in language learning and use. By connecting brain research to student learning, teachers can provide effective methods of instruction (Sousa, 2010). Teachers who are educated about cerebral processing and language reception as well as production are better equipped to address cultural and linguistic diversity with their language of instruction.

Table 4.12

Brain Processing Knowledge

2. Deficits in the ability to process and produce language are due predominantly to

*lack of word pronunciation and memory skills. lack of writing skills.

Number and Percentage of Participants

$8 \quad 35 \%$

$14 \%$ 
lack of identifying sounds in words.

lack of spelling-sound rules.

$13 \quad 57 \%$

$0 \quad 0 \%$

Number and Percentage

17. The key to learning language is to create synaptic connections by of Participants

*experiencing language

$8 \quad 35 \%$

Repetition

$3 \quad 13 \%$

getting the 'right' answer

$6 \quad 26 \%$

stimulating the left hemisphere

$4 \quad 17 \%$

28. Students remember more information when they use a process that involves implicit and explicit learning.

\begin{tabular}{lcc} 
*Strongly Agree & 6 & $26 \%$ \\
*Agree & 13 & $57 \%$ \\
Neither agree or disagree & 1 & $4 \%$ \\
Disagree & 0 & $0 \%$ \\
Strongly disagree & 0 & $0 \%$ \\
\hline
\end{tabular}

\section{Monolingual and Multilingual Comparison Findings}

Research Question 3: What are the similarities and differences in the beliefs and knowledge about language between monolingual and multilingual preservice teachers?

Regardless of grouping methods, individuals never reflect a homogenous group ideal. Comparisons between the monolingual and multilingual participant groups in this study showed no significant differences in beliefs of language overall. However, there were findings worthy of mention.

All of the participants (100\%) in this study reported that a teacher's primary instructional focus should be ensuring comprehension of all students. This belief was the only unanimous agreement between all of the participants. The majority of both monolingual and multilingual participant also agreed that comprehending a language requires knowing the vocabulary and the syntax. Monolingual (82\%) and multilingual (83\%) participants also had similar views about who within a school is responsible for ensuring appropriate instruction of diverse populations. They reported that all teachers; 
general education, ESL, and language arts teachers as well as administrators should play a role in supporting students that need support. Most participants (83\%) also agreed that monolingual and monocultural policies created failure, exclusion, and divided students by their differences.

During interviews, both monolingual and multilingual participants also agreed that the context in which language is used is very important. Overall, most participants (70\%) felt teaching students to understand the contexts in which to use different languages and registers was beneficial for students. Although the research literature highlights the fact that multilingual teachers have an advantage over monolingual teachers (Bialystok, 2007; Valencia, 2011), the agreement between groups in this study indicate a possibility that monolingual teachers might share important beliefs with multilingual teachers. These findings demonstrate that the disparity between teacher and student background might not be as significant as the literature portrays if all teachers are supported.

Despite the encouraging similarities between these groups that appear to reveal these preservice teachers awareness of certain key items of diversity such as the use of student's linguistic background, there were areas in which monolingual and multilingual participants differed. During interviews, most participants (77\%) reported that they believed that diversity is a resource. Participant M stated, "recognizing the diverse backgrounds of my students and using that diversity as a pedagogical tool will help me create learning environments of respect. Diversity in learning strengths will push me to incorporate multiple modalities." Moreover, Participant S explained that "languages of 
others [students] in the classroom can help build our classroom community as a positive place." However, significantly echoing the beliefs laid out in the literature, these monolingual participants could not explain exactly how they would integrate all students' backgrounds and teach all of the content in the classroom where as similarly situated, multilingual participants reported the ability to use the languages present in the classroom in specific ways to celebrate everyone. This is important because it reveals the significance for teacher preparation programs to design courses that explicitly teaching preservice teachers how to incorporate student diversity in the classroom. Multilingual participants also explained that drawing on their experiences with various languages would help them with negotiating language issues and creating broader curricula. As Participant Y explained it is "because I understand the differences in grammars/logics and more between two languages, I will be able to put myself in a language learner's shoes, think for him or her, and design a class that is guided not just by mainstream theories that are based on mainstream standards."

Monolingual and multilingual participants also reported differences in the area of teacher's language use in the classroom. In this study, monolingual participants (59\%) reported that teachers have a responsibility to teach only Standard English to insure that students use the most widely understood language. Conversely, multilingual participants $(83 \%)$ reported that it is a teacher's responsibility to teach many dialects and registers of English to help students negotiate all language. Many scholars agree that to create equitable learning environments in schools, teachers must explicitly teach about various language use (Baker, 2002; Baker, Kovelman, Bialystok, \& Petitto, 2003). However, 
Wong, Berta-Avila, William-White, Baker, Arellano, and Echandia (2007) explained that though moving teachers toward this utopian vision is important, it creates significant challenges for teacher preparation programs. It is possible that the lack of experiences and knowledge that monolingual participants have with variants of language could cause the discrepancy. The evidence in this study shows that the multilingual participants have a greater understanding and more empathy toward language learning because of their varied experiences with different language contact. Teacher language awareness was greater in the multilingual participants.

The amount of time believed needed to become proficient in a language differed between the two groups. The majority of monolingual participants (69\%) reported 1-5 years would be an appropriate amount of time. The majority of multilingual participants (67\%) reported 6-9 years would be required to become proficient. It appeared that knowing the quantity of time required to learn and understand a $2^{\text {nd }}$ language was better understood by the multilingual participants. Monolingual Participant A explained during the interview that he thought that "if a school system required a foreign language for only two years then they should know what it takes to create language users. I did not learn in that period of time, but I did not care about becoming proficient, it was a requirement I had to complete to graduate." This comment indicates that it is possible there is a lack of motivation to learn another language and might blame themselves for not learning or it might not occur to them that the timeframe of 2 years is too short to become proficient. In contrast, all multilingual participants reported that their experiences with learning a language reflect a much longer and more realistic time period. The personal experiences 
seem to have influenced their understanding of language use as a time-consuming endeavor. Those participants who had more experiences with diversity had more realistic views about the length of time it takes to understand a language. The monolingual participants rationalized that they had not learned another language in the two year requirement for high school. Instead of believing that the timeframe was too short to learn, the monolingual participants expressed a trust in the education system that two years was enough. Participant G explained that she "thought that the schools would have researched the appropriate time for acquisition" and that she had not really thought about why she was not proficient in another language. She accepted a systems explanation that undermined her own self-knowledge and might impact her ability to relate to ELL students in her classroom.

Additionally, the relationship between culture and language was more deeply understood by the multilingual participants. Of the multilingual participants, $100 \%$ reported that it is necessary to learning about the culture to have a full understanding of the language. In contrast, $58 \%$ of monolingual participants reported that culture is necessary to speak and understand a language. This comparison indicates that monolingual participants might hold the belief that language understandings come from the ability to learn the vocabulary and grammar of a language to communicate. In other sections of this study, participants have stated that comprehension is possible without a full understanding of the grammar rules. These results beg the question: can deep comprehension occur without an understanding of the rules of the language and the culture? It is possible that further exploration into this aspect of language is necessary. 
Monolingual participants and multilingual participants had a strong difference of opinion regarding how a teacher should speak in a classroom. Monolingual participants stated that it is important for a teacher to either use academic English with perfect pronunciation or not teach students directly. By contrast, multilingual participants stated that it is important for teachers to use the local dialect of English or speak English with varying pronunciation to all students' experiences with other forms of English. These results explain why it is crucial that teacher preparation programs address these issues with preservice teachers.

When asked about the difficulty of the English language, monolingual and multilingual participants expressed different perspectives. Monolingual participants $(52 \%)$ reported that English is a very difficult language to understand and speak. Yet, $67 \%$ of multilingual participants stated that the difficulty of understanding and speaking English is different for everyone. Responses from participants suggest that language awareness at different levels influences the perceptions about the difficulty language.

Table 4.13

Belief comparisons of monolingual and multilingual participants

\begin{tabular}{|c|c|c|c|c|}
\hline Beliefs & \multicolumn{2}{|c|}{$\begin{array}{l}\text { Monolingual } \\
\text { Participants }\end{array}$} & \multicolumn{2}{|c|}{$\begin{array}{l}\text { Multilingual } \\
\text { Participants }\end{array}$} \\
\hline A teacher's primary instructional focus should be & \multirow[b]{2}{*}{17} & \multirow[b]{2}{*}{$100 \%$} & \multirow[b]{2}{*}{6} & \multirow[b]{2}{*}{$100 \%$} \\
\hline ensuring the comprehension of all students. & & & & \\
\hline The most important aspect of comprehending a speaker is to know & \multirow[b]{2}{*}{11} & \multirow[b]{2}{*}{$65 \%$} & \multirow[b]{2}{*}{5} & \multirow[b]{2}{*}{$83 \%$} \\
\hline not just one of the aspects including vocabulary, syntax, accent. & & & & \\
\hline $\begin{array}{l}\text { In the U.S., who has primary responsibility of ensuring appropriate } \\
\text { instruction of English language learners? }\end{array}$ & \multirow[b]{2}{*}{14} & \multirow[b]{2}{*}{$82 \%$} & \multirow[b]{2}{*}{5} & \multirow[b]{2}{*}{$83 \%$} \\
\hline all teachers are equally responsible. & & & & \\
\hline
\end{tabular}




\begin{tabular}{|c|c|c|c|c|}
\hline \multicolumn{5}{|l|}{ Monolingual/monocultural policies in schools } \\
\hline create failure by exclusion of multiple perspectives. & 7 & $41 \%$ & 4 & $67 \%$ \\
\hline $\begin{array}{l}\text { divide students further due to the lack of acknowledgment of } \\
\text { differences. }\end{array}$ & 7 & $41 \%$ & 2 & $33 \%$ \\
\hline \multicolumn{5}{|l|}{ In the U.S., it is a teacher's responsibility to } \\
\hline $\begin{array}{l}\text { teach Standard English only in order to insure that students use the } \\
\text { most widely understood language. }\end{array}$ & 10 & $59 \%$ & 0 & $0 \%$ \\
\hline $\begin{array}{l}\text { teach many dialects and registers of English in order to help students } \\
\text { negotiate all language use. }\end{array}$ & 1 & $0.06 \%$ & 5 & $83 \%$ \\
\hline teach World Englishes that exist in the classroom. & 3 & $17 \%$ & 1 & $17 \%$ \\
\hline \multicolumn{5}{|l|}{$\begin{array}{l}\text { If someone spent one hour a day learning language, how long would it } \\
\text { take to become proficient? }\end{array}$} \\
\hline $1-2$ years & 2 & $12 \%$ & 0 & $0 \%$ \\
\hline $3-5$ years & 8 & $47 \%$ & 0 & $0 \%$ \\
\hline $6-9$ years & 3 & $17 \%$ & 4 & $67 \%$ \\
\hline You can't learn a language proficiently in one hour a day. & 4 & $12 \%$ & 2 & $33 \%$ \\
\hline \multicolumn{5}{|l|}{$\begin{array}{l}\text { It is necessary to know about English-speaking cultures to speak and } \\
\text { understand English. }\end{array}$} \\
\hline True & 12 & $71 \%$ & 6 & $100 \%$ \\
\hline False & 5 & $29 \%$ & 0 & $0 \%$ \\
\hline \multicolumn{5}{|l|}{ As a teacher, it is important to speak English } \\
\hline using academic English with perfect pronunciation. & 8 & $47 \%$ & 0 & $0 \%$ \\
\hline using the local dialect of English. & 2 & $12 \%$ & 5 & $83 \%$ \\
\hline correctly or not teach students directly. & 5 & $29 \%$ & 0 & $0 \%$ \\
\hline $\begin{array}{l}\text { with varying pronunciations to allow students experiences in World } \\
\text { Englishes. }\end{array}$ & 2 & $12 \%$ & 1 & $17 \%$ \\
\hline \multicolumn{5}{|l|}{ English is a } \\
\hline very difficult language to understand and speak. & 10 & $52 \%$ & 2 & $33 \%$ \\
\hline language of medium difficulty to understand and speak. & 3 & $17 \%$ & 0 & $0 \%$ \\
\hline different for everyone. & 3 & $17 \%$ & 4 & $67 \%$ \\
\hline
\end{tabular}

With regard to monolingual and multilingual participant groups, the findings indicate no significant difference in linguistic knowledge between groups. Table 4.14 reveals the most significant discrepancies in knowledge. Besides phonological knowledge, however, participants in both groups were diverse in their responses in other categories. One multilingual was able to answer all of the linguistic knowledge questions 
correctly and no monolinguals displayed this overall knowledge. Findings in this study about monolingual and multilingual linguistic knowledge are contrary to the literature discussed in Chapter 2. Furthermore, demographic findings revealed that monolingual participants had acquired linguistic knowledge through language learning and that most of them did not take specific courses in linguistics. All of the multilingual participants stated that they had taken courses in linguistics to understand language more precisely. Only a few of the monolingual participants took linguistics courses and stated that if "the courses had not been required, they probably would not have taken them" (Participant N).

The first overall finding was contradictory to the literature. Much of the literature described in Chapter 2 reported that multilingual teachers had advantages over monolingual teachers because of their metalinguistic knowledge (Ehri \& Nunes, 2002). Although other factors were important, teachers with accurate linguistic knowledge in categories such as phonology were better able to assess student's linguistic needs as well as manipulate their own language aiding in their students language awareness (Andrews, 1999). Many teachers in these studies were often multilingual. The literature describes multilingual teachers as having a better ability to facilitate explanation of phoneme categorization leading to specific language use than monolingual teachers. For example, if a student's first language is Japanese, the student may select an "l" where they should select an "r." Multilingual teachers are said to have a better grasp of this concept. Although this study found that multilingual participants did not exhibit more knowledge than monolingual, a conclusion cannot be drawn about their abilities to negotiate actual language issues. 
Both monolingual and multilingual participants had little knowledge of some aspects of phonology including syllabification. In contrast, both monolinguals and multilinguals were knowledgeable about other aspects of phonology including parsing and categorizing individual speech sounds. Two areas of phonology that were of interest were articulation and phonemic awareness. Interestingly, monolingual and multilingual participants alike answered half of the questions about articulation right and half of the questions wrong. One question asked specifically about the articulation of one sound and the other question asked participants to compare two sounds. Comparing sounds was problematic. Oral language requires knowledge of phonology and morphology, especially with multilingual students (McBride-Chang, Shu, Ng, Meng, Penney, 2007). McBride-Chang, Shu, Ng, Meng, and Penney (2007) contended that the phoneme /s/ denotes a sound that makes up a word, but it can also denote a plural. However, a plural can also use the speech sound /z/. For speakers of native languages other than English, confusion occurs when attempting to understand the phoneme in the word dress and the morpheme in the word cats or the speech sound at the end of buzz and the morpheme at the end of eggs. The argument of the importance of phonological and morphological knowledge can be made for all oral languages, particularly languages such as English which requires more inflected morphological knowledge than a language such as Chinese. Spoken language development increases for L2 speakers that have an understanding of phonological and morphological structures (McBride-Chang, Shu, Ng, Meng, Penney, 2007). 
Another area that proved to be problematic for some participants was the concept of rhyming. It is understandable that multilingual people might not rhyme in the same way as monolingual people. However, the results of the survey were convoluted enough to require further questioning during interviews to explore possible causes such as accent or language structure. During interviews, multilingual participants all revealed their lack of knowledge about the concept of rhyming and its relationship with phonemic awareness. Two multilingual participants defined the concept of rhyming incorrectly. Participant S explained that she thought that rhyming was more of a strategy for teaching phonemic awareness for learning English. She stated that though she remembered singing nursery rhymes in school in Mexico, she did not feel that it would have been important to focus on rhyming in Spanish. "It is more necessary in English because of all of the exceptions. As a teacher of English language learners, I use the strategy often to teach phonemic awareness and spelling rules, etc." While rhyming is only one strategy for developing phonological awareness, it is a well used strategy with English users as mentioned in the literature for assessing student language proficiency as well as determining the effectiveness of language use and reading ability. Additionally, McBride-Chang, Shu, Ng, Meng, Penney (2007), stated that English phonological awareness is more complex than in languages such as Chinese or German. For instance, consonant clusters in words such as split do not occur in other languages. August and Hakuta (1997) affirmed that a sophisticated knowledge of phonemes, as demonstrated by the ability to rhyme, segment, or group sounds, are key prerequisites to successful acquisition of language and reading. They further stated that teacher's knowledge in phonemic awareness promotes effective use of their own language as well as choice of 
instructional methods with diverse populations. However, they cautioned that findings to support these claims were based on monolingual English speakers. Because rhyming knowledge is linked to the ability to use language as well as understand specific aspects of language, teachers without the knowledge of rhyming might not be able to negotiate language use issues as effectively as needed. Multilingual participant responses might imply that they have more strategies available to them for negotiating language with the exception of rhyming strategies. As one strategy might not be sufficient when using language with diverse speakers, future studies exploring specific phonological awareness skills might be useful.

Table 4.14

Linguistic Knowledge Comparisons of Monolingual and Multilingual Participants

\begin{tabular}{|c|c|c|c|}
\hline Linguistic Knowledge & $\begin{array}{l}\text { Mo } \\
\text { PaI }\end{array}$ & $\begin{array}{l}\text { olingua } \\
\text { l } \\
\text { cipants }\end{array}$ & $\begin{array}{c}\text { Multilingua } \\
1 \\
\text { Participants } \\
\end{array}$ \\
\hline Do these words rhyme? & \multirow[b]{2}{*}{3} & \multirow[b]{2}{*}{$18 \%$} & \multirow[b]{2}{*}{$83 \%$} \\
\hline Merry and Murray & & & \\
\hline Spider and Writer & 6 & $35 \%$ & $67 \%$ \\
\hline Sock and Talk & 12 & $71 \%$ & $17 \%$ \\
\hline Water and Hotter & 11 & $65 \%$ & $100 \%$ \\
\hline How many phonemes (speech sounds) in the word egde? & \multicolumn{2}{|c|}{$\begin{array}{l}\text { Monolingual } \\
\text { Participants }\end{array}$} & $\begin{array}{l}\text { Multilingual } \\
\text { Participants }\end{array}$ \\
\hline 1 & 0 & $0 \%$ & $0 \%$ \\
\hline 2 & 10 & $66 \%$ & $66 \%$ \\
\hline 3 & 5 & $29 \%$ & $33 \%$ \\
\hline The 3 ingredients the brain needs for optimal language learning are: & \multicolumn{2}{|c|}{$\begin{array}{l}\text { Monolingual } \\
\text { Participants }\end{array}$} & $\begin{array}{l}\text { Multilingual } \\
\text { Participants }\end{array}$ \\
\hline relevance, emotion, context & 6 & $40 \%$ & $17 \%$ \\
\hline visual, kinesthetic, oral & 1 & $6 \%$ & $50 \%$ \\
\hline $\begin{array}{l}\text { I asked if he knew how to play basketball and I axt Alvin does he know } \\
\text { how to play basketball are examples of }\end{array}$ & \multicolumn{2}{|c|}{$\begin{array}{l}\text { Monolingual } \\
\text { Participants }\end{array}$} & $\begin{array}{l}\text { Multilingual } \\
\text { Participants }\end{array}$ \\
\hline of correct and incorrect dialects & 3 & $20 \%$ & $0 \%$ \\
\hline
\end{tabular}




\begin{tabular}{l|cc|cc}
\begin{tabular}{l|l} 
of different ways to say the same thing \\
of the influence of inappropriate instruction \\
of bilingualism
\end{tabular} & $\begin{array}{l}3 \\
9\end{array}$ & $60 \%$ & 5 & $83 \%$ \\
& 0 & $0 \%$ & 0 & $0 \%$ \\
\hline Black English lacks basic linguistic concepts like plurality and negation. & Monolingual & Multilingual \\
True & 2 & $12 \%$ & 0 & $0 \%$ \\
False & 6 & $35 \%$ & 6 & $100 \%$ \\
\hline
\end{tabular}

\section{Summary of Findings.}

Analysis of participants' language beliefs and linguistic knowledge was guided by the eight categories of knowledge derived from the literature (see Table 2.1) including the language acquisition process, phonology, syntax, semantics, pragmatics, culture, instruction, and memory. Emerging themes such as the influence of demographic information, influence of experience with other languages, and the context of language use guided the interpretation of the data. Answers to survey questions and interviews gave evidence of the beliefs and range of knowledge that participants have and provide information for strengthening teacher education programs.

Some participants in this study affirmed the importance of having empathy for what diverse student population's experience. They explained that this empathy aids in their effort to negotiate misunderstandings. Although some educators feel too constrained by the conditions of the educational system to always express their empathy (Cooper, 2004), empathy is a component of intercultural communicative competence which Deardorff (2006) argues is vital to seeking alternative ways to negotiate miscommunication and build trust with students. Delpit (1995) proposed that by using empathy (among other strategies), classroom teachers lead the way to offering diverse 
groups the opportunity to learn about each other without the presumption of privilege or domination by any member of the class. She explained that in order to achieve crosscultural dialogue that truly reveals what it might feel like to be someone else, "one must give up [their] own sense of who [they] are, and being willing to see [themselves] in the unflattering light of another's angry gaze" (p.46-47).

Although participants believed that understanding of many languages and dialects, understanding the relationship between language and culture, understanding language use in context, and understanding the benefits of being a multilingual individual was important in society, many participants felt these attributes did not play a significant role in the classroom. Even those who expressed that the value of these attributes aided in language understanding in the classroom also stated that incorporating them would be complex and felt they had "no idea of what this might look like" or felt that they "had little knowledge of how to do this," especially if there was more than a few different native languages spoken by the students.

Participants admitted to being uninformed about the terminology and the theories related to the process of language acquisition, but explained that their culture and language experiences gave them practical skills. Some of the participants (31\%) also revealed beliefs that their experience with exposure to languages motivated them to learn other languages. Unfortunately, these participants felt it was "normal" to be motivated by hearing other languages and that students that had exposure to other languages would learn languages faster and more effectively because of this motivation. This assumption revealed a bias that has implications for their own language use and student expectations 
as well as implications for teacher preparation programs. Overall, participants revealed beliefs that varied from the literature, but still expressed knowledge about the process of becoming proficient in language use. For example, participants in this study reported a belief in the time consuming nature of language acquisition, however, many participants believed that people could learn to use and comprehend a language in about three years according to the surveys. Researchers report that it takes closer to seven years to gain proficiency. Recognition of participants' personal experiences with learning another language seemed to absent when revealing expectations for student learning. For example, Participant A explained that he did not care about becoming proficient so therefore he didn't, but his students should become proficient in about three years. His expectation for students to become proficient might not take into consideration other factors such as motivation or learning style and negative evaluations of students' language use could result.

Findings in this study also diverged from the literature in beliefs about individual characteristics playing a larger role in acquisition than culture and environment influencing acquisition more powerfully than innateness. Additionally, as reflected in the literature, participants had differing views of competency. Although some participants argued that language competency was achieved by an understanding of the linguistic rules of a language, more participants stated that language competency was defined by reception of the underlying message with no reliance on the grammatical structure. Teacher preparation programs might explore balancing these two views. For example, Ovando (1997) contended that teachers should "strive to enable the English-language 
learner to develop phonology, morphology, syntax, and vocabulary primarily through real communicative activities rather than through such approaches as lecture and drills" (p. 288).

The systems in which a language is embedded were not understood in detail by these preservice teachers. However, their responses indicated an understanding of the complexities of language systems and the importance of using language appropriately as a tool to gain further understandings. Findings indicate the importance of gaining foundational awareness for different ways in which language systems work. The importance of understanding the concept that specific language systems are different is critical for developing teacher language awareness and a system of valuing languages as a resource for acceptance and respect of students.

While the participants advocated for Standard English use in the classroom, some expressed an understanding of the importance of maintaining their students' local and native dialects and languages. However, findings revealed a lack of skill in how to negotiate this complex dynamic issue in the classroom. Moll, Saez, and Dworin (2001) and Gonzalez (1984) emphasized that using native languages in the classroom does not sacrifice the acquisition of Standard English. Additionally, neurolinguists also emphasize the benefits of native languages during the mapping of new languages. Studies continue to show positive relationships between native language use in combination with English language learning and academic achievement (Ramos, 2009). Several questions on the survey addressed the use of other languages or dialects in the classroom. For instance, Standard English, Mandarin, Spanish, and Black English were each used as separate 
examples to establish beliefs in acceptable language use. Responses to these four questions were different even though the questions were worded the same. Eighty-two percent of the participants responded that Spanish should be used in the classroom, 57\% reported that Black English should be allowed in school, and 39\% of the participants stated Mandarin should be used by teachers and students for learning. More participants were unsure of the necessity to use Mandarin (48\%) than Black English (26\%) or Spanish (4\%). Participants were not available for inquiring about the reasons for the discrepancies after the data were analyzed. However, there could be many reasons for the discrepancies between responses such as familiarity of the language, comfort level in speaking and hearing the language, or the influence of societal marginalization of the language. Also, answers in other parts of the United States might be different. Participant A commented that using one classroom language creates easier access to mutual intelligibility because everyone is using one shared knowledge of one language. There was only one participant that was familiar with Mandarin, for instance, and he clarified when he believed it was appropriate to use Mandarin if at all. Forty-eight percent of the participants in this study explained that Standard English should be the only language spoken in the classroom.

Additionally, a few participants made comments concerning the large number of different native languages spoken in one classroom. Participant B stated, "I cannot possibly learn all of the languages so teaching in Standard English seems the most feasible." Though not obviously shared by other participants, she suggested a belief system that students needed to learn school language; she did not need to learn all of their 
languages. This perspective about language acquisition was commonly held for decades in the United States (Ravitch, 2001). The preservice teacher's perceptions of how society views the use of each language might also influence her view; "the consequences of notions of language supremacy" (Wynne, 2002, p. 208). Wynne explained that teacher beliefs, consciously or unconsciously, perpetuate language superiority or language inferiority by how they recognize or discount language representation in the classroom. Societal marginalization of Black English, for example, has long been reported in the literature. Some responses in this study implied belief in this marginalization. Seminal work in the literature from linguists and educators such as Heath (1983), Labov (1972), and others has changed the view of diverse language use from one of the deficit theory to one of equal but different. However, the perpetuation of a negative stigma with certain languages designates these languages as inferior to the dominant language and American, middle-class ways of using language.

Some participants $(13 \%)$ in this study reported that Black English is an incorrect version of Standard English. Studies by Labov (1972) provided evidence that AAVE uses systematic grammar and complex pragmatics making it as legitimate as other languages. Additionally, linguists have begun to substantiate the validity of other dialects and languages, especially within the communities that use them. Many participants in this study asserted that it is the teacher's responsibility to teach only in Standard English to ensure that students use the most widely understood language in the United States. This response combined with the responses about native language use (i.e. Spanish, Black English, Mandarin) in the classroom pointed to a narrowly constructed view of 
language use. Exposure to and explicit instruction about a variety of language systems might be a crucial element in program development. Paris and Ball (2009) contended that teachers should be able to understand each student's culturally and linguistically influenced styles of expression as resources and encourage the maintenance of their native languages as they support the use other new discourse patterns.

Findings in this study also revealed participants' contradictory beliefs in pronunciation of languages. They asserted the importance of perfect pronunciation of English while conveying a lack of importance in perfect pronunciation in other languages. For example, Participant $\mathrm{J}$ reported on the survey that teachers should speak correct English with students or not be directly speaking to students. Then the same participant explained in the interview that when speaking German, it was not important to use the exact pronunciation of Hochdeutsch to be understood. These beliefs indicate possible differences in how certain languages are valued. Another possible explanation suggests the importance of their personal experiences with speaking English. Many of the participants reported their parents and teachers expecting "proper" and "correct" spoken English language.

The participants in this study seem to exhibit an awareness of student diversity and monocultural policies that exclude students based on differences, but are also unsure of how the policies and norms of the mainstream culture integrate with diversity in the classroom. They seem to also be unaware that the uncertainty of integration might create a devaluing of the resources that diversity includes. Fowler (2004) explains that some children come to school unfamiliar with a teacher's indirect way of expressing herself 
and deciphering the cognitive and social patterns of school. As explained in Chapter 2 of this study, explicit instruction of these unfamiliar "codes" might be beneficial to bridge the disparities between school and home and teachers and students.

Though many of the participants in this study expressed beliefs that might be considered socially acceptable in the United States, many of the beliefs cannot be applied easily to practice. This might indicated that preservice teachers enter programs with commendable goals, but are unable to carry them out. 


\section{CHAPTER 5}

\section{IMPLICATIONS AND CONCLUSIONS}

\section{Introduction}

This study describes preservice teachers' beliefs and knowledge about language at the beginning of their teacher preparation program. Information gained in this study can be used to inform programs about what preservice teachers need to enter culturally and linguistically diverse classrooms. It is helpful to know not only the conceptual system of beliefs about language that preservice teachers bring to preparation programs, but also to know how rigidly the belief systems are held (Brown \& Cooney, 1982). Some commonly held beliefs about language may have been overlooked in the development of this study; however, the study intended to explore the relationship of beliefs and knowledge in articulation, psycholinguistics, neurolinguistics, sociolinguistics, and educational linguistics. As there is no comparison in the literature to this study regarding the five fields of exploration, the belief and knowledge categories were established based on prevailing research in individual fields.

The results of these surveys represent cross-sectional view of participants' belief and knowledge systems during one moment in time. The variability of beliefs should also be explored in future studies, as some beliefs and knowledge change over time and with exposure to diversity. This study revealed important factors that have implications for teacher preparation programs and future studies including beliefs about language acquisition, language systems, communication systems, and cultural systems as well as linguistic knowledge. Topics that generated the most variation across participant 
responses included time to learn and understand language, role of culture, importance of experiences with languages, role of grammar and vocabulary, pronunciation differences, phonological knowledge, and instructional practices.

Through interviews, the assumptions that influenced preservice teachers' initial responses became a source of reflection for the participants and information for this study. This study revealed assumptions underlying these preservice teacher beliefs. These assumptions, along with the knowledge base found from these preservice teachers' responses, provide insight for teacher preparation programs.

\section{Implications for Teacher Preparation Programs}

Findings from this study substantiate the importance of the ICC framework described in Chapter 2. All of the participants stressed the significance of mutual comprehension. Though their beliefs on how mutual comprehension might be accomplished vary, the consensus among participants reinforces the hope that these teachers will have the sensitivities and knowledge to negotiate language in the classroom. Future classrooms can be places where cultural and linguistic diversity is valued and where diverse classrooms are informed not only by cognitive principles, but also affective traits when teachers have knowledge of research in linguistics, psychology, neurology, sociology, and education. However, teacher preparation programs must also consider the incorporation of theories of ICC into their curricula to ensure that preservice teachers develop cross-cultural understandings that provide a variety of pedagogy and communication styles so students of the future will achieve at their highest potential. 
Overall, findings in this study revealed some fundamental beliefs about the language acquisition process which have varying implications for teacher preparation programs. For example, the fact that a majority of the participants believed that a student's native language and environment influences their language acquisition process could mean that these participants carefully consider the environment they create and the language they use in relation to their students' backgrounds. Additionally, the belief that it is harder to learn a language as an adolescent or adult suggests that, as secondary teachers, these participants will develop more sensitivities and awareness of the struggles that might occur with older students. However, if these secondary teachers also fail to recognize that high school students might need support at beginning levels of language acquisition then they might mistake language learning with motivation or behavior issues. These discrepancies should be addressed in teacher preparation programs.

Participants differing beliefs about how much time it takes to become proficient in language may also have implications for teacher preparation programs. Teachers in past studies have expressed beliefs that students can learn new languages virtually effortlessly and within a few years (Freeman \& Freeman, 2004; Pinker, 2007). This study has shown that, though preservice teachers still seem to believe that younger children learn new languages more easily than older people, their perception of the amount of time that it takes students to become proficient has increased from what previous studies reported. Either through personal experience or academic experience, most participants in this study have at least a basic understanding that acquiring language competency is complex. 
Findings also reveal participants had an understanding that learning a language grammatically and comprehending a language communicatively are different. Although participants revealed mixed degrees of importance in various aspects of language communication, findings also suggested that participants understand the combination of elements is a part of a complex process. These beliefs indicate that these teachers will be supportive of the language acquisition process and possibly more willing to negotiate the issues that will occur because of linguistic differences. It is important to note that participants who believe that language learning consists of mere translation or vocabulary memorization might need to adopt language use and instructional strategies that are comprehensive for successful language learning and usage. Furthermore, participants who view language diversity as a resource based on their awareness of language might reinforce positive cross-cultural communication and interaction throughout the classroom. Also, participants who actually expect students with varying proficiencies in English to proficiently understand their speech within a few years are certain to be disappointed and possibly put too much pressure on students to achieve more quickly. Furthermore, Cummins (1981) warns that educators need to be cautious about exiting children from support programs too early. He explained that students' BICS may be established, but CALP may not be. Language proficiency may not be apparent in student's oral skills. Findings in this study suggest that most of these participants share the belief in long term support that was found in the literature to be important. The varying beliefs expressed in this study indicate that teacher preparation programs must address a myriad of possibilities regarding language acquisition in language courses. 
Although participants revealed many views about language acquisition, few of the participants had distinct beliefs about the relationship between culture and language. Findings in this study are consistent with a broad range of research. Teacher preparation courses should be designed to expose preservice teachers to more opportunities to construct new concepts and sensitivities about diversity by teaching cultural and linguistic components of language. Providing experiences for preservice teachers to meaningfully interact with diverse students also plays a key role in developing an understanding about the relationship between culture and language (August \& Hakuta, 1997). Teacher preparation programs might create curricula that thread these meaningful opportunities through their programs.

Language choice was an important part of the findings in this study. Participant beliefs about language use in the classroom underscored the importance of understanding language choice. The lack of agreement between participants about the use of Standard English and other languages and dialects resembles the debate in the literature. Findings also suggested that these participants were aware that when using Standard English, specific words might create misunderstandings by implying negative connotations or inappropriate meanings. These beliefs guide the message that the students receive and, therefore, should be addressed in teacher preparation programs. Whether the language chosen by teachers literally expresses monocultural and monolingual values or implicitly reinforces the importance of using the dominant language, teacher preparation programs must highlight the issues surrounding intended teacher messages and student perception of these messages to ensure that devaluing or dismissal of student's backgrounds does not 
occur. Wood and Flodin (1990) added that each belief held by teachers influences language use and instruction and requires particular knowledge, skills, and dispositions. For example, if a teacher chooses "English only" in the classroom, students will learn that English is the valued form of expression. On the other hand, if teachers believe that it is more important to teach the mechanics of grammar and error correction methods than to encourage variation in expression, teachers will choose an approach that reinforces correct grammar such as direct instruction (Wood \& Flodin, 1990). All teachers need to be aware of different manners of language use, social class influences, cultural differences, varied instructional activities, and language opportunities that exist in diverse classrooms. Teacher preparation programs need to address the relationship between language beliefs and the power of pedagogy.

Another way for programs to foster awareness in cross-cultural interactions was introduced by Bandura (1978). He explained that one source of creating multicultural efficacy is through vicarious experiences. A vicarious experience occurs when an individual observes others and uses these observations as a source of information creating beliefs that are formed by that individual. The findings in this study indicated that participants acknowledged that their own personal experiences through work or study abroad were vital in establishing awareness, empathy, and strategies for interaction with linguistically and culturally diverse populations. Bandura (1978) advocated for creating vicarious experiences in teacher education programs that allow preservice teachers to form beliefs and knowledge that would lead to effective language use, behavior, and curriculum design for the classroom is beneficial. Future studies are needed to determine 
best practices for these experiential activities and vicarious experiences. Matching preservice teachers with mentor teachers who have demonstrated multicultural and multilingual efficacy, placing preservice teachers only in classrooms that represent diverse populations, and providing simulations that address diversity are just a few approaches to creating compensatory experiences.

Additionally, experiential activities relating to negotiating interactions with diverse populations would lead to intercultural communicative competence. The tenets of ICC could serve as a framework for designing experiences in a curriculum. Preservice teachers with limited experiences with diversity need curricula that provide vital information about language use in cross-cultural interactions and alternative approaches to teaching diverse populations. Busch (2010) suggests that it is important that experiential activities occur early in preparation programs, especially if teachers have had minimal exposure in learning L2 or contact with non-native English speakers.

Further substantiating the need for various cultural and linguistic experiences in teacher preparation is seen through research in neuroscience. Rizzolatti and FabbriDestro (2010) found that our brains use mirror neurons to learn. They explained that either by doing or by observing, mirror neurons in our brains help to decode the intentions and predict the behaviors of others creating empathy. Teacher preparation programs that design courses to help these neurons fire by using experiential activities, or vicarious experiences, or having students reflect on their personal language experiences allow preservice teachers a chance to build awareness that can powerfully impact social interactions. 
Teacher preparation programs also need to address other issues that relate to findings in neurolinguistics. New research in neurolinguistics is becoming an important area of discussion. Teachers try to change the brain everyday through their instruction. The more teachers know about how the brain learns and understands, the more successfully they can choose their language to set up the learning environment effectively. Although knowledge about how the brain processes language is often not directly transferable to classroom instruction, language is received, synthesized, created, and produced in the brain. The ability to teach in a manner that allows the brain can efficiently and effectively process and store information is crucial. Therefore, integrating neurolinguistic knowledge into teacher preparation programs would be beneficial. As neurolinguistic research develops, educators need to determine if and in what ways they should change their language.

The literature in Chapter 2 described a crucial component of ineffective teaching: a discrepancy between teachers' and students' linguistic and cultural backgrounds (Rao, 2005). This study, however, found that participants believed that a crucial component in negotiating misunderstandings in diverse classrooms was the amount of experience with cultures and languages. Conclusions drawn from this study suggest that increased empathy, respect for the learning process, and motivation to negotiate barriers increased when these preservice teachers had more exposure to cultures and languages other than their own, including experiences abroad. The experiences abroad were thought by these participants to have created a sense of understanding for what it was like to be the newcomer in a new place as well as of the specific struggles that result from language 
differences. As Participant Y explained, "I can walk in their shoes and really understanding from my own experiences what they are going through from the new language to the different ways of organizing school structures to the isolation and anxiety." Findings imply that personal experiences created empathy and a willingness to help students new to the country and school system. Because many of these participants do not share similar backgrounds, experiences, or linguistic knowledge; however, teacher preparation programs must somehow create compensatory strategies. These strategies could possibly simulate this experience for those preservice teachers that lack the exposure when entering teacher preparation programs. Teachers must have training and experiences from multiple perspectives to inform their belief systems about linguistic and cultural diversity in order to effectively teach all students (Freeman \& Freeman, 2004, Lippi-Green, 1997).

Not only is it critical to understand what beliefs educators are bringing into teacher preparation programs, it is also critical for these programs to examine the impact of their strategies and curricula on educator's beliefs and practices regarding diversity and multiculturalism (Brown, 2004). Consequently, teachers with different dominant beliefs provide strikingly different pedagogy for students (Richards \& Lockhart, 1994). If pedagogical beliefs can be positively impacted by courses dealing with diversity that allow direct experience with meaningful cross-cultural coursework, preparation program curricula should address deeper issues relating to diversity (Pohan \& Aguilar, 2001). For instance, if teachers believe that student learning is largely determined by a student's native ability and motivation, they have little reason to improve as teachers (Wood \& 
Flodin, 1990). Although teacher preparation programs typically provide individual courses in multicultural education, psychology, and linguistics, there is some work to be done in teacher preparation programs to integrate this information and assist preservice teachers in practical application of this information.

Teacher preparation programs cannot afford to ignore the preconceived beliefs and linguistic knowledge of students entering the programs. Curricula must eradicate misconceptions about language learning and use that may be based on limited knowledge. Programs must confront misunderstandings with new information (Horwitz, 1998). Teachers must show students by example and instructional practice the holistic nature of language learning.

The participants in this study were preservice teachers studying to become secondary level teachers. Findings revealed that participant's assumed that it is unimportant to have specific linguistic knowledge, including detailed knowledge of the language acquisition process or knowledge of decoding and phonology. Findings indicated that as secondary teachers they believed that they would not serve students that did not have basic knowledge and skills of the English language. Findings indicated the belief that "older students already have the knowledge they need to progress with using English. They just need some support.” Gandara, Maxwell-Jolly, and Driscoll (2005) explained that the assumption that learning English is a problem faced by early childhood and elementary teachers has created barriers in secondary teachers' pedagogical knowledge that is necessary to teach ELLs. 
Wren (2002) found that some students in high school are below basic levels of literacy and language proficiency required to meet academic achievement. Wren cited three main teacher beliefs that affected their instruction including 1) teachers did not see themselves as reading teachers, 2) teachers did not realize that students came to high school with only elementary level language skills either because they struggled to learn or recently moved to the United States, and 3) teachers stated that they did not receive training in basic linguistic and literacy instruction. Despite the understanding that language learning becomes more complex for older students because of the brain's learning capabilities, Sousa (2011) explained that students with low language proficiencies in upper grades have not been researched sufficiently. Therefore, the gap in teacher knowledge of foundational language use must be filled and this serves to inform teacher preparation programs. Preservice teachers, elementary and secondary alike, need basic linguistic knowledge. However, the participants in this study did not recognize their need for linguistic knowledge.

Preservice teachers might not explicitly consider how their language use and students' linguistic needs impact student learning (Ball, 2000). According to Berry (2002), teachers have to act on what they know. However, what if that knowledge is incomplete? As stated throughout this study, teachers have significant impact on student learning (Smith, 1988). Teachers need to enter culturally and linguistically diverse classrooms with language awareness. Teacher preparation programs must support development of language beyond superficial understandings of the impacts of language use on diverse populations. 
Findings in this study indicated a lack of linguistic knowledge overall. According to Kintner-Duffy (2011), teachers' knowledge was more predictive of classroom practices with children from culturally and linguistically backgrounds than were teacher belief systems. She found that the more linguistic knowledge a teacher held, the more likely that teacher was to adapt practices to accommodate all children. Teacher education with a focus on linguistic knowledge can foster school cultures that are inclusive and responsive to linguistic diversity. Furthermore, programs grounded in linguistic principles aid educators in developing pedagogical strategies and language choice that are effective with diverse student populations.

\section{Implications for Future Studies}

Future research is needed to assist teacher education programs to better prepare all candidates to work with the increasing populations of ELLs (August \& Hakuta, 1997). Along with the studies mentioned in Chapter 4 for further exploration, the following studies are recommended. Future studies might investigate the relationship of affective traits and language. In this study, participants never spoke of affective traits that influence language use and understanding. Further studies should investigate what role emotion, value systems, and motivation play in language use and teaching culturally and linguistically diverse classrooms with regard to research findings in articulation, psycholinguistics, neurolinguistics, sociolinguistics, and educational linguistics.

Exploration of preservice teacher language behavior was outside the scope of this study. However, further research to confirm alignment of said beliefs with behavior is necessary. It was also not the scope of this study to evaluate how preservice teacher 
language beliefs and knowledge about language transfer into the classroom. Future studies might use longitudinal methods to evaluate how stated language beliefs and knowledge align with actual behavior and if beliefs or knowledge have a greater impact on behavior. Future studies might also consider how a teacher's talk is perceived by students in a diverse classroom. Studies of the negotiation of language use between teacher and student might provide informative data in the understanding of linguistic issues that occur in diverse classrooms. These studies are essential to ensuring quality teaching that leads to positive outcomes for all students.

Future studies might also include comparisons between monolingual and multilingual teachers. Findings in this study indicated no significant difference in language beliefs and linguistic knowledge between these two groups, except with regard to standard language use and phonology. These findings were contrary to the findings of other studies. Therefore, in the future, research exploring similarities and differences in overall linguistic knowledge and specific linguistic knowledge such as rhyming and how that translates to language use in classrooms could be beneficial in establishing understandings about pedagogical choices and language manipulation, possibly shifting the paradigm toward deeper cultural and linguistic understandings.

\section{Conclusions}

It is vital for educators to understand the function language can play in either assisting or inhibiting the education of all students despite their cultural or linguistic backgrounds (Ovando, (1997). In the United States, policies have been established that demand equal education opportunities regardless of language. To create equality, Lippi- 
Green (1997) contended that many states have established guidelines that require teachers to "demonstrate excellent skills of pronunciation and grammar" and states such as New York that require pronunciation and accent tests for prospective teachers and accent reduction classes for students (p. 122). Though participants in this study seem to agree that using Standard English correctly is beneficial, findings also concluded that the opportunity for celebrating all cultures and languages represented in the classroom was possible. According to Hymes (1981), “One's language affects one's chances in life, not only through accent, but also through action" (p. vii). Policy creation often becomes a matter of language control (Brown, 2007). Policy makers could benefit from knowledge that a linguist could offer though this knowledge is rarely sought or is dismissed quickly (Lippi-Green, 1997). For example, the influence of a linguist's definition of communicative competence and what this competence means for classroom practice are rarely examined. Policy makers and teacher preparation programs must consider the effects of language use when designing policies and curricula. Creating policies that provide cultural and linguistic support for teachers as well as students, support for family involvement by bridging language and cultural differences, support for addressing the shortage of qualified teachers to work with diverse populations, and support with funding issues can also increase educational opportunities for all students.

Language policies definitively affect teaching. A large portion of teaching requires spoken language. This spoken language is often taken for granted. In this study, participants made comments such as "that [they] have been to college," "have a large vocabulary," and even "[were] English majors," language use was not a concern, "but 
behavior management, content knowledge, and instruction methods are more prevalent issues." Beliefs such as these leave open the possibilities of language use that devalues students or prevents access to learning. Viiri and Saari (2006) contended that in addition to teacher education programs teaching preservice teachers explicitly how to use their content knowledge to plan lessons, teaching preservice teachers to use their knowledge of language to explicitly plan "talk types or talk patterns" for different situations and diverse populations would allow teachers to master various methods of interacting with students.

Moreover, Richards and Lockhart (1994) contended that no matter what instructional strategies or methods are used in the classroom, teachers must give instructions, explain activities, clarify procedures, and check student's understanding. Also referred to as teacher talk, a teacher's language use must be modified to be easily understood by all students to provide support for language comprehension and language production. Teachers also need to think about the words they choose because the interpretation of one's language choice varies with each individual.

We must be careful about providing or denying access to opportunities such as schooling and jobs that depend on ways of using language because we do not understand how our own beliefs manifest themselves in our behavior or because of our lack of knowledge about language. Beliefs vary with experience, age, knowledge, and culture, among other variables; awareness and discussion can serve to counteract or reinforce beliefs. An evaluation of the interrelatedness of beliefs, knowledge, and behavior is an essential in understanding. 
Teachers, having been students themselves, are influenced by the teaching styles and methods that they have been exposed to throughout their lives. Findings in this study reveal that belief systems are built around this exposure and their experiences with diversity. Teacher beliefs also inform what kinds of new knowledge teachers are willing to receive and how they apply it to the classroom (Van Driel, Bulte, \& Verloop, 2007). Teachers who are unaware of their beliefs and knowledge about language cannot put these beliefs into action in an intentional way in diverse classrooms (Parajes, 1992).

It is not enough to use prescribed curriculum or blame others for unmet needs, teachers must work to ensure that the diverse needs of the students are met. Teachers must be provided with the tools to achieve this complex task. With classrooms working in connection with communities, negotiation between cultures and languages can create positive experiences that establish respect.

Isaih Berlin (as cited in Noddings, 2005, p. 9) advised caution when deciding what is in "others"” best interest. Ovando (1997) explained that one way to establish curricular approaches that meet the unique needs of all students is to support the idea of creating bridges between the world of the language-minority student's home and school. He stated that these bridges will produce positive cognitive, linguistic, and cultural outcomes. He further explained that teachers need to have an awareness of how language is used in the student's home communities to extend the bridge for students. Orr (1994) further contended that we cannot state that we know something until we can state that we understand the effects of this knowledge on the people and communities to which the 
knowledge applies. What is language, what does it mean to students, and what is appropriate pedagogy?

According to Darling-Hammond and Baratz-Snowden (2007), we now know more about how people learn and how to teach effectively. They further stated, however, "that much of this knowledge is only haphazardly available those who most need it to do their work - the teachers who today are charged with enabling students to reach the highest standards of accomplishment" (p.113). They also stated that the complexity of teaching requires knowledge in navigating cultural, linguistic, socio-economic, and developmental differences in students. Juggling academic, social, and political goals requires informed decision making skills. It requires integrating knowledge for practical application.

In sum, teachers should be grounded in the contexts of their classrooms. Most importantly, they should have knowledge of their students and the communities in which they teach. In addition, teachers should have knowledge of other teachers in the school, district philosophies, and resources available. Lastly, teachers should have content knowledge and linguistic knowledge to negotiate language barriers and provide effective instruction. It is crucial to integrate knowledge to allow all students access to teachers' language use. Integrating findings from various fields is complex, but necessary for effectively teaching the whole student, not just isolated parts. Incorporating cognitive functions from the perspectives of psycholinguistics, neurolinguistics, sociolinguistics, and educational linguistics, creates a broader framework that provides teachers with more methods of overcoming misunderstandings and preventing misdiagnosis in evaluations. 
Language is rooted in the mind and used based on real-time processing, social interactions, and instructional choices in the classroom. Therefore, language processes should be explored and understood by integrating theories and methods from all disciplines involved in studying the mind and brain (Walenski \& Ullman, 2005). For example, Walenski and Ullman (2005) found that a combination of research findings in the fields of psycholinguistics, neurolinguistics, and educational linguistics conclude that ability to learn language in declarative memory improves throughout childhood and plateaus in adolescence. These findings provide evidence for the ease of learning language as a young child with increasing difficulty through adulthood. It also provides evidence for the ability to learn all aspects of language while young and that the ability atrophies with age making the native pronunciation more difficult when learning language later in life. This knowledge carries different implications for the elementary or secondary teacher.

Another relevant example of integration involves the fields of sociology and neurology. Merging research in these fields has produced evidence of neuroplasticity occurring throughout life. Neuroplasticity occurs when new experiences reshape the organization of the brain. Recent research has found that to continue learning the brain creates new neurons and connections and this ability is critical in acquiring new knowledge and skills throughout life (Lee \& Hillis, 2008). Lee and Hillis (2008) also contended that neuroplasticity can be stimulated by factors such as positive new experiences, pharmacological interventions, or language therapy which can even compensate for language deficits. For years, researchers in the field of sociology have reported the impacts of new experiences on language development, socialization, and 
world views (Malinowski, 1944; Ochs \& Schieffelin, 1982). Current research in neurology is affirming and enhancing understandings about the importance of experiences on personal belief systems, knowledge, and behavior that sociologists have held for some time. Additionally, teachers that reflect on communicative interactions provide the brain with more neural patterns that impact their ability to use language effectively.

Converging evidence that supports similar findings about language is beneficial in designing curricula or exploring effective methods of language use with diverse populations. In exploration of the overlapping concepts of language use such as articulation, word recognition, neurological processing, social interaction, and education system acts, educators might better understand ways in which people communicate. Margaret Wheatley (2006) wrote about the importance of creating awareness through the world of interconnectedness. She explained that when interacting with diverse people, seeing them as whole systems is more beneficial than looking at isolated parts. This integration broadens the conversations about the relationship between preservice teacher's belief and knowledge about language and effective language use in diverse classrooms.

As no one model or curricula accounts for all backgrounds and learning modalities, a conceptual model of various methods of language use could be developed to enhance teacher preparation programs and strengthen teachers' competencies. This study used interdisciplinary knowledge and belief systems of language and provides an understanding of the types of belief systems preservice teachers have when entering preparation programs. Further development of research that uses integrative concepts 
will strengthen teacher preparation programs and help preservice teachers to critically examine their role and influence within diverse classrooms. 


\section{REFERENCES}

Abutalebi, J., Miozzo, A., \& Cappa, S. F. (2000). Do subcortical structures control "language selection" in polyglots? Evidence from pathological language mixing. Neurocase, 6, 51-56.

ACTFL Proficiency Guidelines. (2011). Hastings-on-Hudson, NY: American Council on the Teaching of Foreign Languages.

Agar, M. (1994). Language shock: Understanding the culture of conversation. New York, NY: HarperCollins.

Allington, R. (1984, May). Policy constraints and effective compensatory reading instruction: A review. Paper presented at the annual meeting of the International Reading Association, Atlanta, GA.

American Speech-Language-Hearing Association. (2005). Standards and implementation procedures for the certificate of clinical competence in speech-language pathology. Retrieved from http://www.asha.org/certification/slp_standards

Anderssen, L.G. (1998). Some languages are harder than others. In L. Bauer \& P. Trudgill (Eds.), Language myths. New York, New York: Penguin Putnam, Inc.

Andrews, A. (2007). Teacher language awareness. Cambridge, United Kingdom: University Press.

Artiles, A. J., Barreto, R. M., \& Pena, L. (1998). Pathways to teacher learning in multicultural contexts. Remedial and Special Education, 19(2), 70-90. 
Auer, P. \& Wei, L. (2008). Introduction: Multilingualism as a problem? Monolingualism as a problem? In P. Auer \& L. Wei (Eds.), Handbook of multilingualism and multilingual communication. Berlin, Germany: Walter de Gruyter GmbH \& Co.

August, D., Hakuta, K., \& National Research Council. (1997). Improving schooling for language-minority children: A research agenda. Washington, DC: National Academy Press.

Ball, A. (2000). Empowering pedagogies that enhance the learning of multicultural students. Teachers College Record, 102(6), 169-202.

Baker, J. (2002). Trilingualism. In L.Delpit \& J. Kilgour Dowdy (Eds.), The skin that we speak: Thoughts on language and culture in the classroom (pp. 49-62). New York, NY: New York Press.

Baker, S. A., Kovelman, I., Bialystok, E., \& Petitto, L. A. (2003). Bilingual children's complex linguistic experience yields a cognitive advantage. Published Abstracts of the Society for Neuroscience, 1506.

Barcelos, A. M. (2006). Researching beliefs about SLA: A critical review. In P. Kalaja \& A.M. F. Barcelos (Eds.), Beliefs about SLA: New research approaches (pp. 7-33). New York, NY: Springer Science + Business Media, LLC.

Bernstein, B. (1975). Class, codes, and control (Vol. 3). London, UK: Routledge Kegan Paul.

Bernstein Ratner, N., Berko Gleason, J., \& Narasimhan, B. (1998). An introduction to psycholinguistics: What do language users know? In J. Berko Gleason, N. 
Bernstein Ratner (Eds.), Psycholinguistics (pp. 1-49). Orlando, FL: Harcourt Brace.

Berry, R. A., \& Kim, N. (2008). Exploring teacher talk during mathematics instruction in an inclusion classroom. The Journal of Educational Research, 101(6), 363-377.

Bialystok, E. (2007). Language acquisition and bilingualism: Consequences for a multilingual society. Applied Psycholinguistics, 28, 393-397.

Bialystok, E., Craik, F. I. M., \& Ruocco, A. C. (2006). Dual-modality monitoring in a classification task: The effects of bilingualism on aging. The Quarterly Journal of Experimental Psychology, 59, 1-16.

Blommaert, J. (2007). Linguistic diversity: Africa. In M. Hellinger \& A. Pauwels (Eds.), Handbook of language and communication: Diversity and change (Vol. 9, pp. 123-149). Berlin, Germany: Mouton de Gruyter.

Boas, F. (1911). Race and language. In R.B. Dixon, P.E. Goddard, W. Jones, T. Michelson, J. R. Swanton, \& W. Thalbitzer, (Eds.), Handbook of American Indian languages (pp. 5-14). Washington. DC: Government Printing Office.

Bornkessel, I., \& Schlesewsky, M. (2006). The extended argument dependency model: A neurocognitive approach to sentence comprehension across languages. Psychological Review, 113(4), 787-821.

Bornkessel-Schlesewsky, I. \& Friederici, A. D. (2009). Neuroimaging studies of sentence and discourse comprehension. In Gaskell, M. G. (Ed.), The Oxford handbook of psycholinguistics (pp. 407-424). Oxford, UK: Oxford University Press. 
Bornkessel-Schlesewsky, I. \& Schlesewsky, M. (2009). Processing syntax and morphology: a neurocognitive perspective. New York, NY: Oxford University Press.

Bourdieu, P. (1990). In Other Words: Essays Towards a Reflexive Sociology. Cambridge: Polity Press.

Boyer, E. L. (1990). Scholarship reconsidered: Priorities of the professoriate. Princeton, NJ: The Carnegie Foundation for the Advancement of Teaching.

Breedin, S., \& Saffran, E. (1999). Sentence processing in the face of semantic loss: A case study. Journal of Experimental Psychology: General, 128, 547-562.

Brown, K. M. (2004). Assessing preservice leaders' beliefs, attitudes, and values regarding Issues of diversity, social justice, and equity: A review of existing measures. Equity \& Excellence in Education, 37(4), 332-342.

Buckingham, H. W., \& Christman, S. C. (2008). Disorders of phonetics and phonology. In B. Stemmer \& H. A. Whitaker (Eds.), Handbook of the neuroscience of language (pp. 127-136). London, UK: Elsevier.

Byram, M. (1997). Teaching and assessing intercultural communicative competence. Clevedon, UK: Multilingual Matters.

Caramazza, A., Berndt, R. S., Basili, A. G., \& Koller, J. J. (1981). Syntactic processing deficits in aphasia. Cortex, 17(3), 333-348.

Carroll, J. B. (1964). Language and thought. Englewood Cliffs, NJ: Prentice-Hall. Chambers, J. K. (1995). Sociolinguistic theory: Linguistic variation and it social significance. Oxford, UK:: Blackwell. 
Chaudron, C. (1985). A method for examining the input/intake distinction. In S. Gass \& C. Madden (Eds.), Input in second language acquisition (pp. 285-300). Rowley, MA: Newbury House.

Chen, G. M. (1993, November). Dimensions of intercultural communication competence. Paper presented at the annual convention of Speech Communication Association, Miami Beach, FL.

Chomsky, N. (1959). A review of B. F. Skinner's Verbal Behavior. Language, 35, 26-58. Chomsky, N. (1968). Language and the mind. Psychology Today, 1(9), 48-68.

Clark, J. L. D. (1967). Empirical studies related to the teaching of French pronunciation to American students (Report BR-5-1093). Cambridge, MA: Harvard University, Laboratory for Research in Instruction.

Colombo, J. A., \& Bundy, R .S. (1981). A method for the measurement of infant auditory selectivity. Infant Behavior and Development, 4, 219-223.

Costa, A., Alario, F.X., \& Sebastian-Galles, N. (2009). Cross-linguistic research on language production. In Gaskell, M. G. (Ed.), The Oxford handbook of psycholinguistics (pp. 531-546). Oxford, UK: Oxford University Press.

Costa, A., \& Caramazza, A. (1999). Is lexical selection in bilingual speech production language specific? Further evidence from Spanish-English and English-Spanish bilinguals. Bilingualism: Language and Cognition, 3, 231-243.

Coward, A. (1990). Pattern thinking. New York, NY: Praeger.

Creswell, J. W. (2008). Educational research: Planning, conducting, and evaluating quantitative and qualitative research ( $3^{\text {rd }}$. ed.). Upper Saddle River, NJ: Pearson Prentice Hall. 
Cruse, D. A. (1986). Lexical semantics. Cambridge, UK: Cambridge University Press.

Cummins, J. (1981). The role of primary language development in promoting educational success for language minority students. In California State University (Ed.) Schooling and language minority students: A theoretical framework (pp. 3-49). Los Angeles, CA: Evaluation, Dissemination, and Assessment Center, California State University.

Curtin, S., \& Werker, J. F. (2009). The perceptual foundations of phonological development. In Gaskell, M. G. (Ed.), The Oxford handbook of psycholinguistics (pp. 579-600). Oxford, UK: Oxford University Press.

Cutler, A., \& Otake, T. (1999). Pitch accent in spoken word recognition in Japanese. Journal of the Acoustical Society of America, 105, 1877-1888.

Deardorff, D. K. (2006). Identification and assessment of intercultural competence as a student outcome of internationalization. Journal of Studies in International Education, 10, 241-266.

Dell, G.S. (1986). A spreading activation theory of retrieval in sentence production. Psychological Review, 93, 283-321.

Delpit, L. (1995). Other people's children: Cultural Conflict in the Classroom. New York, NY: The new Press.

Dewey, J. (1933). How we think. Lexington, MA: DC: Heath.

Dijkstra, T. (2009). The multilingual lexicon. In Gaskell, M. G. (Ed.), The Oxford handbook of psycholinguistics (pp. 251-265). Oxford, UK: Oxford University Press. 
Dunkin, M. J., \& Biddle, B. J. (1974). The study of teaching. New York, NY: Holt, Rinehart, \& Winston.

Edl, H. M., Jones, M. H., \& Estell, D. B. (2008). Ethnicity and English proficiency: Teacher perceptions of academic and interpersonal competence in European American and Latino students. School Psychology Review, 37(1), 38-45.

Ellis, R. (1999). Learning a second language through interaction. Philadelphia, PA: John Benjamins.

Elman, J., Bates, E.A., Johnson, M., Karmiloff-Smith, A., Parisi, D., \& Plunkett, K. (1997). Rethinking innateness: A connectionist perspective on development. Cambridge, MA: MIT Press.

Ferguson, R. F. (1998). Teachers' perceptions and expectations and the Black-White test scores gap. In C. Jencks \& M. Phillips (Eds.), The Black-White test score gap (pp. 273-317). Washington DC: Brookings Institution Press.

Fill, A. (2007). Language contact, culture, and ecology. In M. Hellinger \& A. Pauwels (Eds.), Handbook of language and communication: Diversity and change (pp. 176-207). Berlin, Germany: Mouton de Gruyter.

Fillmore, L. W. (1985). Teacher talk that works. In S. Gass \& C. Madden (Eds.), Input in second language acquisition (pp. 17-50). Rowley, MA: Newbury House.

Fillmore, L. W., Snow, C. E., \& Educational Resources Information Center (U.S.). (2000). What teachers need to know about language. Washington, DC: U.S. Department of Education, Office of Educational Research and Improvement, Educational Resources Information Center. 
Fowler, C. A. (2009). Speech production. In Gaskell, M. G. (Ed.), The Oxford handbook of psycholinguistics (pp. 489-501). Oxford, UK: Oxford University Press.

Fox, R. A., \& Unkefer, J. (1985). The effect of lexical status on the perception of tone. Journal of Chinese Linguistics, 13, 69-90.

Fraenkel, J., \& Wallen, N. (2003). How to design and evaluate research in education $\left(5^{\text {th }}\right.$ ed.). Boston, MA: McGraw Hill.

Frauenfelder, U. H., Scholten, M., \& Content, A. (2001). Bottom-up inhibition in lexical selection: Phonological mismatch effects in spoken word recognition. Language and Cognitive Processes, 16, 583-607.

Freeman, D. E., \& Freeman, Y. S. (2004). Essential linguistics: What you need to know to teach. Portsmouth, NH: Heinemann.

Freeman, W. (1995). Societies of brains. Hillsdale, NJ: Erlbaum.

Friederici, A. D. (2005). Neurophysiological markers of early language acquisition: from syllables to sentences. TRENDS in Cognitive Sciences, 9(10), 481-488.

Furman, G. (1998). Postmodernism and community in schools: Unraveling the paradox. Educational Administration Quarterly, 34(3), 298-328.

Gallios, C., Watson, B., \& Brabant, M. (2007). Attitudes to language and communication. In M. Hellinger \& A. Pauwels (Eds.), Handbook of language and communication: Diversity and change (Vol. 9, pp. 595-618). Berlin, Germany: Mouton de Gruyter.

Gandara, P., Maxwell-Jolly, J., \& Driscoll, A. (2005). Listening to teachers of English language learners: A survey of California teachers' challenges, experiences, and 
professional development needs. The Center of Future teaching and Learning. Retrieved from http://www.cftl.org/documents/2005/listeningforweb.pdf

Gardner, H. (1991). The unschooled mind: How children think \& how schools should teach. New York, NY: BasicBooks.

Gee, P. (2001). Educational linguistics. In M. Arnoff \& J. Rees-Miller (Eds.), Handbook of linguistics (pp. 647-663). Malden, MA: Blackwell.

Geertz, C. (1983). Local knowledge: Further essays in interpretive anthropology. New York, NY: BasicBooks.

Gekoski, W. L. (1980). Language acquisition context and language organization in bilinguals. Journal of Psycholinguistics, 9, 431- 458.

Genesee, F. (2000). Brain research: Implications for second language learning. Santa Cruz, CA: Center for Research on Education Diversity and Excellence..

Gentner, D. (1981). Some interesting differences between nouns and verbs. Cognition and Brain Theory, 4, 161-178.

Giles, H., \& Robinson, W.P. (1990). The handbook of language and social psychology. Chichester, UK: Wiley.

Gitomer, D. H., Andal, J., \& Davison, D. (2005). Using data to understand the academic performance of English language learners. Policy Issues. Number 21. Learning Point Associates/North Central Regional Educational Laboratory (NCREL.). Godley, A, Sweetland, J., Wheeler, R. S., Minnici, A., \& Carpenter, B. D. (2006). Preparing teachers for dialectically diverse classroom. Educational Research, 35(8), 30-37. 
Gonzalez, J. (1984). Language processing and bilingualism. Retrieved from http://www.eric.ed.gov/PDFS/ED253069.pdf

Gow, D. W. (2001). Assimilation and anticipation in continuous spoken word recognition. Journal of Memory and Language, 45, 133-159.

Graduate Teacher Education Program. (n.d.) Graduate Teacher Graduate Program (GTEP). Retrieved March 3, 2011, from http://www.pdx.edu/ci/graduate-teachereducation-program.

Graff, G. (2003). Clueless in academe: How schooling obscures the life of the mind. New Haven, CT: Yale University Press.

Greene, J. C., \& Caracelli, V. J. (1997). Advances in mixed method evaluation: The challenges and benefits of integrating diverse paradigms. San Francisco, CA: Jossey-Bass.

Gumperz, J. J., \& Hernandez-Chavez, E. (1972). Bilingualism, bidialectalism, and classroom interaction. In C.B. Cazden, V.P. John, \& D. Hymes (Eds.), Functions of language in the classroom (pp. 84-108). New York, NY: Teachers College Press.

Halliday, M. A. K. (1975). Learning how to mean: Explorations in the development of language. London, UK: Edward Arnold.

Harris, S., Sheth, S.A., \& Cohen, M.S. (2008). Functional neuroimaging of belief, disbelief, and uncertainty. Annals of Neurology, 63(2), pp. 14-147.

Harste, J.C, Woodward, V.A., \& Burke, C.L. (1984). Language stories and literacy lessons. Portsmouth, NH: Heinemann. 
Hatch, E. \& Wagner-Gough, J. (1975). The importance of input data in second language acquisition. Language learning, 25(2), p. 297-311.

Healy, J. (1990). Endangered minds: Why our children can't think. New York, NY: Simon \& Schuster.

Heath, S. B. (1986). Taking a cross-cultural look at narratives. Topics in Language Disorders, 7 (1), 89-94.

Heath, S. B. (1983). Way with words: Language, life, and work in communities and classrooms. Cambridge, UK: Cambridge University Press.

Hoff, E. (2006). How social contexts support and shape language development. Developmental Review, 26, 55-88.

Hopper, R., \& Naremore, R.C. (1973). Children's speech: A practical introduction to communication development. New York, NY: Harper \& Row.

Horwitz, B., \& R. J. S. Wise. (2008). PET research of language. In B. Stemmer \& H.A. Whitaker (Eds.), Handbook of the neuroscience of language (pp. 71-80). London, UK: Elsevier.

Hudson, R. (2010). Linguistic theory. In B. Spolsky \& F. M. Hult (Eds.), Handbook of educational linguistics (p. 53-65). Malden, MA: Blackwell Wiley.

Hymes, D. (1972). Introduction. In C. B. Cazden, V. P. John, \& D. Hymes, (Eds.), Functions of language in the classroom (pp. v-lvii). New York, NY: Teachers College Press.

Jackendoff, R. (1983). Semantics and cognition. Cambridge, MA: MIT Press. 
Jenkins, J. (2009). English as a lingua franca: interpretations and attitudes. World Englishes, 28(2), 200-207.

Jensen, E. (1998). Teaching with the brain in mind. Alexandria, VA: Association for Supervision and Curriculum Development.

Jusczyk, P. W. (1997). The discovery of spoken language. Cambridge, MA: MIT Press.

Justice, P. W. (2004). Relevant linguistics: An introduction to the structure and use of English for teachers. Stanford, CA: Center for the Study of Language Institution.

Kachru, Y. (2008). Language variation and corpus linguistics. World Englishes, 27(1), 18.

Kadeghe, M. (2000). The implications of bilingual education in learning and teaching: The case of Tanzanian secondary schools. Unpublished doctoral dissertation, University of Dar es Salaam.

Karzon, R. G. (1985). Discrimination of polysyllabic sequences by one-to-four-monthold infants. Journal of Experimental Child Psychology, 39, 326-342.

King, P. M., \& Baxter-Madgola, M. (2005). A developmental model of intercultural maturity. Journal of College Student Development, 46(6), 571-592.

Kintner-Duffy, V. (2011). "Everybody's Different and the Same": An inquiry into early childhood teachers' beliefs, knowledge, and practices in relation to children from culturally diverse backgrounds. (Doctoral Dissertation). Retrieved from http://proquest.umi.com.proxy.lib.pdx.edu. (AAT 3457638)

Kohl, H. (2002). Topsy-turvies: Teacher talk and student talk. In L.Delpit \&J. Kilgour Dowdy (Eds.), The skin that we speak: Thoughts on language and culture in the classroom (pp. 145-162). New York, NY: New York Press. 
Kono, N. (2001). Language orientation: Case study of a Japanese- as-a-foreignlanguage-classroom. Retrieved from ProQuest Digital Dissertation. (AAT 3010267).

Kovacs, A. M., \& Mehler, J. (2009). Cognitive gains in 7-month-old bilingual infants. Proceedings of the National Academy of Sciences, 106(16), 6556-6560.

Krishnan, A., \& Gandour, J. T. (2009). The role of auditory brainstem in processing linguistically-relevant pitch patterns. Brain and Language, 110(3), 135-148.

Krashen, S. (1982). Principles and practice in second language acquisition. New York, NY: Pergamon Press.

Labov, W. (1982). Building on empirical foundations. In W.P. Lehmann \& Y. Malkiel, (Eds.), Perspectives on historical linguistics (pp. 17-92). Amsterdam, Netherlands: John Benjamins.

Labov, W. (1994). Principles of linguistic change: Internal factors. Maldon, MA: Blackwell.

Ladson-Billings, G. (1994). The dreamkeepers: Successful teachers of African American children. San Francisco, CA: Jossey-Bass Publishers.

Larson-Freeman, D. (1985). State of the art on input in second language acquisition. In S. Gass \& C. Madden (Eds.), Input in second language acquisition (pp. 433-444). Rowley, MA: Newbury House. 
Lasky, R. E., Syrdal-Lasky, A., \& Klein, R. E. (1975). VOT discrimination by four-tosix-and-a-half-month-old infants from Spanish environments. Journal of Experimental Child Psychology, 20, 215-225.

Laver, J. (1968). Voice quality and indexical information. British Journal of Disorders of Communication, 3, 43-54.

Laver, J. \& Trudgill, P. (1979). Phonetic and linguistic markers in speech. In K.R. Scherer, \& H. Giles (Eds.), Social markers in speech (pp. 1-32). Cambridge, UK: Cambridge University Press.

Lee, A.W., \& Hillis, A.E. (2008). The pharmacological treatment of aphasia. In B. Stemmer \& H.A. Whitaker (Eds.), Handbook of the neuroscience of language (pp. 407-415). London, UK: Elsevier.

Lehiste, I. (1972). The timing of utterances and linguistics boundaries. Journal of Acoustic Society of America, 51, 2018-2024.

Lenski, S.D., Wham, M.A., \& Griffey, D.C. (1998). Literacy Orientation Survey: A Survey to Clarify Teachers' Beliefs and Practices. Reading Research and Instruction, 37 (3), 217-236.

Levi-Strauss, C. (1968). Structural anthropology. London, UK: Penguin Press.

Lindfors, J. W. (1991). Children's language and learning ( $2^{\text {nd }}$ ed.). Needham, MA: Allyn and Bacon.

Lindholm, K. J., \& Padilla, A. M. (1978). Language mixing in bilingual children. Journal of Child Language, 5, 334-346.

Lippe-Green, R. (1997). English with an accent. New York, NY: Routledge. 
Long, M. (1985). Input and second language acquisition theory. In S. Gass \& C. Madden (Eds.), Input in second language acquisition (pp. 377-393). Rowley, MA: Newbury House.

MacNamara, J., Krauthammer, M., \& Bolgar, M. (1968). Language switching in bilinguals as a function of stimulus and response uncertainty. Journal of Experimental Psychology, 78, 208-215.

Malinowski, B. (1944). A scientific theory of culture, and other essays. Chapel Hill, NC: The University of North Carolina Press.

Mariano, C. (2001). Case study: The method. In P. Marshall (Ed.), Nursing research: A qualitative perspective (pp. 359-383). Sudbury, MA: Jones and Bartlett.

Marshall, C., \& Rossman, G. B. (2011). Designing qualitative research. Thousand Oaks, CA: Sage .

Marslen-Wilson, W., \& Zwitserlood, P. (1989). Accessing spoken words: The importance of word onsets. Journal of Experimental Psychology: Human Perception and Performance, 15, 576-585.

Martin, R. C., Vuong, L. C., \& Crowther, J. E. (2009). Sentence-level deficits in aphasia. In M. G. Gaskell (Ed.), The Oxford handbook of psycholinguistics (pp.425-439). Oxford, UK: Oxford University Press.

Maxwell, J. A. (2005). Qualitative research design. Thousand Oaks, CA: Sage. McBride-Chang, C,. Shu, H., Ng, J.Y.W., Meng, X., Penney, T. (2007). Morphological structure awareness, vocabulary, and reading. In R. K. Wagner, A. E. Muse, and K. R. Tannenbaum (Eds.), Vocabulary acquisition: Implications for reading comprehension (pp. 104-122). New York: New York: The Guilford Press. 
McQueen, J. M. (2009). Eight questions about spoken word recognition. In M. G. Gaskell (Ed.), The Oxford handbook of psycholinguistics (pp. 37-53). Oxford, UK: Oxford University Press.

McWhorter, J. H. (2001). The power of Babel. New York, NY: Henry Holt.

Meier, D. (2011). Proceedings from KDP $48^{\text {th }}$ Biennial Convocation 2011: Promote Democracy in Your Classroom. Indianapolis, IN: Author.

Merriam, S. B. (1998). Qualitative research and case study applications in education. San Francisco, CA: Jossey-Bass.

Miller, G. A., \& Fellbaum, C. (1991). Semantic networks of English. Cognition, 41, 197229.

Milroy, J., \& Milroy, L. (1999). Authority in language: Investigating standard English. London, UK: Routledge.

Moats, L. C. (1994). The missing foundation in teacher education: Knowledge of the structure of spoken and written language. Annals of Dyslexia, 44, 88-102.

Moll, L.C., Saez, R., \& Dworin, J. (2001). Exploring biliteracy: Two student case examples of writing as a social practice. The Elementary School Journal, 101(4), 221-246.

Mornay, D. P., de, \& Funnell, E. (2000). Semantic representation and ease of predication. Brain and Language, 73, 92-119.

Moss, H. E., Tyler, L. K., \& Taylor, K. I. (2009). Conceptual structure. In M. G. Gaskell (Ed.), The Oxford handbook of psycholinguistics (pp.119-139). Oxford, UK: Oxford University Press.

Nabokov, V. (1955). Lolita. New York, NY: Random House. 
National Association for the Education of Young Children. (2009). NAEYC Standards for Early Childhood Professional Preparation. Retrieved on October 15, 2011 from http://oldweb.naeyc.org/faculty/pdf/2001.pdf

National Center for Educational Statistics. (2003). The Nation's report card. Retrieved November 12, 2009, from http://nces.ed.gov/nationsreportcard

National Center for Research on Cultural Diversity and Second Language Learning. (2002). The standards for effective teaching. Retrieved on October 14, 2011 from http://www-bcf.usc.edu/ cmmr/crede.html

National Council of Teachers of English (1993). Doublespeak here and there. Quarterly Review of Doublespeak (April), 1-7.

Nickels, L. (2008). The hypothesis testing approach to the assessment of language. In B. Stemmer \& H.A. Whitaker (Eds.), Handbook of the neuroscience of language (pp. 13-21). London, UK: Elsevier.

Nieto, S., \& Bode, P. (2008). Affirming diversity: The sociopolitical context of multicultural education. Boston, MA.: Pearson.

Nocon, H., \& Cole, M. (2009). Relating diversity and literacy theory. In L. M. Morrow \& R. Rueda (Eds.), Handbook of research on literacy and diversity (pp. 13-31). New York, NY: Guilford Press.

Noddings, N. (2005). Global Citizenship: Promises and Problems. In N. Noddings (Ed.), Educating Citizens for Global Awareness (pp. 1-21). New York, NY: Teachers College Press.

Norris, D. (1994). Shortlist: A connectionist model of continuous speech recognition. Cognition, 52, 189-234. 
Norton, E., Baker, S., \& Petitto, L. A. (2003, July). Bilingual infants' perception of handshapes in American Sign Language. Poster presented at the University of Pennsylvania Institute for Research in Cognitive Science summer workshop, Philadelphia, PA.

Ottenheimer, H. J. (2009). The anthropology of language: An introduction to linguistic anthropology. Belmont, CA: Wadsworth.

Ovando, C. J. (1997). Language diversity and education. In J. Banks \& C.A.M. Banks (Eds.), Multicultural education: Issues and perspectives. Needham Heights, MA: Allyn and Bacon.

Page, S. E. (2007). The difference: How the power of diversity creates better groups, firms, schools, and societies. Princeton, NJ: Princeton University Press.

Pajares, M. F. (1992). Teachers' beliefs and educational research: Cleaning up a messy construct. Review of Educational Research, 62(3), 307-332.

Paradis, M. (2008). Language and communication disorders in multilinguals. In B. Stemmer \& H.A. Whitaker (Eds.), Handbook of the neuroscience of language (pp. 341-350). London, UK: Elsevier.

Paris, D. \& Ball, A. F. (2009). Teacher knowledge in culturally and linguistically complex classrooms: Lessons from the golden age and beyond. In L. M. Morrow, R. Rueda, \& D. Lapp (Eds.), Handbook of research on literacy and diversity (pp. 379-395). New York, New York: The Guilford Press.

Payne, R. K. (1996). A framework for understanding poverty. Highlands, TX: aha Process, Inc. 
Pena, M., Maki, A., Kovacic, D. Dehaene-Lambertz, G., Koizumi, H., Bouquet, F., \& Mehler, J. (2003). Sounds and silence: An optical topography study of language recognition at birth. Proceedings of the National Academy of Science, 100(20), 11702-11705.

Perfetti, C. A. \& Frishkoff, G. A. (2008). The neural bases of text and discourse processing. In B. Stemmer \& H.A. Whitaker (Eds.), Handbook of the neuroscience of language (pp. 165-174). London, UK: Elsevier.

Peterson, K. (1984). Methodological problems in teacher evaluation. Journal of Research and Development in Education, 17(4), 62-70.

Petitto, L. A., \& Dunbar, K. (2004, October). New findings from educational neuroscience on bilingual brains, scientific brains, and the educated mind. Session presented at the Building Usable Knowledge in Mind, Brain, \& Education conference. Harvard Graduate School of Education, Cambridge, MA.

Piaget, J. (1959). The language and thought of the child (3rd ed; M Gabain, Trans.). London, UK: Routledge \& Kegan Paul.

Picciano, A. G. (2004). Educational research primer. New York: Continuum.

Pike, K. (1954). Language in relation to a unified theory of the structure of human behavior. Glendale, CA: Summer Institute of Linguistics.

Pinker, S. (2007). The language instinct. New York, NY: First Harper Perennial Modern Classics.

Pulvermuller, F. (2009). Word processing in the brain as revealed by neurophysiological imaging. In M. G. Gaskell (Ed.), The Oxford handbook of psycholinguistics (pp.119-139). Oxford, UK: Oxford University Press. 
Ramos, F. (2009). California/Spain visiting teachers program participants' opinions about the use and effects of students' primary languages in the classroom. International Multilingual Research Journal, 3(2), 51-71.

Rao, S. (2005). Effective multicultural teacher education programs: Methodological and conceptual issues. Education, 126(2), 279-291.

Ravitch, D. (2000). Left back: A century of failed school reforms. New York, NY: Simon $\&$ Schuster.

Redlinger, W. E., \& Park, T. Z. (1980). Language mixing in young bilinguals. Journal of Child Language, 7, 344-352.

Richards, N. (2010). Uttering trees. Cambridge, MA: The MIT Press.

Riccardi, A., Fabbro, F., \& Obler, L. K. (2004). Pragmatically appropriate codeswitching in a quadrilingual with Wernicke's aphasia. Brain and Language, 91, 54-55.

Rizzolatti, G. \& Fabbri-Destro, M. (2010). Mirror neuron mechanism. In G.F. Koob, M. Le Moal, \& R.F. Thompson, (Eds.), Encyclopedia of behavioral neuroscience (pp.240-249). Burlington, MA: Elsevier.

Romaine, S. (2001). Multilingualism. In M. Arnoff \& J. Rees-Miller (Eds.), Handbook of linguistics, (pp. 512-532). Malden, MA: Blackwell.

Romaine, S. (2007). Linguistic diversity and language standardization. In M. Hellinger \& A. Pauwels (Eds.), Handbook of language and communication: Diversity and change (Vol. 9, pp. 685-713). Berlin, Germany: Mouton de Gruyter.

Rossi, E., Denes, G., \& Bastiaanse, R. (2003). A single case study of pathological mixing in a polyglot aphasic. Brain and Language, 87, 46-47. 
Routman, R. (2005). Writing essentials: Raising expectations and results while simplifying teaching. Portsmouth, NH: Heinemann.

Ruiz, R. (1984). Orientation in language planning. Journal of the National Association for Bilingual Education, 8(2), 15-34.

Saffran, J. R. (2003). Statistical language learning: Mechanisms and constraints. Current Directions in Psychological Science, 12(4), 110-114.

Safford, K., \& Kelly, A. (2010). Linguistic capital of trainee teachers: Knowledge worth having? Language and Education, 24(5), 401-414.

Samovar, L.A., Porter, R.E., \& McDaniel, E. R. (2009). Intercultural communication: A reader. Boston, MA: Wadsworth.

Sapir, E. (1921). Language. New York,NY: Harcourt, Brace, \& World.

Saussure, F. de. (1966). Course in general linguistics. New York, NY: McGraw-Hill.

Scharenborg, O., Norris, D., den Bosch, L., \& McQueen, J. M. (2005). How should a speech recognizer work? Cognitive Science, 29, 867-918.

Schoenfeld, A. (1998). Toward a theory of teaching-in-context. Retrieved from http://www.gse.berkeley.edu/faculty/aschoenfeld/TechInContext//teaching-incontext.html

Scribner, S., \& Cole, M. (1981). The psychology of literacy. Cambridge, MA: Harvard University Press.

Scollon, R., \& Scollon, S. W. (2001). Intercultural communication. Malden, MA: Blackwell.

Seliger, H. W., \& Long, M. H. (1983). Classroom oriented research in second language acquisition. Rowley, MA: Newberry House. 
Shadish, W. R., Cook, T. D., \& Campbell, D. T. (2002). Experimental and quasiexperimental designs for casual inference. Boston, MA: Houghton Mifflin.

Shuy, R. W. (1981). Educational linguistics. Neuren Sprachen, 80(5), 455-468.

Skinner, B. F. (1957). Verbal behavior. New York, NY: Appleton-Century-Crofts.

Sleeter, C. (1999). Empowerment through multicultural education: From reproduction to contestation of social inequality through schooling. New York: State University of New York Press.

Slobin, D. I. (1973). Cognitive prerequisites for grammar. In C. Ferguson \& D.L. Slobin (Eds.), Studies in child language development (pp. 175-208). New York, NY: Holt, Rinehart, \& Winston.

Slobin, D. I. (1992). The cross-linguistic study of language acquisition. Hillsdale, NJ: Erlbaum.

Smith, E. (2002). Ebonics: A case history. In L. Delpit \& J. Kilgour Dowdy (Eds.), The skin we speak (pp. 15-30). New York, NY: The New Press.

Smith, F. (1988). Understanding reading. Hillsdale, NJ: Erlbaum .

Snedeker, J., \& Trueswell, J. C. (2004). The developing constraints on parsing decision: The role of lexical-biases and referential scenes in child and adult sentence processing. Cognitive Psychology, 49(3), 283-299.

Snow, C.E. (1972). Mothers' speech to children learning language. Child Development, 43 (2), 549-565.

Sousa, D. A. (2010). What principals need to know about the basics of creating braincompatible classrooms. Bloomington, IN: Solution Tree Press. 
Spolsky, B. (1978). Educational linguistics: An introduction. Roley, MA: Newberry House.

Spolsky, B. (2010). Introduction: What is educational linguistics? In B. Spolsky \& F.M. Hult (Eds.), The handbook of educational linguistics (pp. 1-9). West Sussex, UK: Blackwell Publishing Ltd.

Spolsky, B., \& Shohamy, E. (1999). Language in Israeli society and education. International Journal of Sociology, 137, 93-114.

State Board for Educator Certification (SBEC). (2011). Approved new educator standards. Educator Certification Online System. Retrieved from http://www.sbec.state.tx.us/sbeconline/standtest/edstancertfieldlevl.asp?width=13 $66 \&$ height $=768$

Stemmer, B. (2008). Neuropragmatics: Disorders and neural systems. In B. Stemmer \& H.A. Whitaker (Eds.), Handbook of the neuroscience of language (pp. 175-187). London, UK: Elsevier.

Streeter, L. A. (1976). Language perception in 2-month-old infants show effects of both innate mechanisms and experience. Nature, 259, 39-41.

Strauss, A., \& Corbin, J. (1990). Basics of qualitative research: Grounded theory procedures and techniques. Newbury Park, CA: Sage.

Stubbs, M. (2002). Some basic sociolinguistic concepts. In L.Delpit \& J. Kilgour Dowdy (Eds.), The skin that we speak: Thoughts on language and culture in the classroom (pp. 63-86). New York, NY: New York Press. 
Summak, M .S., Summak, A .E. G., \& Summak, P. S. (2010). Building the connection between mind, brain, and educational practice; roadblocks and some prospects. Procedia Social and Behavioral Sciences, 2, 1644-1647.

Teddlie, C., \& Tashakkori, A. (2009). Foundations of mixed methods research: Integrating quantitative and qualitative approaches in the social and behavioral sciences. Thousand Oaks, CA: Sage.

Tranel, D., Logan, C. G., Frank, R .J., \& Damasio, A .R. (1997). Explaining categoryrelated effects in the retrieval of conceptual and lexical knowledge for concrete entities: operational and analysis of factors. Neuropsychologia, 35, 1329-1340.

Trask, R. L., \& Mayblin, B. (2000). Introducing linguistics. Thriplow, Royston: Icon Books, Ltd.

Triandis, H. C. (2009). Approaches to intercultural communication. In L.A. Samovar, R.E. Porter \& E.R. McDaniel (Eds.), Intercultural Communication (pp. 34-44). Boston, MA: Wadsworth.

Trueswell, J. C., \& Gleitman, L. R. (2009). Learning to parse and its implications for language acquisition. In M. G. Gaskell (Ed.), The Oxford handbook of psycholinguistics (pp.635 - 655). Oxford, UK: Oxford University Press.

Ullman, M. (2008). The role of memory systems in disorders of language. In B. Stemmer \& H.A. Whitaker (Eds.), Handbook of the neuroscience of language (pp. 189198). London, UK: Elsevier.

Underhill, A. (1994). Sound foundations. Oxford, UK: Macmillan.

U.S. Census Bureau. (2010). New Census Bureau report analyzes Nation's linguistic diversity. Retrieved from 
http://www.census.gov/newsroom/releases/archives/american_community_survey $\ldots$

U.S. Department of Education. (2011). International Education Week 2011. Retrieved on November 2, 2011 from http://www.youtube.com/watch?v=gkbmAaLVQJA\&lr=1

Valencia, R. (2011). The plight of Chicano students: An overview of schooling conditions and outcomes. In R. R. Valencia (Ed.), Chicano school failure and success: past, present, and future (pp. 3-41). New York, NY: Routledge.

Van Driel, J.H., Bulte, A.M.W., \& Verloop, N. (2007). The relationship between teachers' general beliefs about teaching and learning and their domain specific curricular beliefs. Learning and Instruction, 17, 156-171.

Vigliocco, G., \& Vinson, D. P. (2009). Semantic representation. In M. G. Gaskell (Ed.), The Oxford handbook of psycholinguistics (pp.195-215). Oxford, UK: Oxford University Press.

Viiri, J., \& Saari, H. (2006). Teacher talk patterns in science lessons: Use in teacher education. Journal of Science Teacher Education, 17, 347-365.

Vygotsky, L .S. (1978). Mind in society: The development of higher psychological processes (M Cole, V. John-Scribner, \& E. Souberman, Eds. \& Trans.). Cambridge, MA: Harvard University Press.

Walenski, M., \& Ullman, M. T. (2005). The science of language. The Linguistic Review, $22,327-346$.

Weinberg, M. (1995). A chance to learn: A history of race and education in the United States. Long Beach, CA: California State University Press. 
Wells, G. (1986). The meaning makers: Children learning language and using language to learn. Portsmouth, NH: Heinemann.

Whorf, B. L. (1956). Language, thought, and reality: Selected writing of Benjamin Lee Whorf. Cambridge, MA: MIT Press.

Wiley, T. G. (2007). Immigrant language minorities in the United States. In M. Hellinger \& A. Pauwels (Eds.), Handbook of language and communication: Diversity and change (Vol. 9, pp. 53-85). Berlin, Germany: Mouton de Gruyter.

Wilson, M. (2001). The changing discourse of language study. English Journal 90(4), 3136.

Wingfield, A., Peelle, J. E., \& Grossman, M. (2003). Speech rate and syntactic complexity as multiplicative factors in speech comprehension by young and older adults. Aging, Neuropsychology, and Cognition, 10, 310-322.

Woods, D. (1997). Decision-making in language learning: A lens for examining learning strategies. The Language Teacher: The Japanese Association for Language Teaching, 21, 67-75.

Woods, D. (2003). The social construction of beliefs in the language classroom. In P. Kalaja \& A. M. F Barcelos (Eds.), Beliefs about SLA: New research approaches (pp. 201-229). Dordrecht. The Netherlands: Kluwer Academic.

Wray, A., \& Bloomer, A. (2006). Projects in linguistics: A practical guide to researching language. London: Hodder Arnold. 
Wren, S. (2002, December). Ten myths of reading instruction. Southwest Educational Development Laboratory, XIV, 3. Retrieved from http://www.sedl.org/pubs/sedlletter/v14n03/2.html

Wynne, J. (2002). We don't talk right. You ask him. In L.Delpit \& J. Kilgour Dowdy (Eds.), The skin that we speak: Thoughts on language and culture in the classroom (pp. 203-219). New York, NY: New York Press.

Yeni-Komshian, G. (1998). Speech perception. In J.B. Gleason \& Nan Bernstein Ratner (Eds.), Psycholinguistics (pp.107-156). Orlando, FL: Harcourt Brace \& Company. 


\title{
APPENDIX A
}

\section{Timeline}

\author{
April $2011 \quad$ Proposal Defense \\ May $2011 \quad$ Human Subjects Approval (Institutional Review Board) \\ Summer $2011 \quad$ Experts will review research instruments \\ Summer $2011 \quad$ Conduct Pilot Study \\ September 2011 Find participants and give them pre-unit attitudes survey and \\ linguistic content assessment \\ October $2011 \quad$ Analyze pre-unit data \\ November $2011 \quad$ Revise and teach unit on linguistics \\ December 2011 Give participants post-unit survey and assessment and \\ questionnaire. \\ December $2011 \quad$ Conduct interviews \\ January 2012 Code and analyze post-unit data and integrate findings \\ February $2012 \quad$ Write final chapters of dissertation \\ March 2012 Defend dissertation
}




\section{APPENDIX B}

\section{Informed Consent}

You are invited to participate in a research study conducted by Kim llosvay from Portland State University, Department of Education. The researcher hopes to learn what beliefs and linguistic content knowledge preservice teachers are bringing into the educator preparation program. The researcher also hopes to learn how a linguistic unit taught to preservice teachers can assist new teachers entering into linguistically diverse classrooms today. The goal of the linguistic unit is to provide knowledge and strategies that will help in effective teaching. This research is being conducted in partial fulfillment of a doctoral degree and is under supervision of advisors at PSU. You were selected as a possible participant in this study because you are a preservice teacher and the cohort has diverse backgrounds that would be significant for the study.

If you decide to participate, you will first be asked to give permission to the researcher to use the surveys, assessments, and questionnaires for research purposes. You will be asked to take a general language survey and linguistic knowledge assessment. As a part of the Multicultural and Urban Education course, the researcher will teach a unit on linguistics. You will then be asked to fill out a post linguistic survey as well as a questionnaire. This will all be done during the courses allotted time so there will be no extra work to be performed outside of class. The only exception to this will be if you are requested to be a participant in an interview session to clarify your answers. You may not receive any direct benefit from this study, but the study may help increase knowledge which will help others in the future. However, there may be direct benefits from the unit of study that can apply to your teaching of linguistically diverse students.

Any information that is obtained in connection with this study and that can be linked to you will be kept confidential. Your participation in this study is voluntary. You do not have to take part in this study and it will not affect your grade whether you chose 
to participate or not. You will be taught the linguistic unit as a part of your course unrelated to your participation. You may also withdraw from this study at any time.

If you have concerns or questions about your participation in this study or your rights as a research subject, please contact the Human Subjects Research Review Committee, Office of Research and Sponsored Projects, 600 Unitus Bldg., Portland State University, (503) 725-4288. If you have questions about the study itself, please contact Kim Ilosvay, (503) 288-4675, Ilosvay@pdx.edu.

Your signature indicates that you have read and understand the above information and agree to take part in the study. Please understand that you may withdraw consent at any time and that by signing, you are not waiving any legal claims, rights, or remedies. The researcher will provide you with a copy of this form for your own records. Please return this form to the researcher after filling it out. Thank you for your consideration in this study. 


\section{APPENDIX C}

\section{Demographics and Language Survey}

Initials

Age
Gender

Country of Origin

Circle one of the answers to the following questions:

1. How often do you travel? Never Occasionally Frequently

2. Do you leave the country? Never Occasionally Frequently

3. Describe language courses you have taken. The courses could include a variety of topics such as linguistics, ESL, language diversity, bilingualism, ...

Which courses were required for degree or licensure?

4. Describe the types of activities/instruction related to acquisition or practice that dealt with language production in the classroom. Possibly present them with a definition.

5. Create a description of aspects of language a teacher should know to help linguistically diverse students in a general education classroom.

6. Were you ever given any specific strategies or techniques to instruct diverse populations? If so, what were they?

7. How would you describe your philosophy on language use and usage in the classroom? Consider issues like "right and wrong" ways to speak, positive and negative views on standard language use, English only schools, ambiguous words, etc.

8. Do you consider yourself monolingual, bilingual, or multilingual? What languages do you speak? How fluent/proficient are you in your native language(s), second language, etc? Are you able to engage in daily conversations with native speakers of the languages? Are you able to use these languages in academic environments? 
(Proficient has been defined as advanced abilities in speaking, reading, and writing by participating in formal and informal exchanges with confidence, accuracy, and clarity; using all necessary tenses in communicative tasks; and to understand and be readily understood by native speakers.) ACTFL \& ASLTA

9. Describe your parent's language abilities. Are they monolingual, multilingual, speak various dialects, proficient in certain languages, etc.?

10. Have you ever been exposed to instruction related to prosody? If yes, please describe the nature of that instruction. (Prosody is the stress, pitch, intonation of a language.)

11. Where do you think your underlying speech accent comes from? Do you feel your present accent was influenced by any other place you lived or people you knew? 


\section{APPENDIX D}

\section{Language Opinion Survey Questions}

Please select the option that most closely relates to your opinion.

\section{Children}

a. are born with the ability to perceive the entire set of possible human sounds and eventually can only hear the sounds they hear around them.

b. do not learn languages as well as adolescents or adults because they do not have the strategies such as mnemonics to remember the vocabulary and grammar rules.

c. who are exposed to more than one language early in life may acquire proficiency in more than one language.

d. fully learn language by repeating words and sounds, without experiencing the objects or events.

2. Classrooms that require a single Standard dialect
A. are merely encouraging the correct way to speak.
B. teach more complex, logical, and expressive ways to speak.
C. better assist student achievement and language competence.
D. deprive speakers and listeners of language resources.

3. Language is primarily a communication system governed by
a. grammatical rules
b. mutual comprehension
c. Neither 
d. Both $a$ and $b$

4. As acquired by each succeeding generation, language
a. becomes more rigid and precise.
b. requires more study to use well.
c. changes slightly.
d. loses outside influences.

5. Language acquisition is
a. acquired - innate
b. learned - explicit
c. a social process, with distinct stages, and limits.
d. genetically wired into every individual, but requires explicit instruction.

6. All of the following are considered language acquisition universals except:

a. There is a finite set of potentially meaning bearing sounds (vowels, consonants, tones) which can be produced by human vocal apparatus.

b. The set of sounds in its entirety is universal and available to all human beings without physical handicap.

c. Language must be carefully taught to children in order for them to acquire a language properly.

d. At some time in adolescence, the ability to acquire language with the same ease as young children atrophies or weakens. 
7. Language is
a. a system of meaning shared by people.
b. a system to organize the world around us.
c. a representation of the culture in which it is used.
d. an expressive system of communication.

8. Black English Dialect
a. carries complex information across a range of cultural groups.
b. conveys much meaning for experienced speakers and listeners.
c. is accepted as a viable communication mode in society today.
d. should be discouraged in public school settings.

9. In the US, it is a teacher's responsibility to

a. teach only in standard English in order to insure that students use the most widely understood language in the United States.

b. teach many dialects and registers of English in order help students negotiate more languages.

c. teach the students version of English to help keep students bonded with their communities.

d. teach the "World Englishes" that exist in the classroom. 
10. A student in your class is taking an oral reading test. He reads "tink" for the word "think." You, as the teacher,

a. mark the word wrong because clearly he has misread the word.

b. ask the student to repeat what he has read to determine if it is a miscue.

c. ask the student what the sentence means. Phonic and graphophonics miscues mean different things.

d. let the student keep reading and ignore the miscue. One word does not matter.

11. If someone spent one hour a day learning a language, how long would it take to become proficient?
a. 1-2 years
b. 3-5 years
c. $6-9$ years
d. You can't learn a language in an hour a day.

12. It is necessary to know about English-speaking cultures in order to speak and understand English.
a. True
b. False

13. As a teacher, it is important to speak English
a. using academic English with perfect pronunciation.
b. using the local dialect of English pronunciation.
c. correctly or not teach students directly. 
d. with varying pronunciations to allow students experiences in World Englishes.

14. During speaking, teachers should pay the most attention to their
a. pronunciation.
b. grammar.
c. vocabulary.
d. A, b, \& c are equally important.

15. English is a
a. very difficult language to understand and speak.
b. language of medium difficulty to understand and speak.
c. easy to understand and speak.
d. different for everyone.

16. It is easier to read and write English than to learn how to speak or understand it.
a. True
b. False

17. People who speak many languages
a. are intelligent.
b. should focus on one.
c. get confused easily.
d. can only truly be proficient in one of the languages.

18. The most important aspect of comprehending a speaker is to know
a. the vocabulary.
b. the syntax. 
c. the accent.

d. not just one of these things.

19. When two people engage in the negotiation of language,

a. they gain better comprehension of the language.

b. they acquire the language.

c. they experience frustration that interferes with language usage.

d. they become aware of individual style differences of language.

20. Students learning English

a. experience a complex process that is fundamentally the same for each person.

b. undergo various processes of language acquisition depending on their native language(s).

c. find it easier to learn if they are native speakers of a language that is linguistically similar; for example Spanish.

d. can all use the same language program to learn English regardless of other languages spoken.

21. In the US, who is primary responsibility of ensuring appropriate instruction of English language learners?
a. General classroom teachers
b. ESL teachers
c. Language Arts teachers
d. all teachers are equally responsible 
22. Our memory for language is best when
a. language learning is connected to languages already known.
b. associations are made with the environment while learning.
c. vocabulary is repeated constantly.
d. language is practiced in context.

23. Children that speak several languages
a. usually live in poverty
b. usually come from highly educated families
c. will take longer to become proficient in English due to the lack of support at home
d. usually have a specific genetic make-up and natural ability

24. Monolingual/monocultural policies in schools,
a. create equality among students.
b. benefit students by teaching language and culture of power.
c. create student failure by exclusion of multiple perspectives.
d. divide students further due to lack of acknowledgment of difference.

25. A teacher's primary instructional focus should be
a. supporting of multilingual students.
b. teaching curriculum at the level of the benchmark students. 
c. ensuring the comprehension of all students.

d. an approach that challenges higher achieving students and reviews for struggling students.

26. According to neuroscientists, the 3 most important ingredients the brain needs for optimal language learning are

a. relevance, emotion, and context.

b. context, repetition, and stimulus.

c. patterns, explanation, and time.

d. visual, kinesthetic, and oral.

Select the agreement level that best describes your beliefs of each question.

27. Academic success will fall if teachers allow Black English to be spoken in school.

\begin{tabular}{|l|l|l|l|l|}
\hline Strongly agree & Agree & $\begin{array}{l}\text { Neither agree } \\
\text { or disagree }\end{array}$ & Disagree & $\begin{array}{l}\text { Strongly } \\
\text { disagree }\end{array}$ \\
\hline
\end{tabular}

28. Speaking Black English limits a student's comprehension of other languages.

\begin{tabular}{|l|l|l|l|l|}
\hline Strongly agree & Agree & $\begin{array}{l}\text { Neither agree } \\
\text { or disagree }\end{array}$ & Disagree & $\begin{array}{l}\text { Strongly } \\
\text { disagree }\end{array}$ \\
\hline
\end{tabular}

29. Students remember more information when they use a process that involves implicit (hands-on and unconscious learning) and explicit (short term and working memory) learning.

\begin{tabular}{|l|l|l|l|l|}
\hline Strongly agree & Agree & $\begin{array}{l}\text { Neither agree } \\
\text { or disagree }\end{array}$ & Disagree & $\begin{array}{l}\text { Strongly } \\
\text { disagree }\end{array}$ \\
\hline
\end{tabular}


30. The elimination of nonstandard dialects of English creates social stability.

\begin{tabular}{|l|l|l|l|l|}
\hline Strongly agree & Agree & $\begin{array}{l}\text { Neither agree } \\
\text { or disagree }\end{array}$ & Disagree & $\begin{array}{l}\text { Strongly } \\
\text { disagree }\end{array}$ \\
\hline
\end{tabular}

31. As a part of the Chinese culture, Mandarin dialects should be encouraged in the classroom.

\begin{tabular}{|l|l|l|l|l|}
\hline Strongly agree & Agree & $\begin{array}{l}\text { Neither agree } \\
\text { or disagree }\end{array}$ & Disagree & $\begin{array}{l}\text { Strongly } \\
\text { disagree }\end{array}$ \\
\hline
\end{tabular}

32. In a school that has primarily native Spanish speakers, Spanish should be taught as well as English.

\begin{tabular}{|l|l|l|l|l|}
\hline Strongly agree & Agree & $\begin{array}{l}\text { Neither agree } \\
\text { or disagree }\end{array}$ & Disagree & $\begin{array}{l}\text { Strongly } \\
\text { disagree }\end{array}$ \\
\hline
\end{tabular}

33. Society does not benefit from usage of nonstandard dialects of English.

\begin{tabular}{|l|l|l|l|l|}
\hline Strongly agree & Agree & $\begin{array}{l}\text { Neither agree } \\
\text { or disagree }\end{array}$ & Disagree & $\begin{array}{l}\text { Strongly } \\
\text { disagree }\end{array}$ \\
\hline
\end{tabular}

34. The human brain processes language by creating different pathways to memory in different areas of the brain.

\begin{tabular}{|l|l|l|l|l|}
\hline Strongly agree & Agree & $\begin{array}{l}\text { Neither agree } \\
\text { or disagree }\end{array}$ & $\begin{array}{l}\text { Disagree } \\
\text { disagree }\end{array}$ \\
\hline
\end{tabular}

35. A teacher should correct a student's use of nonstandard English.

\begin{tabular}{|l|l|l|l|l|}
\hline Strongly agree & Agree & $\begin{array}{l}\text { Neither agree } \\
\text { or disagree }\end{array}$ & Disagree & $\begin{array}{l}\text { Strongly } \\
\text { disagree }\end{array}$ \\
\hline
\end{tabular}




\section{APPENDIX E \\ Linguistic Content Assessment}

Please answer the following questions with the best choice present.

1. To develop language skills, students must have knowledge of the cognitive elements such as
a. the sounds, meanings, and grammar of language.
b. culture and sounds of the language.
c. stresses on words and sentences.
d. knowing the correct terminology for talking about language.

2. Deficits in the ability to process and produce language are due predominantly to
a. lack of word pronunciation and memory skills.
b. lack of writing skills.
c. lack of identifying sounds in words.
d. lack of spelling-sound rules.

3. Teachers and students switch languages to
a. clarify and elaborate information.
b. mark their identity.
c. show power. 
d. All of the above.

4. Effective intercultural communication

a. Requires sensitivities to the social interactional aspects of language.

b. Uses knowledge of culture and language.

c. Both $a$ and $b$.

d. Neither $a$ and $b$.

5. An example of a morpheme, the smallest units of sound with meaning, is
a. The /s/ in cats.
b. Cat, the whole word.
c. Both a and b.
d. Neither a nor b.

6. The hardest part of understanding continuous speech is
a. picking out the grammar.
b. hearing word boundaries.
c. understanding the accent.
d. deciding what is meaningful. 
7. The only difference between the phoneme $/ \mathrm{k} /$ and $/ \mathrm{d} /$ is:

a. Place of articulation - the location in the mouth of the speech sound

b. Manner of articulation - how the tongue, lips, and jaw are involved in making a speech sound

c. Aspiration - the act of breathing during a speech sound

d. Both a and b

8. Language change most often occurs at:

a. a phonetic level - individual speech sounds

b. a morphological level - word structure

c. a syntactic level - grammatical

d. a semantic level - literal meaning

9. Considering pronunciation, not orthography (writing), circle how many syllables the following words have:
a. Lightening $2 \quad 3 \quad 4$
b. Spoil $\quad 1 \quad 2 \quad 3$
$\begin{array}{llll}\text { c. Walked } & 1 & 2 & 3\end{array}$ 

d. Decidedly
3
4
5

10. How many phonemes (speech sound) in the word edge?
a. 1
b. 2
c. 3
d. 4

11. What is the third speech sound in wretch?
a. /ch/
b. /e/
c. /t/

12. Do hut and foot rhyme in your speech?
a. Always/ usually rhyme
b. Sometimes rhyme
c. Never/very rarely rhyme

13. Do these words rhyme? Yes or No
a. Merry Murray
Yes
No
b. Spider Writer
Yes
No
c. Sock Talk
Yes
No 

d. Water Hotter
Yes
No

14. For the following words, find a word in the row that ends with the same sound:
a. Dogs: his ducks piece
b. Knew: sew coy igloo
c. Shrink: thing antique fatigue

15. Where in the mouth is the sound $/ \theta /-/ \mathrm{th} /$ in think?
a. Front of the mouth (interdental)
b. Back of the mouth (velar)
c. Throat (glottal)

16. Comprehension of language relies on
a. the use of standard language.
b. word and sentence level meaning, and concept recognition.
c. using the technique, "repeat after me."
d. knowledge of the culture surrounding the language.

17. A teacher asks a student to fill in the blank to the sentence, "Mary fell off the ." A student replies "brain”.
a. The student is displaying meaning and grammar knowledge.
b. The teacher should teach the student more vocabulary. 
c. The student is displaying grammar, but not meaning knowledge.

d. The student is not displaying any knowledge of language systems.

18. The key to learning language is to create synaptic connections by
a. experiencing language
b. repetition
c. getting the 'right' answer
d. stimulating the left hemisphere

19. Speaking one dialect
a. limits understanding of other dialects
b. allows students to speak to more people
c. means that students may not hear sounds from another dialect
d. enhances student performance in language activities

20. I asked Alvin if he knew how to play basketball and I axt Alvin does he know how to play basketball are examples
a. of correct and incorrect English dialects
b. of different ways to say the same thing
c. of the influence of inappropriate instruction
d. of bilingualism

21. Knowledge of auditory, visual, and physiological aspects of sound are useful
a. when a learner produces a sound that is not standard English.
b. to language teachers only.
c. when students are learning a new language.
d. when a student uses the wrong vocabulary word. 
22. It is impossible to understand a teacher's speech without
a. listening to and comprehending the intonation.
b. hearing word and speech boundaries.
c. proficiency in the teacher's language.
d. knowing the sounds/phonemes of that language.

23. Language use and comprehension are dependent on which three aspects:
a. Vocabulary, context, rhyming
b. Speaker, listener, context
c. Expression, grammar, volume
d. Visuals, context, references

24. Black English lacks basic linguistic concepts like plurality and negation.

\begin{tabular}{|l|l|l|l|l|}
\hline Strongly agree & Agree & $\begin{array}{l}\text { Neither agree } \\
\text { or disagree }\end{array}$ & Disagree & $\begin{array}{l}\text { Strongly } \\
\text { disagree }\end{array}$ \\
\hline
\end{tabular}

25. The best type of instructional environment for diverse populations is
a. explicit instructions and quiet environment
b. flexible instructions and environment
c. structured and quiet
d. structured with frequent student discussion 
Linguistic Diversity 


\section{APPENDIX F}

Interview Protocols

During this interview, the researcher will provide definitions and explanatory examples to support the structure of the following questions. The interview is to clarify answers that the participants provided in the survey and assessment.

1. What aspects of your language experience do you feel will be a benefit to you entering a culturally and linguistically diverse classroom?

2. How do you feel your classroom experience with language will benefit you in the classroom? (What and how specifically?)

3. How would you describe your culture? How do you think it affects your language?

4. What counts as language competence?

5. What are potential language issues that you foresee encountering in the classroom?

6. What are some strategies/techniques can you use to bridge comprehension misunderstandings?

7. How will you know if a misunderstanding is based on vocabulary comprehension, phonological gaps, or a separate issue?

8. What do you believe influences your language behavior? How? Why?

9. How would you characterize your communication style?

10. Do your characteristics if speech change based on the context you are speaking in? How?

How would you respond to the following statements?

a. English should be the only language spoken during school-sponsored activities.

b. There is no point in trying to communicate with students/parents who speak a different language.

c. The more bilingual children there are in a classroom, the more need for special education services.

d. Children who speak a native language other than English are the primary responsibility of ESL teachers.

11. Can you explain...? 
12. Will you tell me more about...?

13. Suppose... What would that be like?

14. Would you like to add anything before we end this interview? 


\title{
APPENDIX G
}

\section{Permission}

Kimberly llosvay

$\operatorname{Jan} 21$

\section{to lyndsey.nickels}

\begin{abstract}
Hello!
I am writing to request permission for the use of your language processing model. I am finishing my dissertation work at Portland State University and would like to use the model in my dissertation. I am looking at preservice teachers knowledge of language processing and language acquisition. The use of this model would strengthen the arguments that I am making about the types of foundational language knowledge that educators need to enter into diverse classrooms. If I can provide you with further information, please let me know. Also, if I can have permission to use your language processing model, I would appreciate it. Thank you,

Kim llosvay
\end{abstract}

Lyndsey Nickels lyndsey.nickels@mq.edu.au

Mar 14

to me

Dear Kim, No you didn't rush me. I guess your request just confused me a little! I assume that you downloaded the model from my website? Anyhow, to my mind once something is in the public domain, people are free to use it as they wish - as long as they give suitable acknowledgement/citation. (In otherwords, yes I'm happy for you to use my model!)

Your approach seems very sensible indeed, good luck with your dissertation, Best wishes, Lyndsey

Keating, Patricia keating@humnet.ucla.edu $\operatorname{Jan} 30$

to me

Dear Ms. llosvay,

Permission to reproduce the IPA chart in your dissertation "A QUALITATIVE STUDY OF LANGUAGE BELIEFS AND LINGUISTIC KNOWLEDGE IN PRESERVICE TEACHERS USING INTEGRATED FRAMEWORKS" is granted as long as acknowledgment is made to the IPA. Acknowledgment can be made as follows:

"Reprinted with permission from The International Phonetic Association. Copyright 2005 by International Phonetic Association."

We'd also appreciate it if you could note how to contact the IPA (e.g. the IPA web site http://www.langsci.ucl.ac.uk/ipa/.).

A downloadable IPA chart can be found here: http://www.langsci.ucl.ac.uk/ipa/IPA chart \%28C\%292005.pdf or here:

http://web.uvic.ca/ling/resources/ipa/charts.htm 
Linguistic Diversity 256

Patricia Keating

Professor of Linguistics, UCLA 310-794-6316/ keating@humnet.ucla.edu

UCLA personal website: http://www.linguistics.ucla.edu/people/keating/keating.htm

Phonetics Lab website: http://www.linguistics.ucla.edu/faciliti/uclaplab.html 